\title{
Potent hydrazide-based HDAC inhibitor with superior pharmacokinetic profile for efficient treatment of acute myeloid leukemia in vivo
}

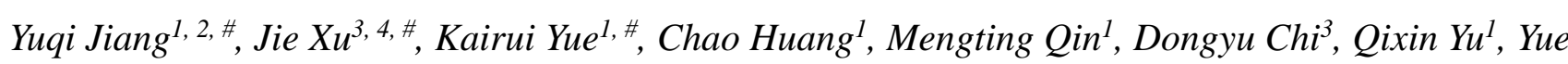
Zhu ${ }^{3}$, Xiaohan Hou ${ }^{1}$, Tongqiang $\mathrm{Xu}^{1}$, Min $\mathrm{Li}^{4}$, C. James Chou ${ }^{5,}$, and Xiaoyang $\mathrm{Li}^{1,2,}$ *

${ }^{1}$ Key Laboratory of Marine Drugs, Chinese Ministry of Education, School of Medicine and Pharmacy, Ocean University of China, Qingdao 266071, China;

${ }^{2}$ Laboratory for Marine Drugs and Bioproducts, Qingdao National Laboratory for Marine Science and Technology, Qingdao 266003, China;

${ }^{3}$ Oncology and Immunology Unit, Research Service Division, WuXi AppTec, Nantong 226299, China; ${ }^{4}$ School of Computer Science and Engineering, Central South University, Changsha 410083, China; ${ }^{5}$ Department of Drug Discovery and Biomedical Sciences, College of Pharmacy, Medical University of South Carolina, Charleston, South Carolina 29425, United States.

\section{Corresponding Authors}

Xiaoyang Li: lixiaoyang@ouc.edu.cn; C. James Chou: chouc@musc.edu. 


\section{Table of Contents}

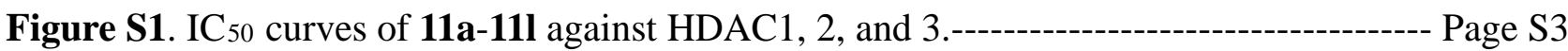

Figure S2. Antiproliferative curves of $\mathbf{1 1 a - 1 1 l}$ to MV4-11 cell line.----------------------------- Page S3

Figure S3. Michael reaction of compound 18 in the presence of 2-mercaptoethanol.---------- Page S3

Figure S4. HE staining of lung, kidney and spleen for vehicle and $\mathbf{1 1 h}(4 \mathrm{mg} / \mathrm{kg})$ treated group

Page S4

Figure S5. Body weight change of tumor-bearing mice after treatment of $11 \mathrm{~h}$ and Panobinostat.-----Page S5

Figure S6. Potential binding mode of 13a with catalytic site of HDAC1, HDAC8, HDAC6, HDAC7 in silico. -Page S5

${ }^{1} \mathrm{~N}$ NMR and ${ }^{13} \mathrm{C}$ NMR Spectrums for all the target compounds. page S7-S27

HPLC traces and purity of all the target compounds -page S28-S33 

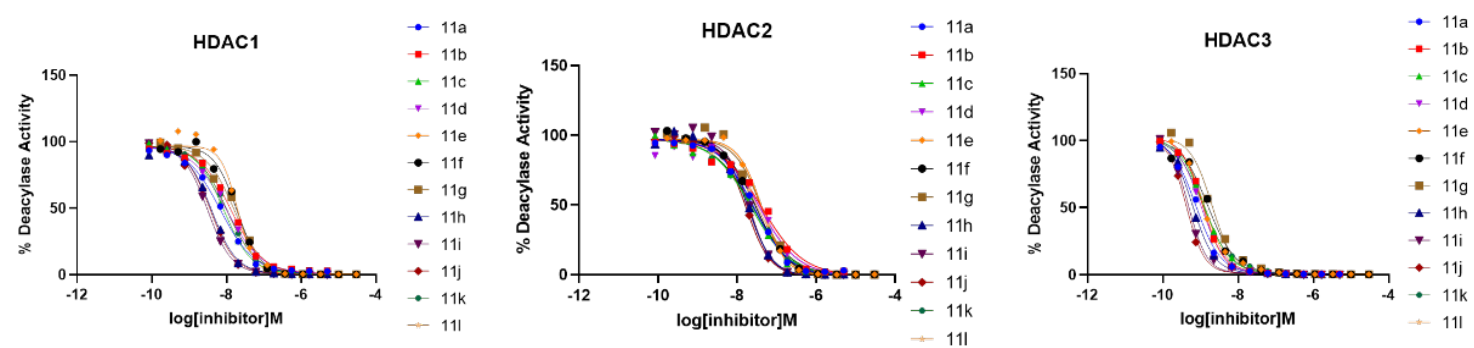

Figure S1. IC 50 curves of 11a-11l against HDAC1, 2, and 3.

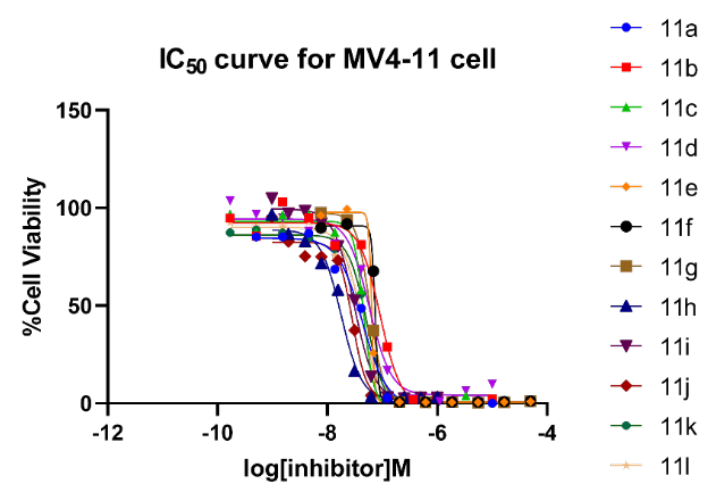

Figure S2. Antiproliferative curves of 11a-11l to MV4-11 cell line.

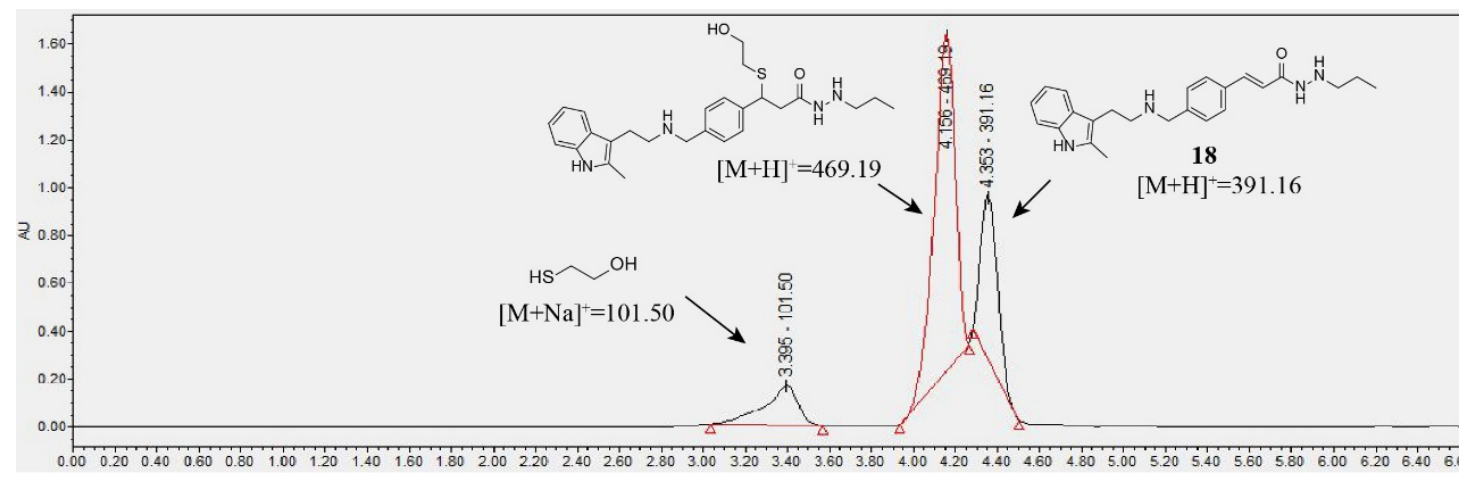

Figure S3. Michael reaction of compound 18 in the presence of 2-mercaptoethanol. 18 was subjected with 2-mercaptoethanol for $12 \mathrm{~h}$, then the components of the reaction mixtures were detected by UV detection of ESI LCMS, performed on a Waters e2695 HPLC instrument using a XBridge C18 column $(5 \mu \mathrm{m}, 4.6 \mathrm{~mm} \times 150 \mathrm{~mm})$ with a gradient of water/acetonitrile plus $0.1 \%$ formic acid $(0-8 \mathrm{~min}$ from $10-100 \%$ acetonitrile, $8-10$ min from $100 \%$ to $10 \%$ acetonitrile). Compound $\mathbf{1 8}$ can react with 2mercaptoethanol within $12 \mathrm{~h}$. 
Representative images for HE staining of Lung

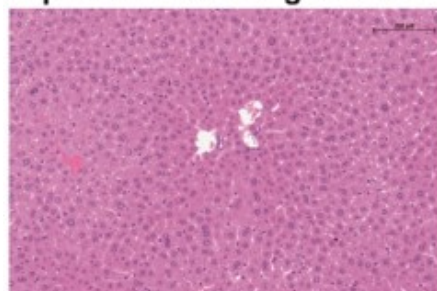

Vehicle-1

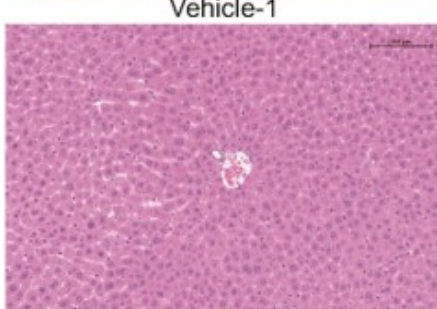

$11 \mathrm{~h}-14 \mathrm{mg} / \mathrm{kg}$

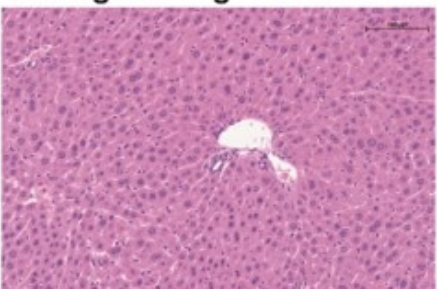

Vehicle-2

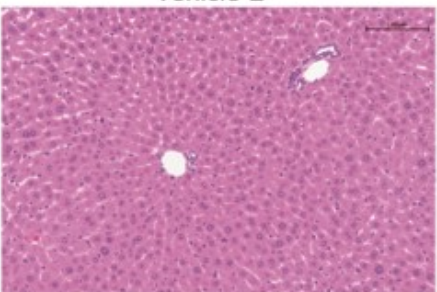

$11 \mathrm{~h}-24 \mathrm{mg} / \mathrm{kg}$

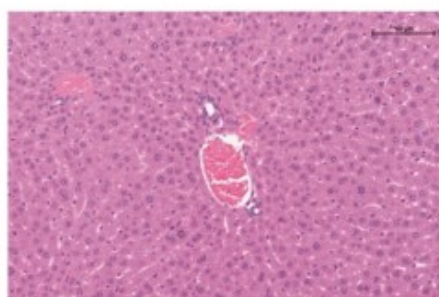

Vehicle-3

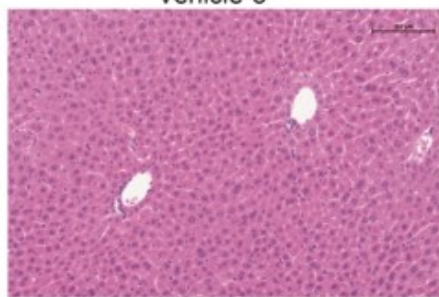

$11 \mathrm{~h}-34 \mathrm{mg} / \mathrm{kg}$

\section{Representative images for HE staining of Kidney}

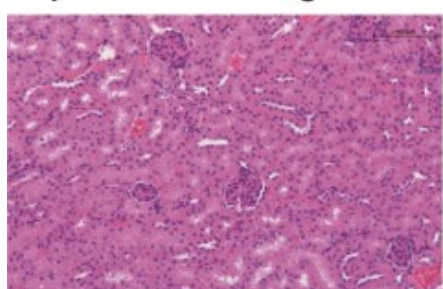

Vehicle-1

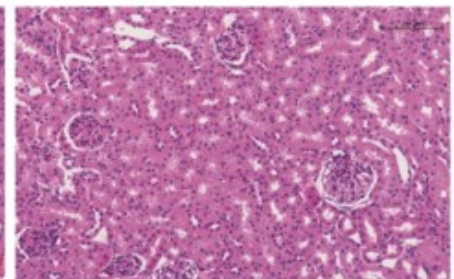

Vehicle-2
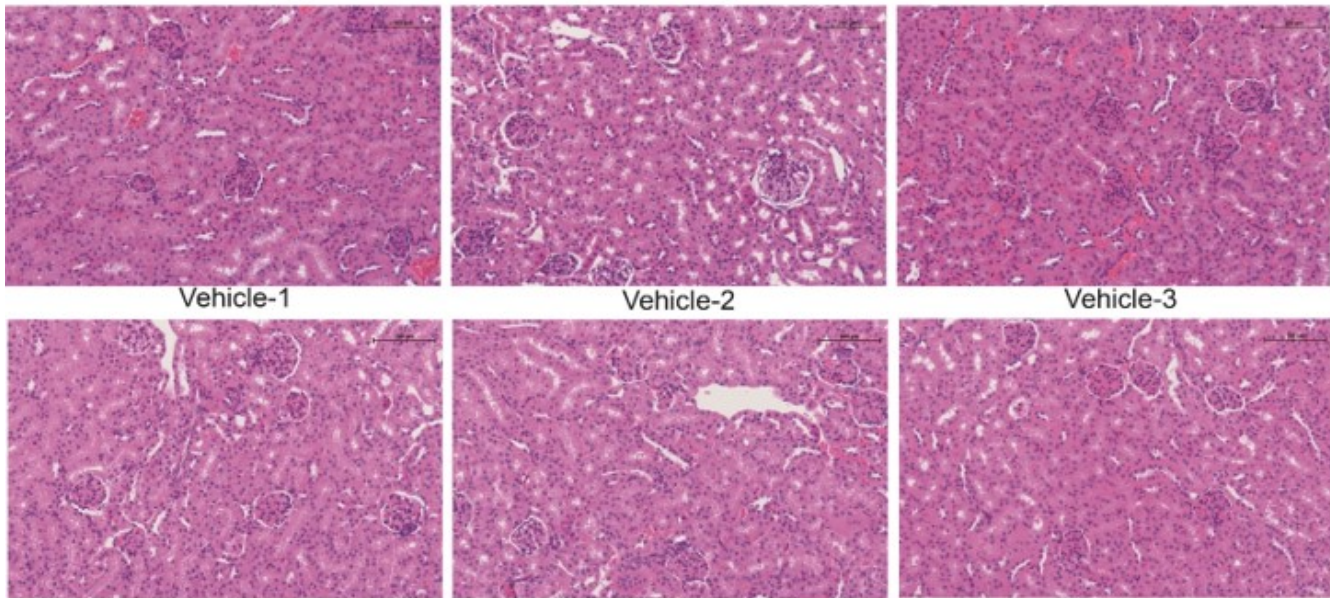

$11 \mathrm{~h}-14 \mathrm{mg} / \mathrm{kg}$

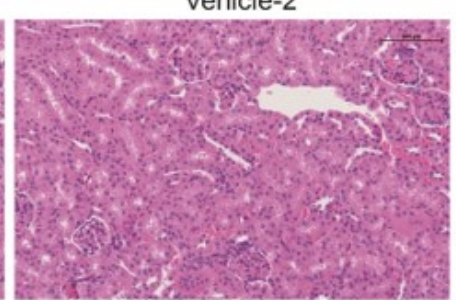

$11 \mathrm{~h}-24 \mathrm{mg} / \mathrm{kg}$

Representative images for HE staining of Spleen

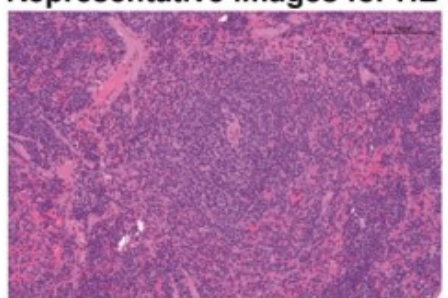

Vehicle-1
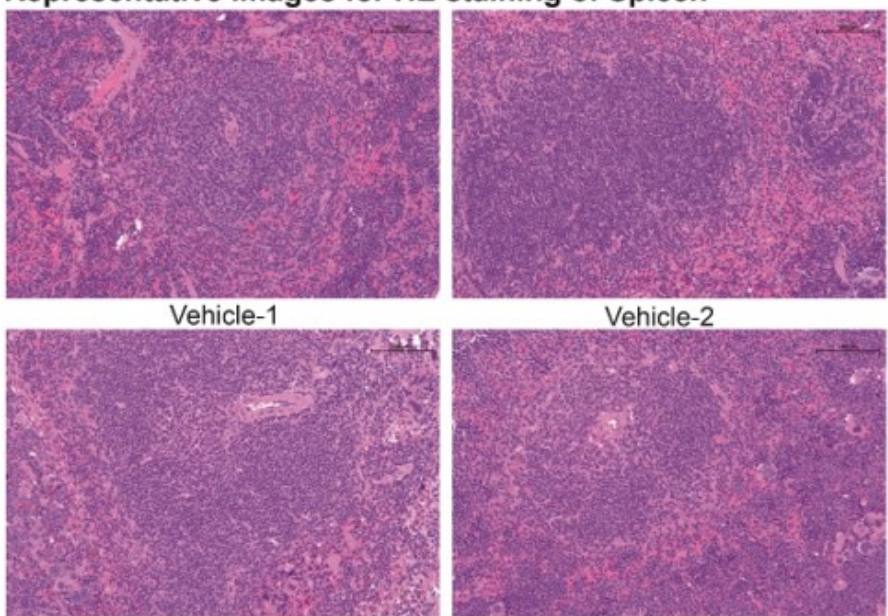

$11 \mathrm{~h}-14 \mathrm{mg} / \mathrm{kg}$

Vehicle-2

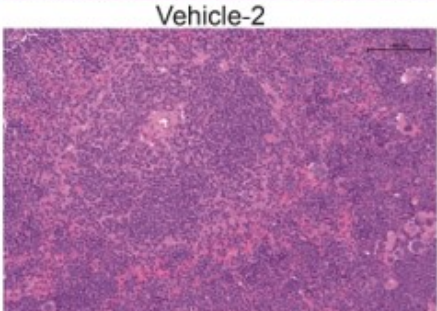

$11 \mathrm{~h}-24 \mathrm{mg} / \mathrm{kg}$

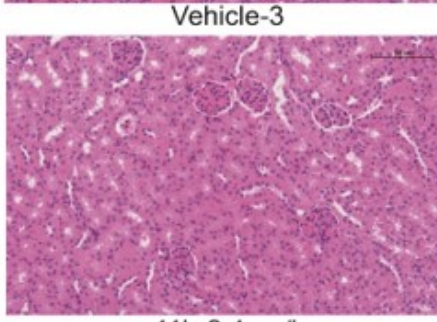

$11 \mathrm{~h}-34 \mathrm{mg} / \mathrm{kg}$

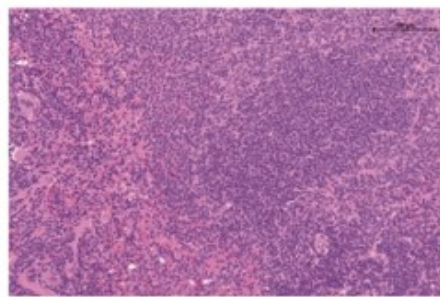

Vehicle-3

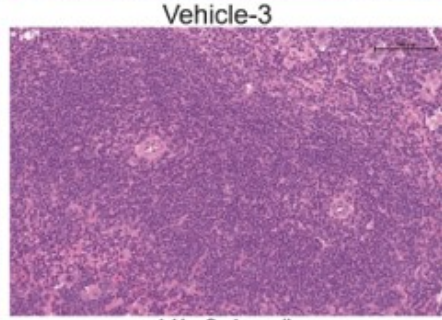

$11 \mathrm{~h}-34 \mathrm{mg} / \mathrm{kg}$

Figure S4. HE staining of lung, kidney and spleen for vehicle and $\mathbf{1 1 h}(4 \mathrm{mg} / \mathrm{kg})$ treated group. 


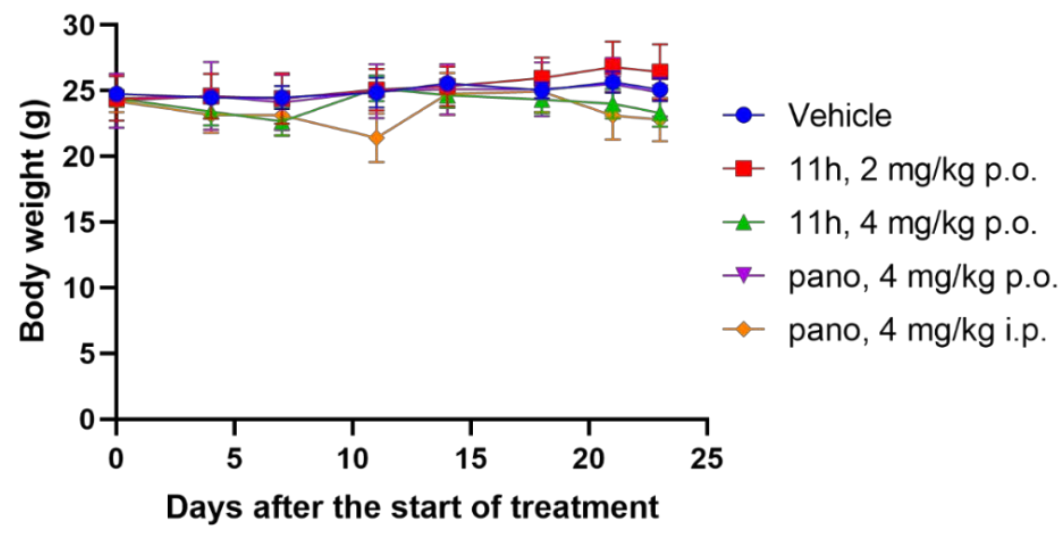

Figure S5. Body weight change of tumor-bearing mice after treatment of $\mathbf{1 1 h}$ and Panobinostat.
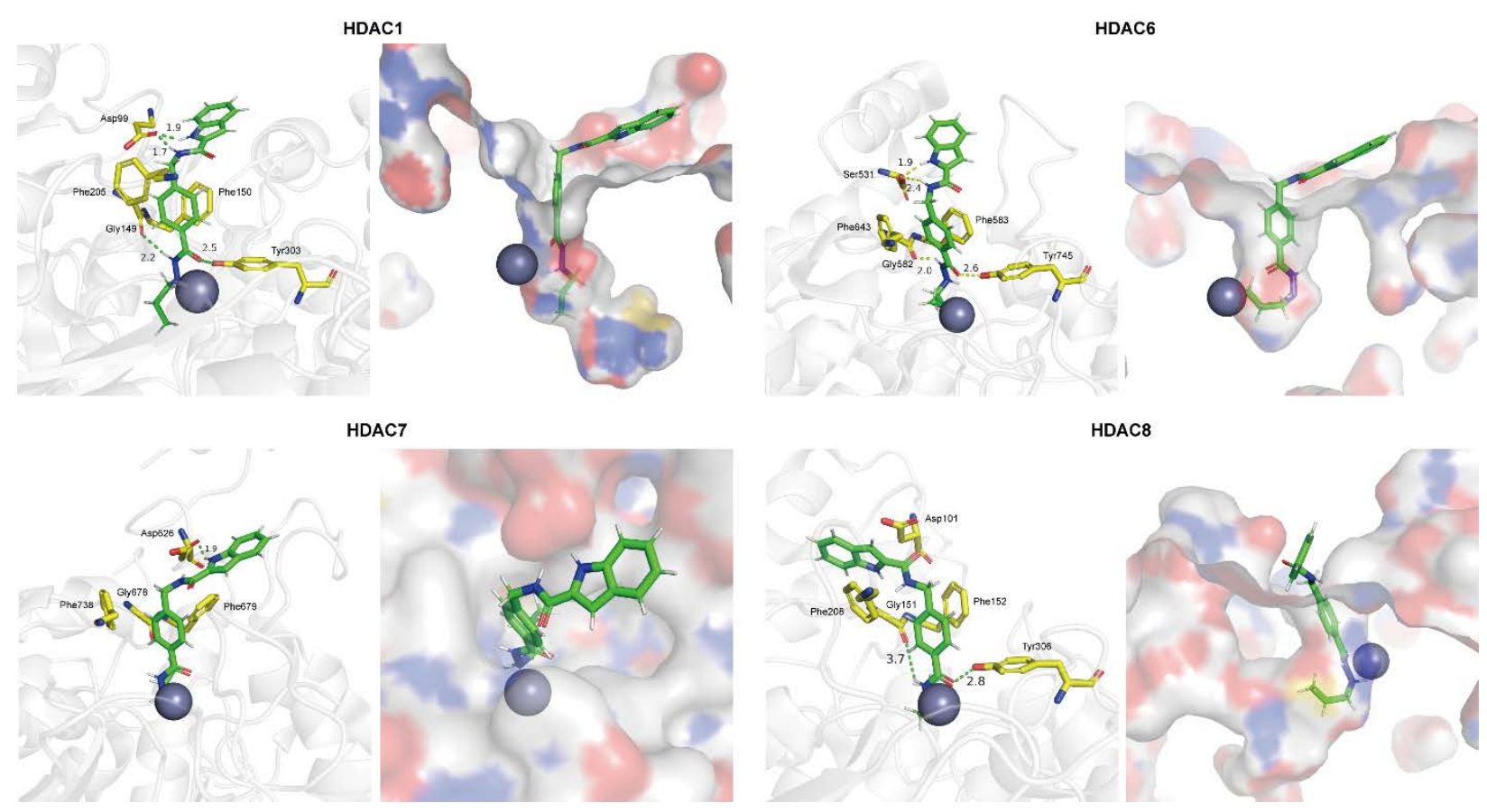

Figure S6. Potential binding mode of 13a with catalytic site of HDAC1, HDAC8, HDAC6, HDAC7 in silico. To elucidate the different binding models of compound $\mathbf{1 1 h}$ with class I, IIa, IIb HDACs, we conducted molecular docking studies with available human crystal structures of HDAC1, 6, 7 and 8 (PDB ID: 4BKX, 5WGL, 3ZNR and 6HSK, respectively). For HDAC1, compound 11h fits perfectly with the catalytic pocket. In detail, the carbonyl group of Asp99 on the protein surface forms hydrogen bonds with NH of indole and amide group in 11h's cap region. The benzyl of the linker group can form $\pi-\pi$ stacking interaction with Phe205 and Phe105 in the binding cavity. The carbonyl group and NH of 
the hydrazide chelate with zinc ion in a bidentate manner. In addition, carbonyl group and NH of hydrazide can form hydrogen bond with Try303 and Gly149, respectively. The propyl tail of $\mathbf{1 1 h}$ is unable to stretch at the pocket bottom of HDAC6, which affects the interaction between hydrazide and zinc ion. This might be the reason for the poor activity of $\mathbf{1 1 h}$ against HDAC6. $\mathbf{1 1 h}$ cannot completely occupy the binding cavity of HDAC7 as the cavity is much wider than the other HDAC subtypes, thus weakening the interaction. On the other hand, the lack of hydrogen bond between hydrazide (NH and carbanyl group) of $\mathbf{1 1 h}$ and residues of HDAC7 makes the binding of $\mathbf{1 1 h}$ to HDAC7 weaker than that to HDAC1. 11h displays similar binding modes with HDAC1 and HDAC8, however, the surface of HDAC8 cannot form hydrogen bonds with the cap group of $\mathbf{1 1 h}$, which might be the reason of the lower activity of $\mathbf{1 1 h}$ against HDAC8. 
${ }^{1} \mathrm{H}$ NMR and ${ }^{13} \mathrm{C}$ NMR of all the products
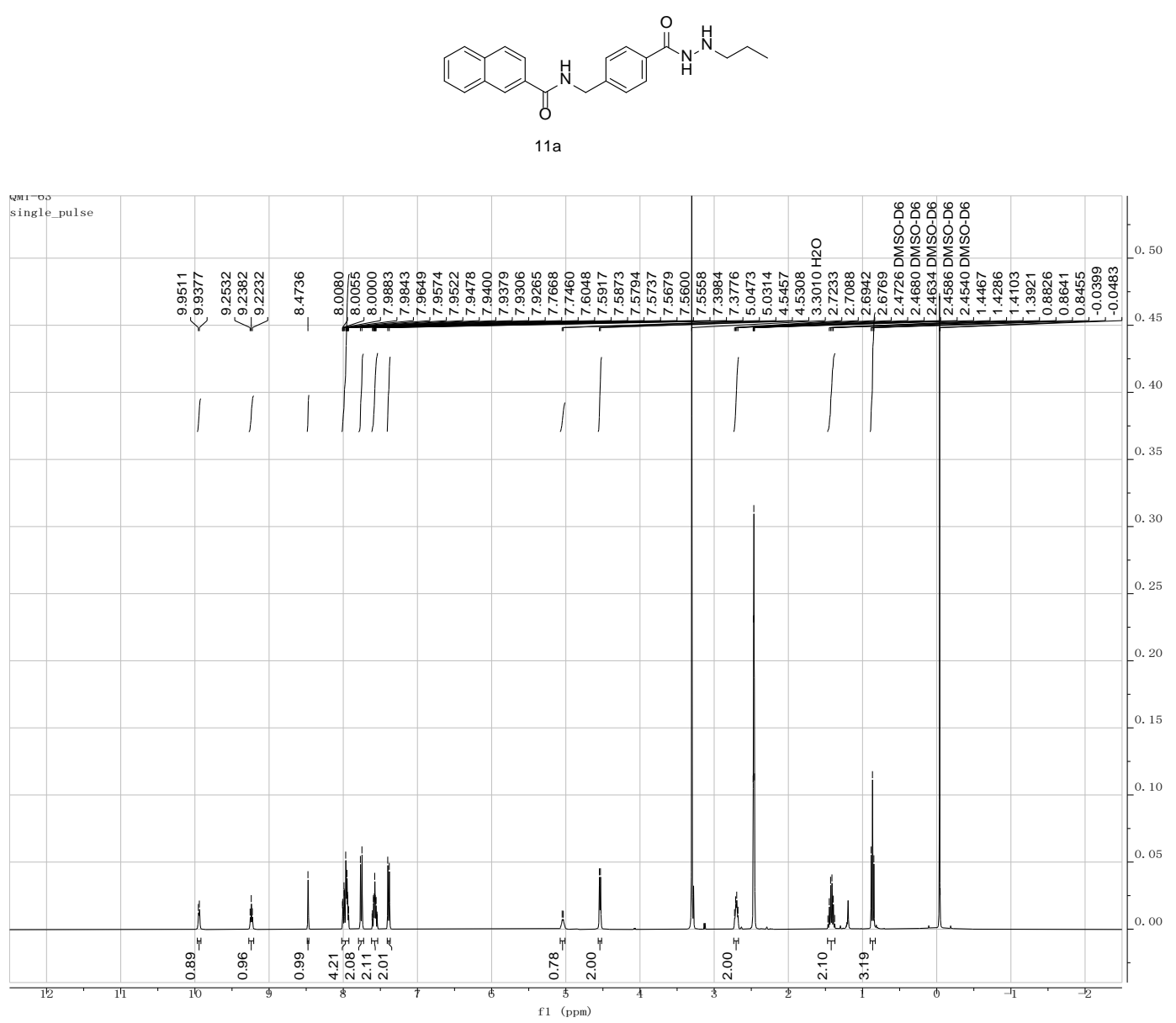

${ }^{1} \mathrm{H}-\mathrm{NMR}$ of 11a

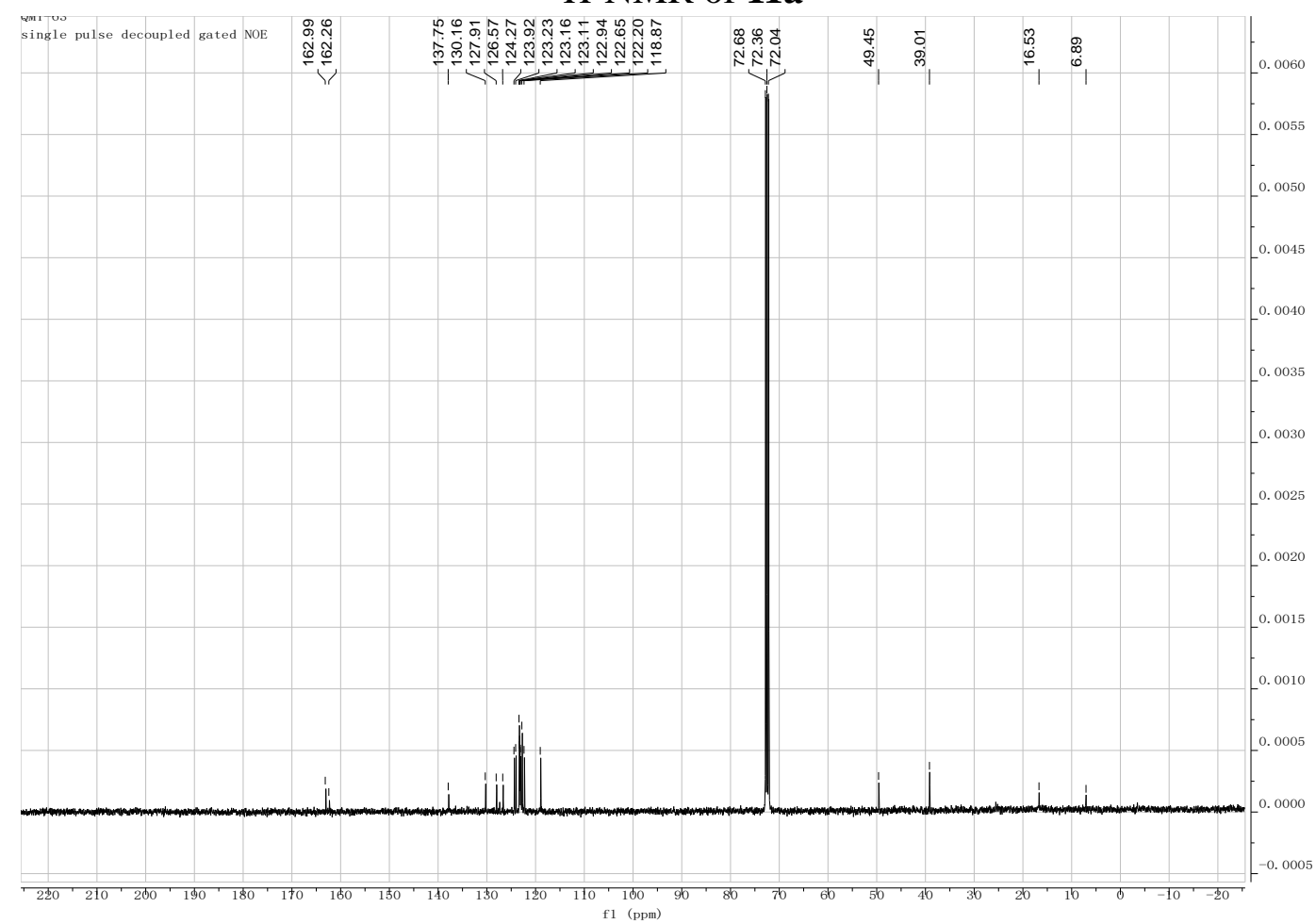

${ }^{13} \mathrm{C}-\mathrm{NMR}$ of 11a 


$$
\text { (11b }
$$

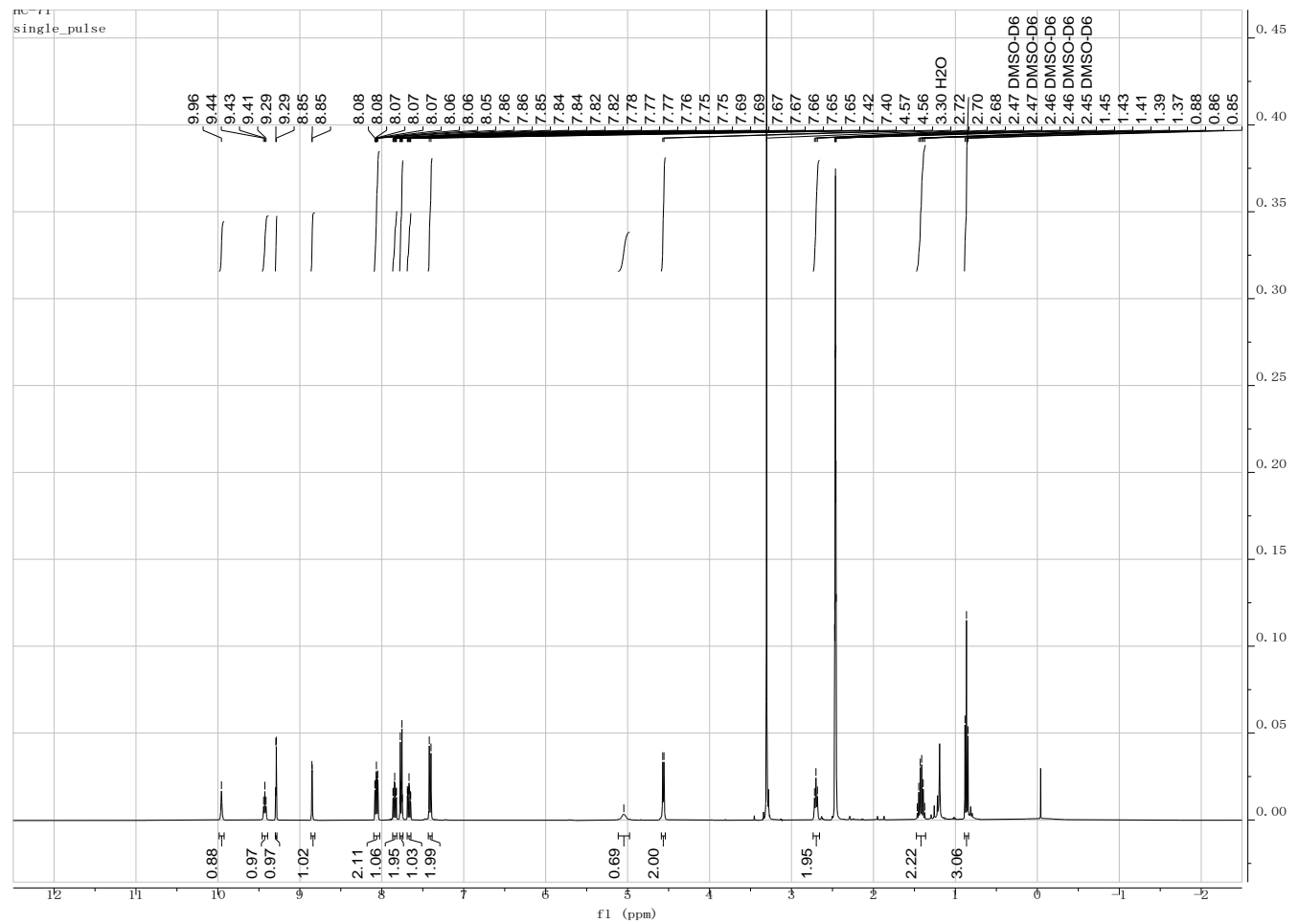

${ }^{1} \mathrm{H}-\mathrm{NMR}$ of $\mathbf{1 1 b}$

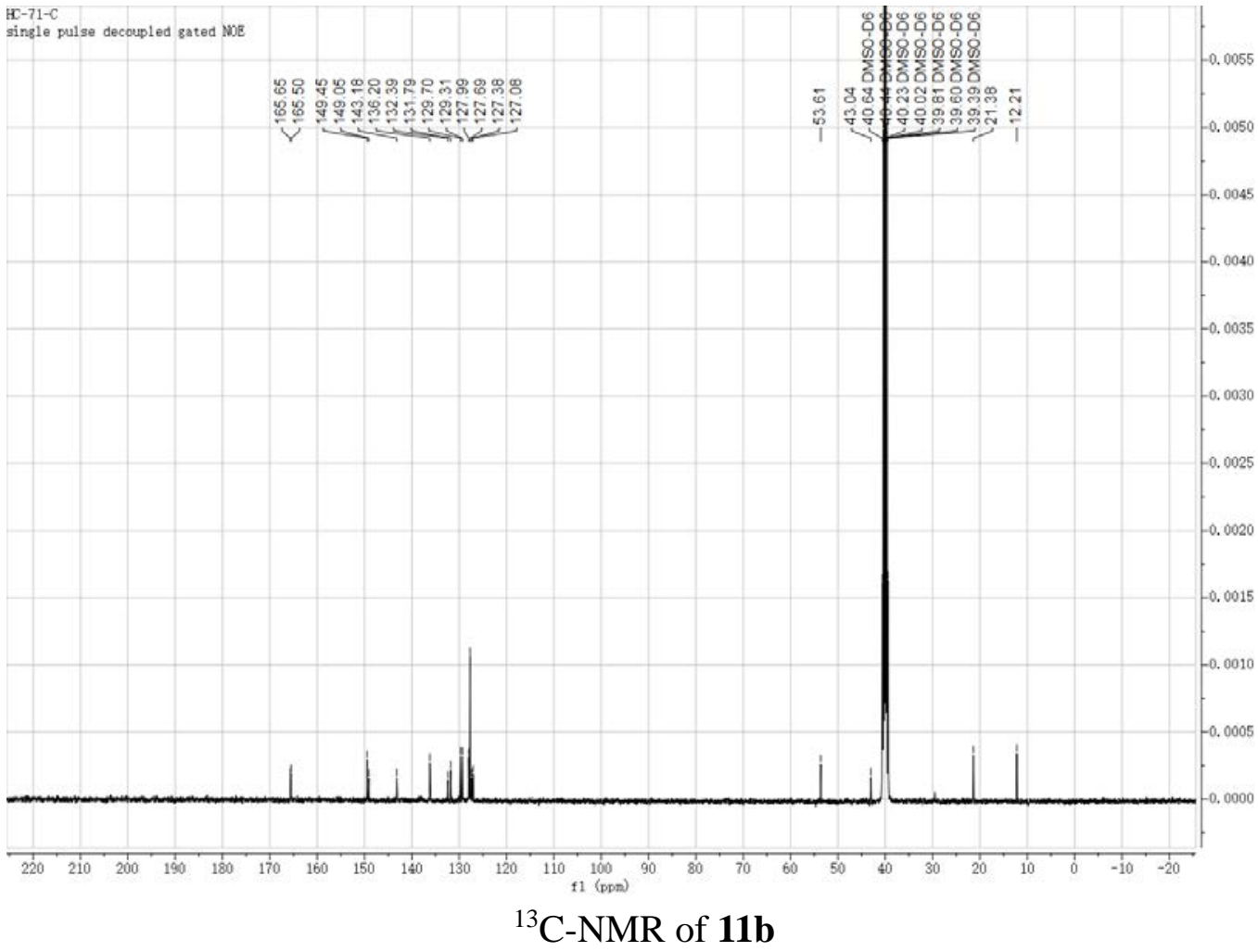


<smiles>CCCNNC(=O)c1ccc(CNC(=O)c2cc3ccccc3cn2)cc1</smiles>

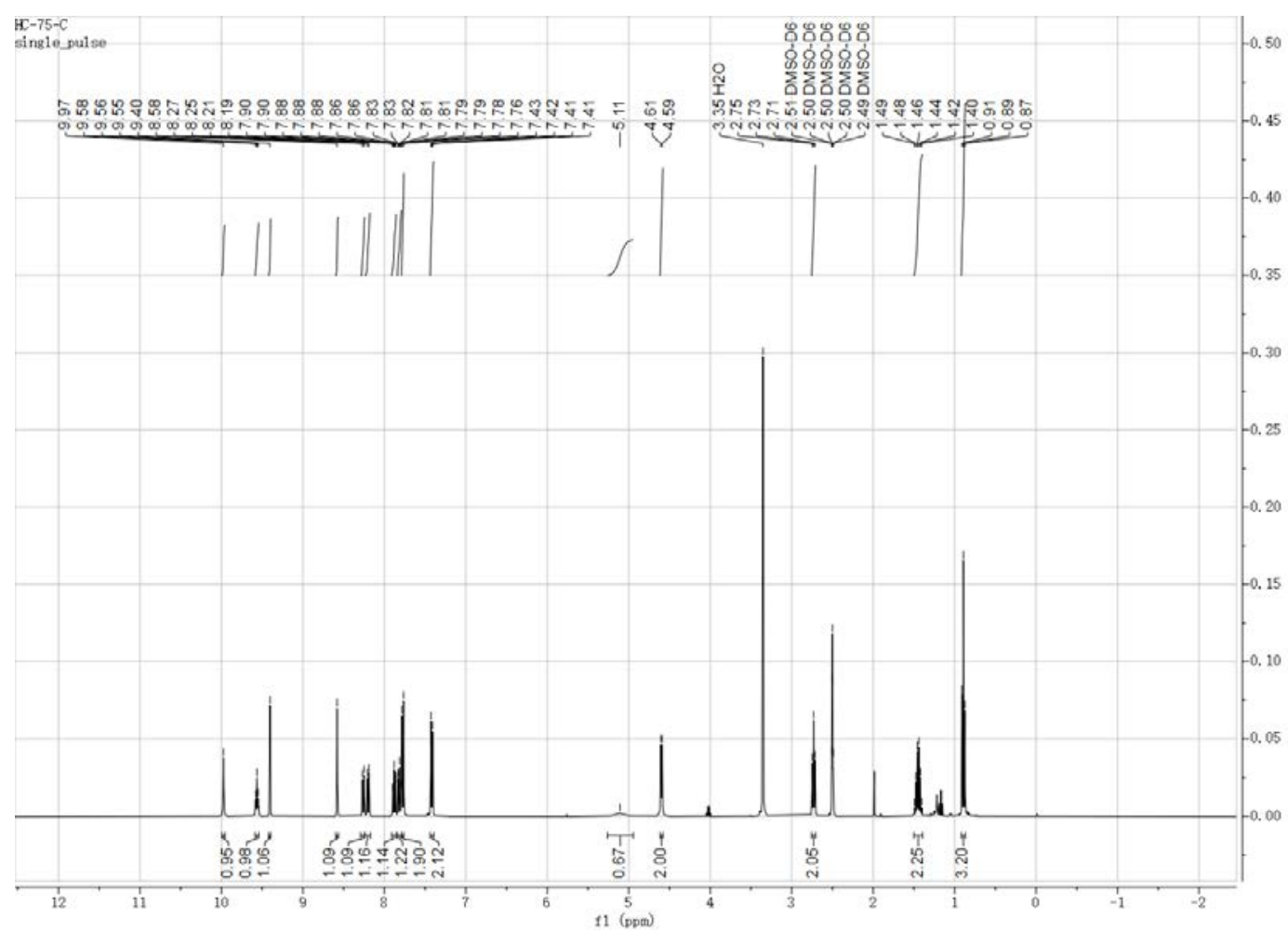

${ }^{1} \mathrm{H}-\mathrm{NMR}$ of 11c

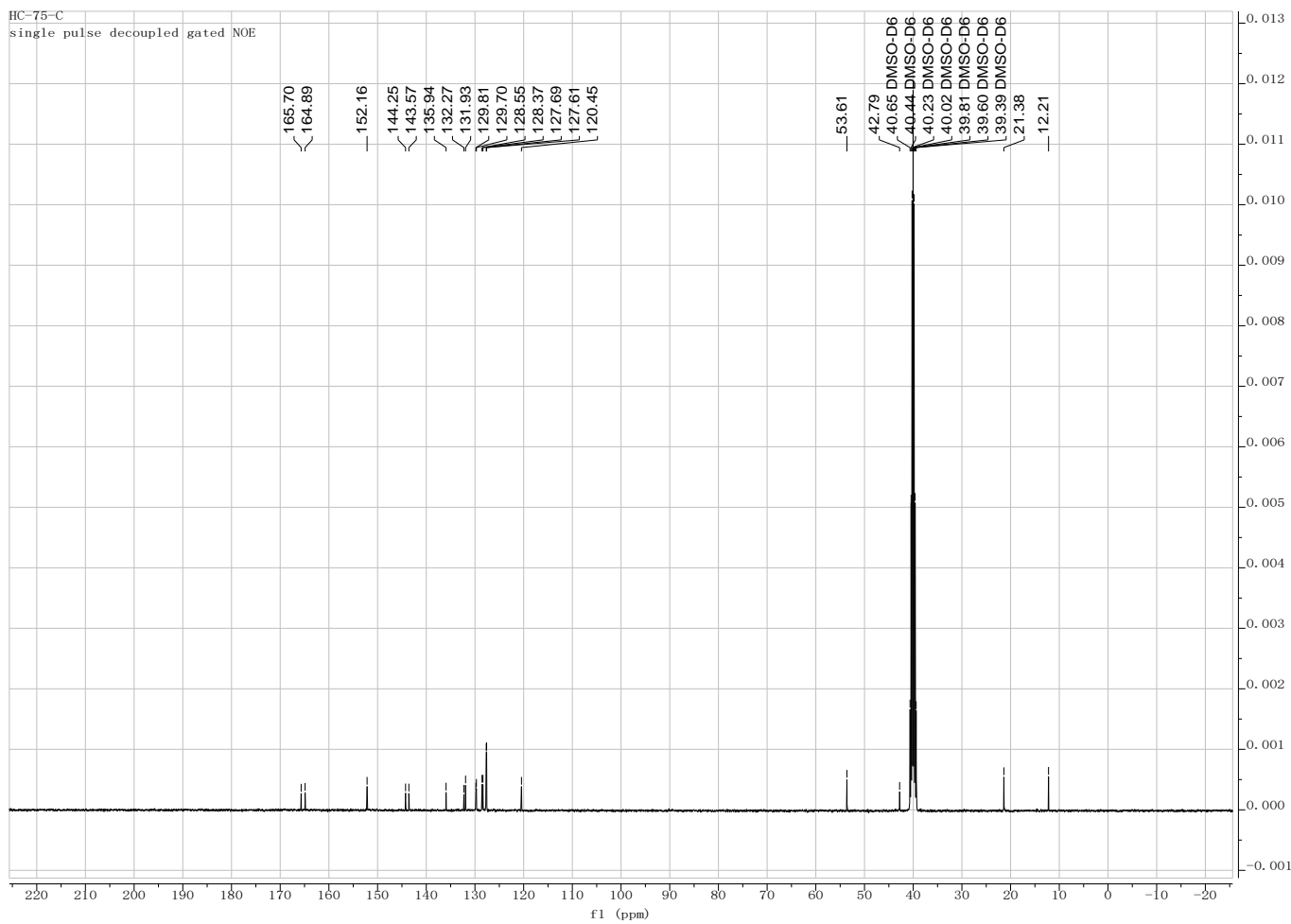

${ }^{13} \mathrm{C}-\mathrm{NMR}$ of $11 \mathrm{c}$ 

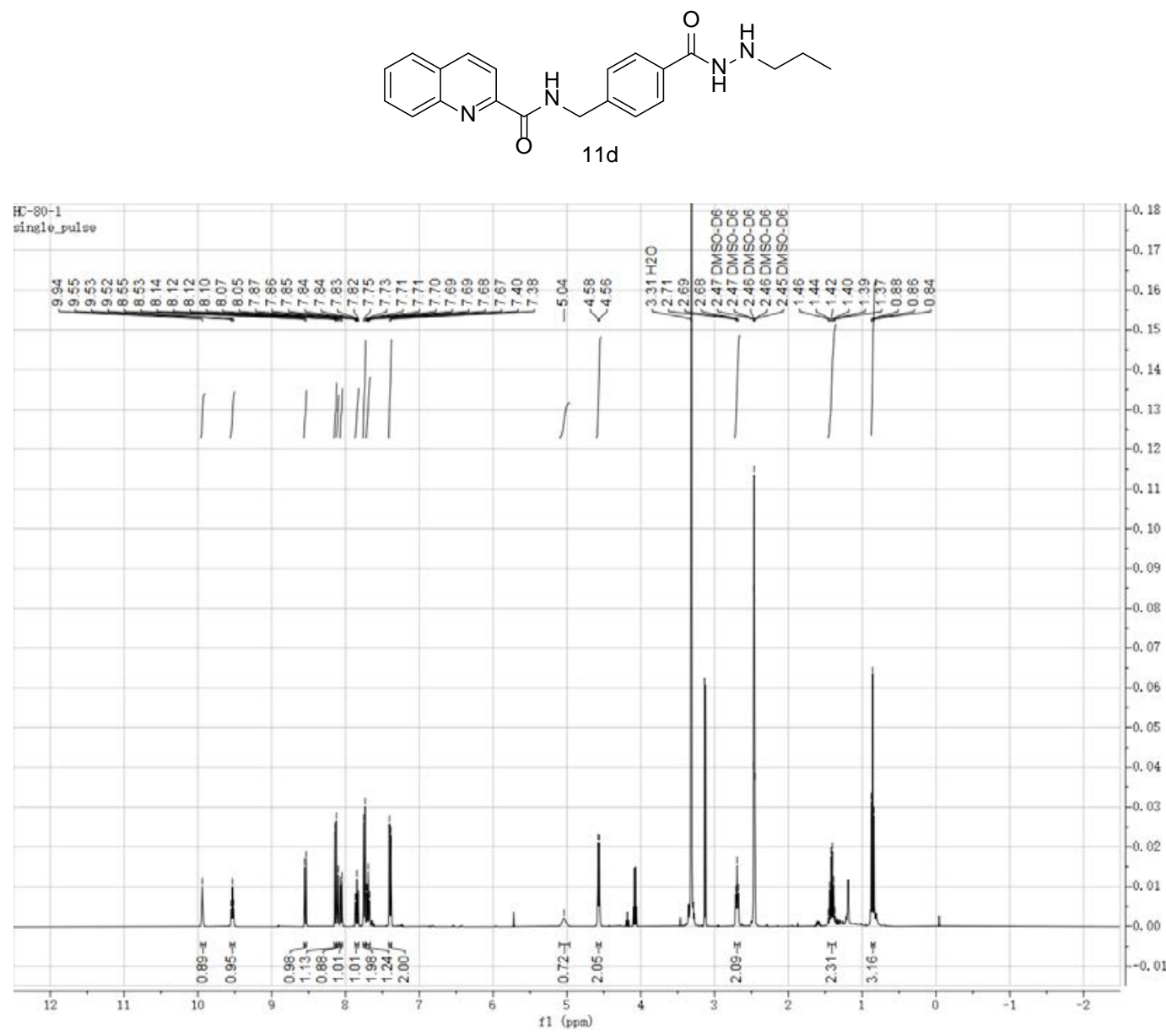

${ }^{1} \mathrm{H}-\mathrm{NMR}$ of 11d

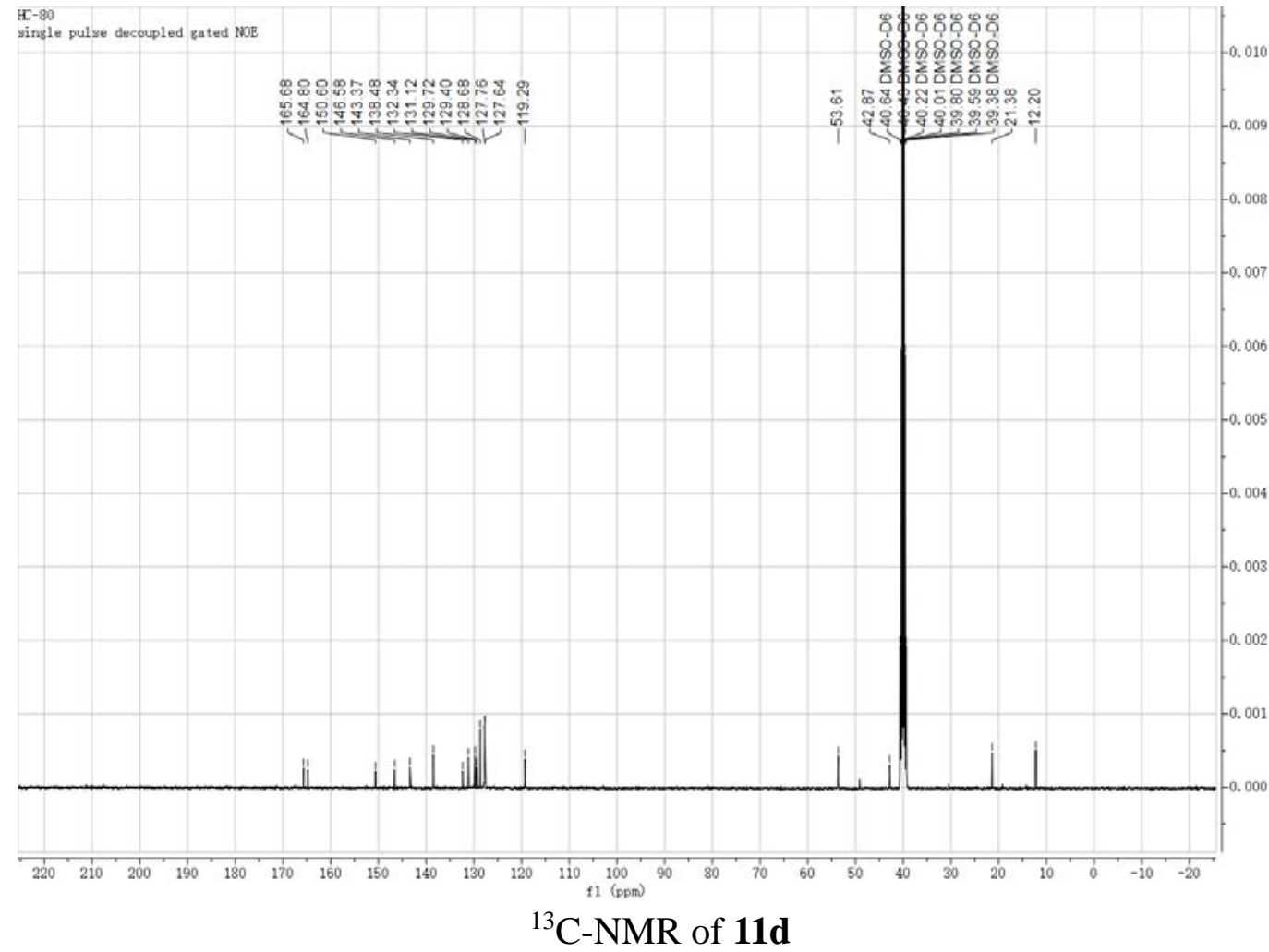



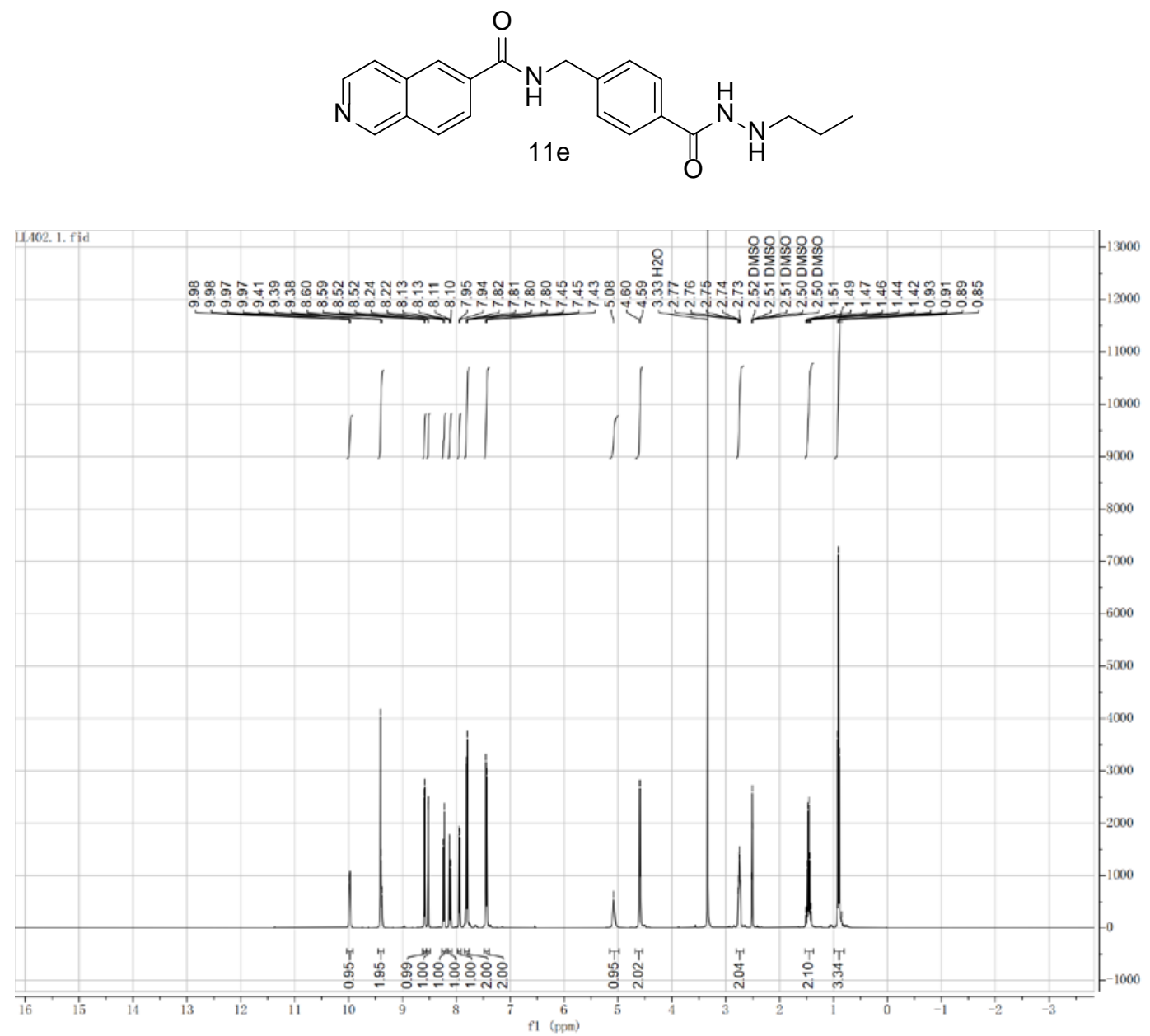

${ }^{1} \mathrm{H}-\mathrm{NMR}$ of 11e

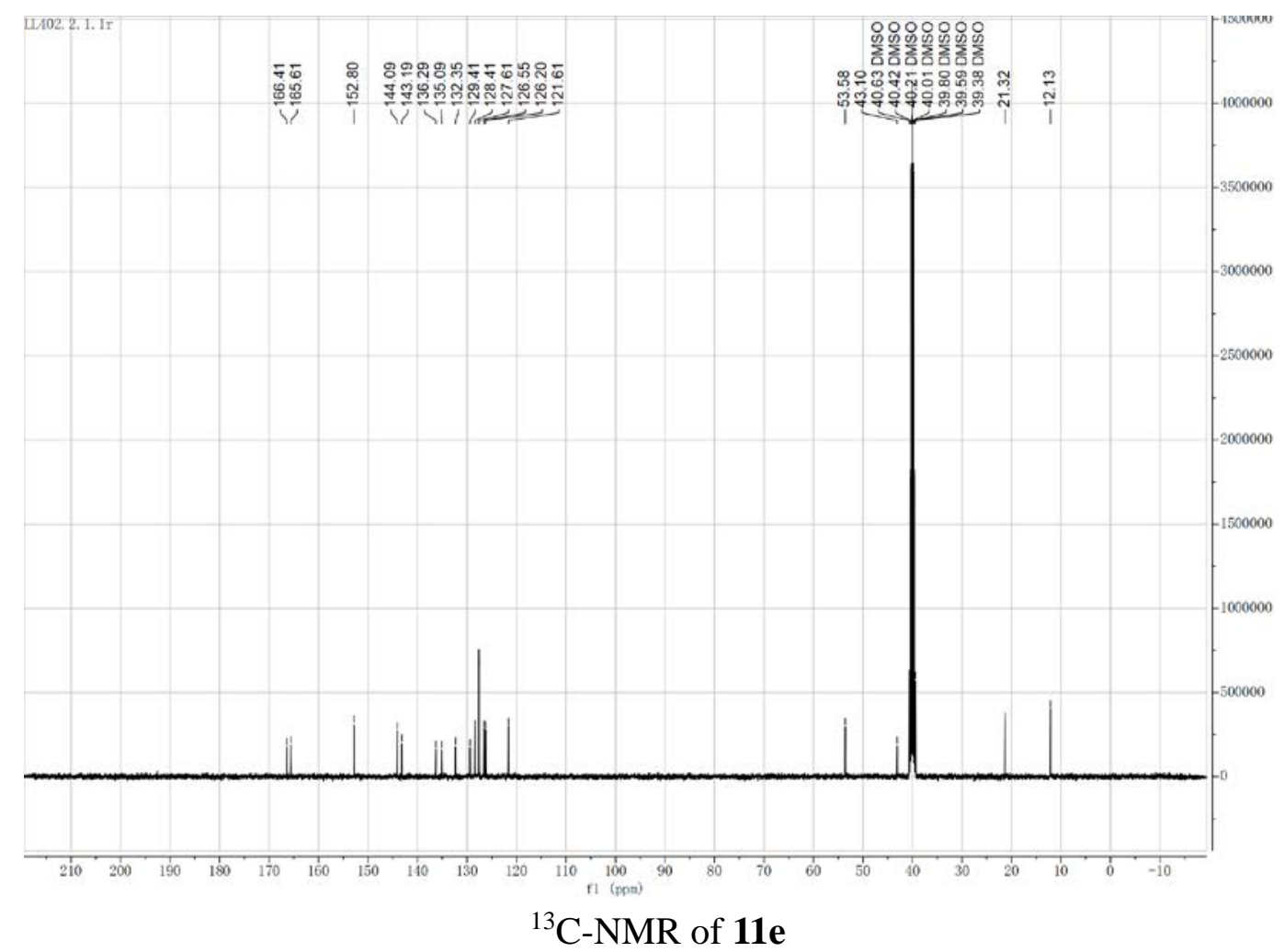



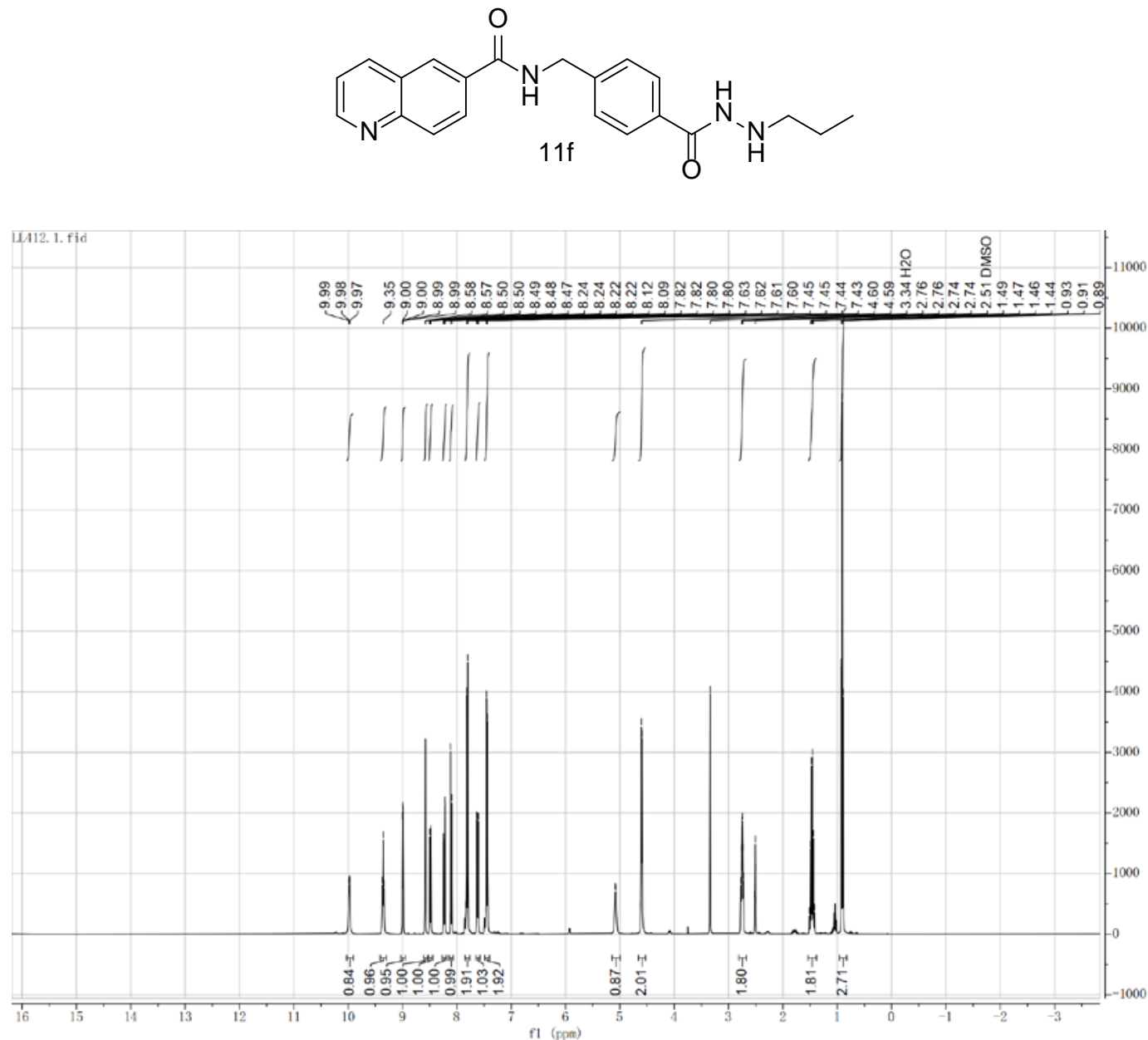

${ }^{1} \mathrm{H}-\mathrm{NMR}$ of $\mathbf{1 1 f}$

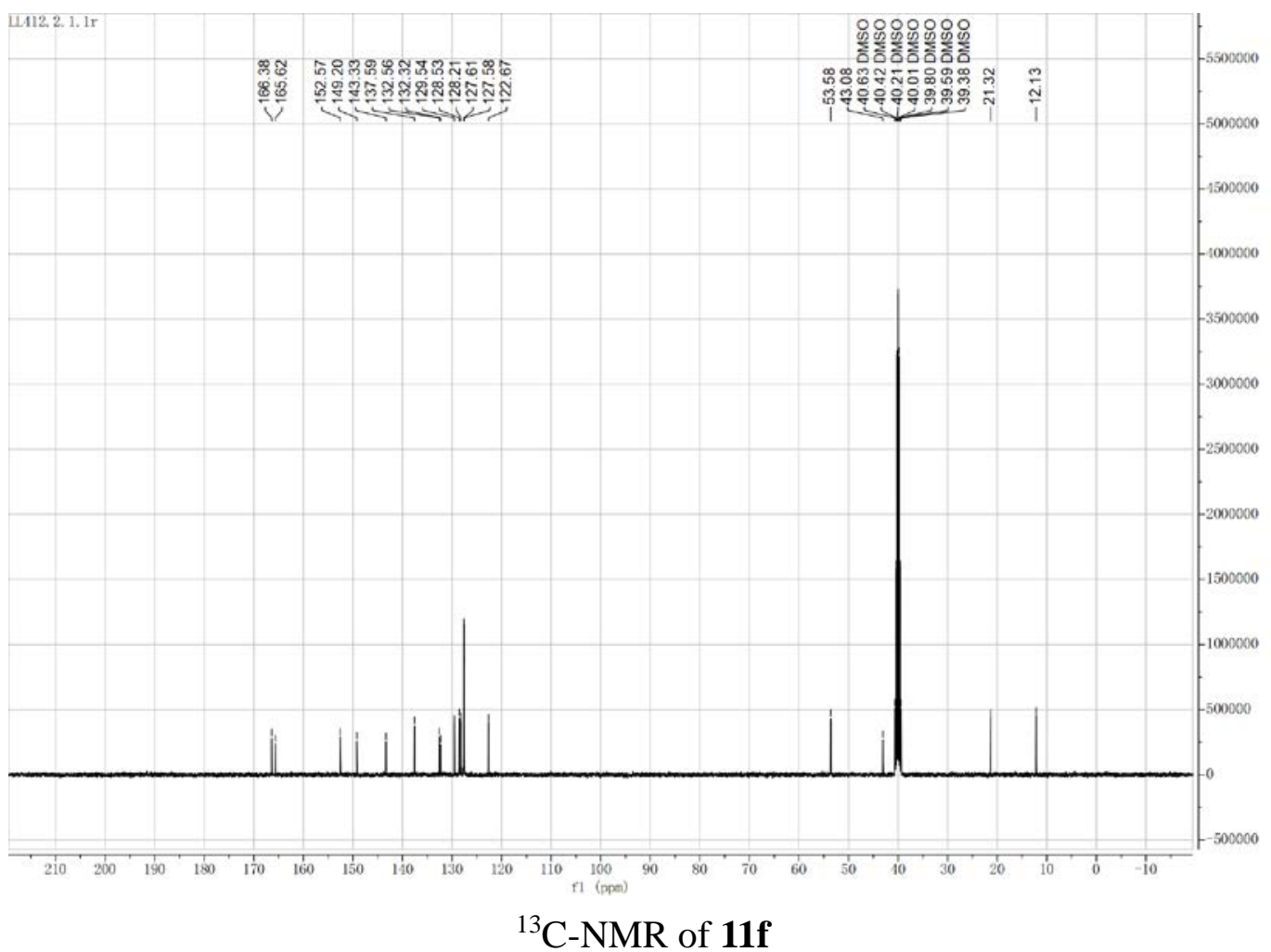



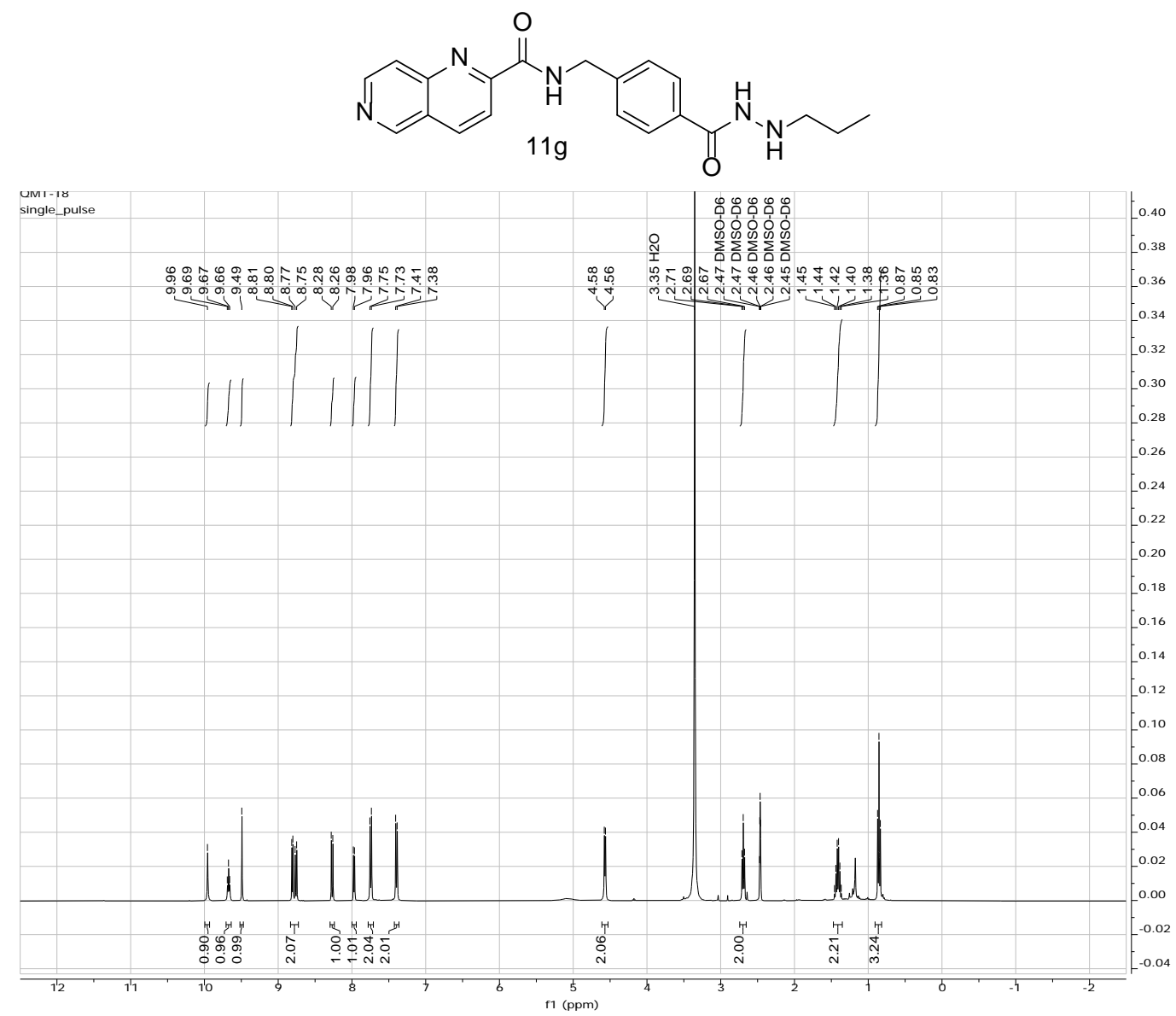

${ }^{1} \mathrm{H}-\mathrm{NMR}$ of $\mathbf{1 1 g}$

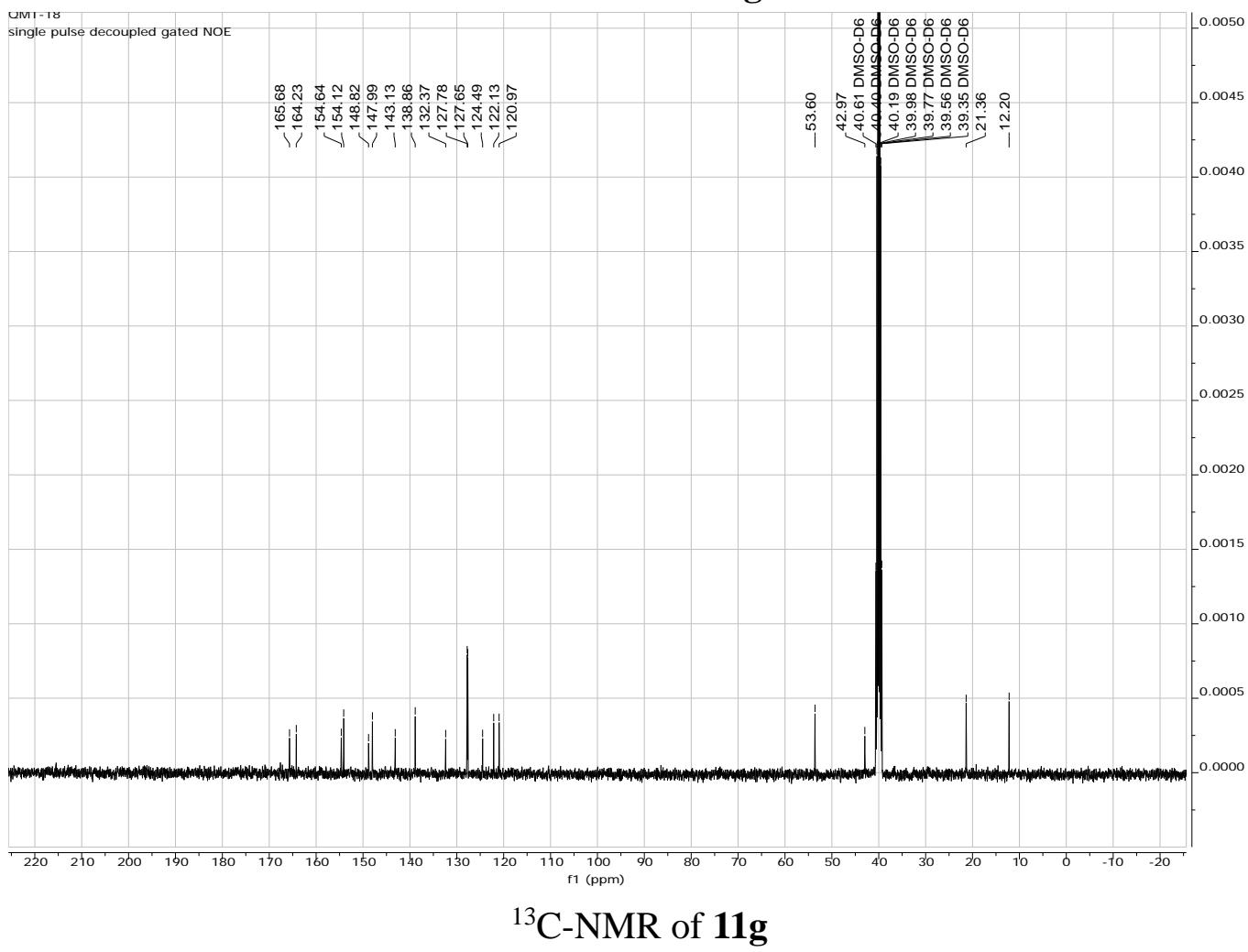



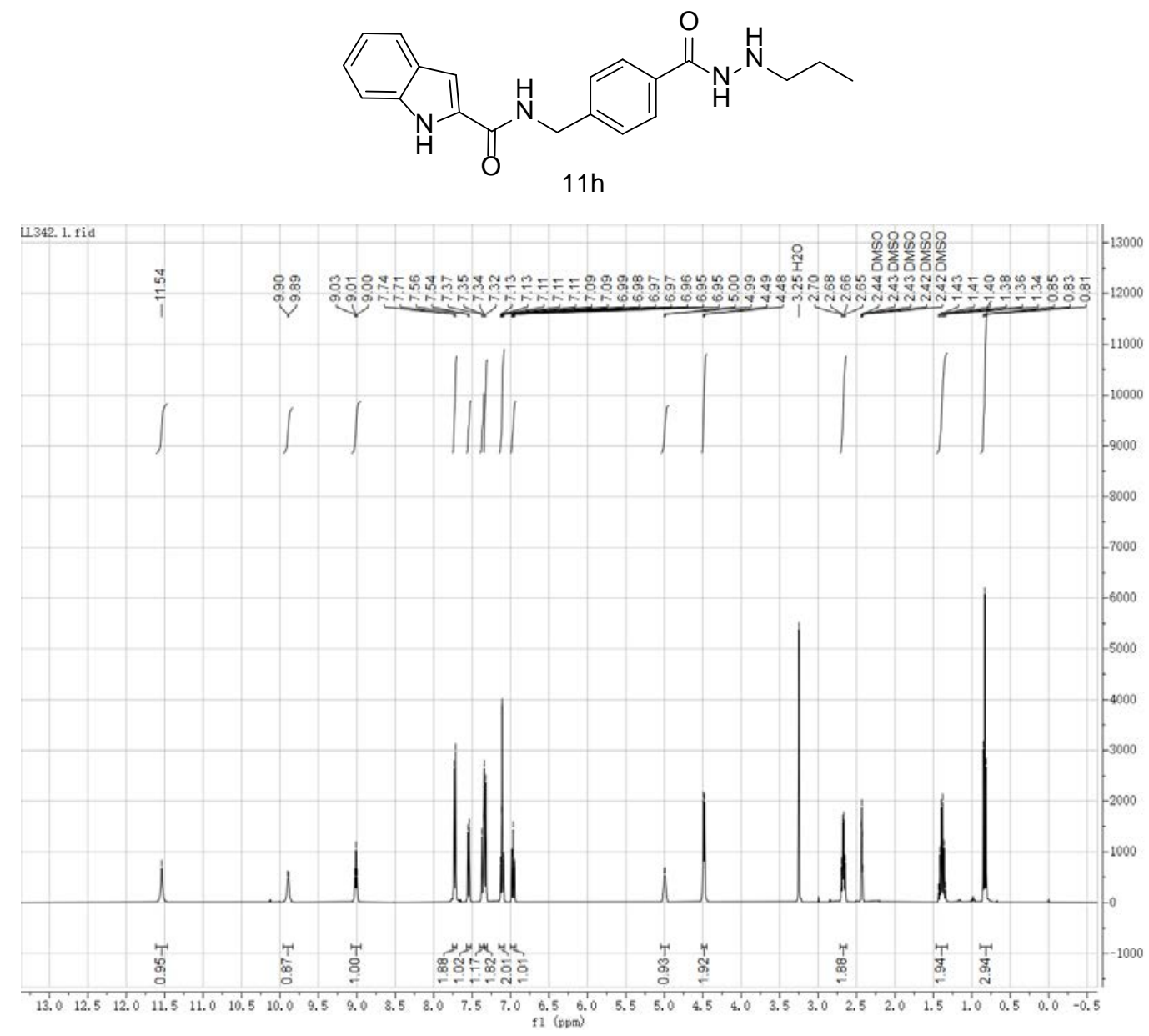

${ }^{1} \mathrm{H}-\mathrm{NMR}$ of $\mathbf{1 1 h}$

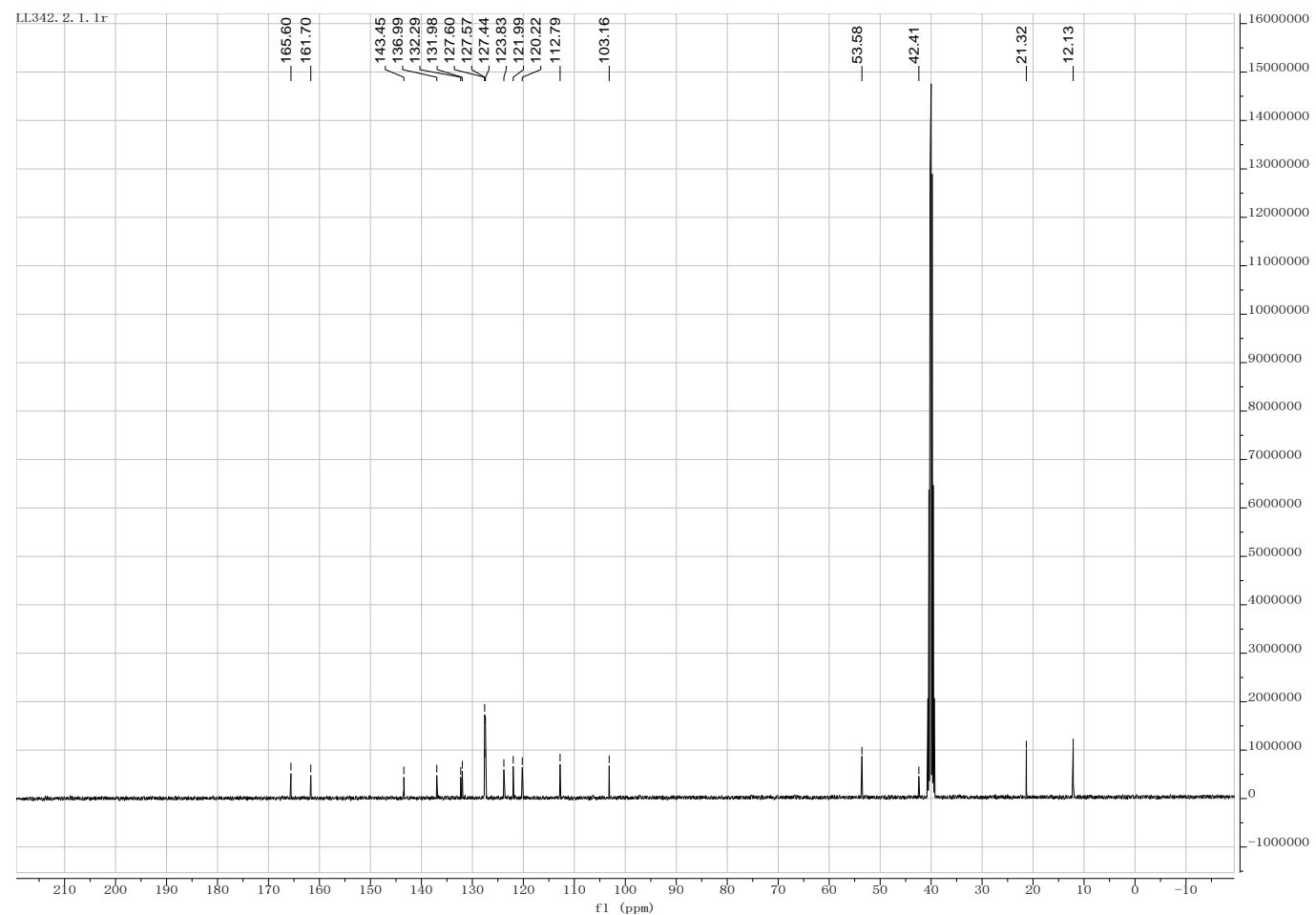

${ }^{13} \mathrm{C}-\mathrm{NMR}$ of $\mathbf{1 1 h}$ 

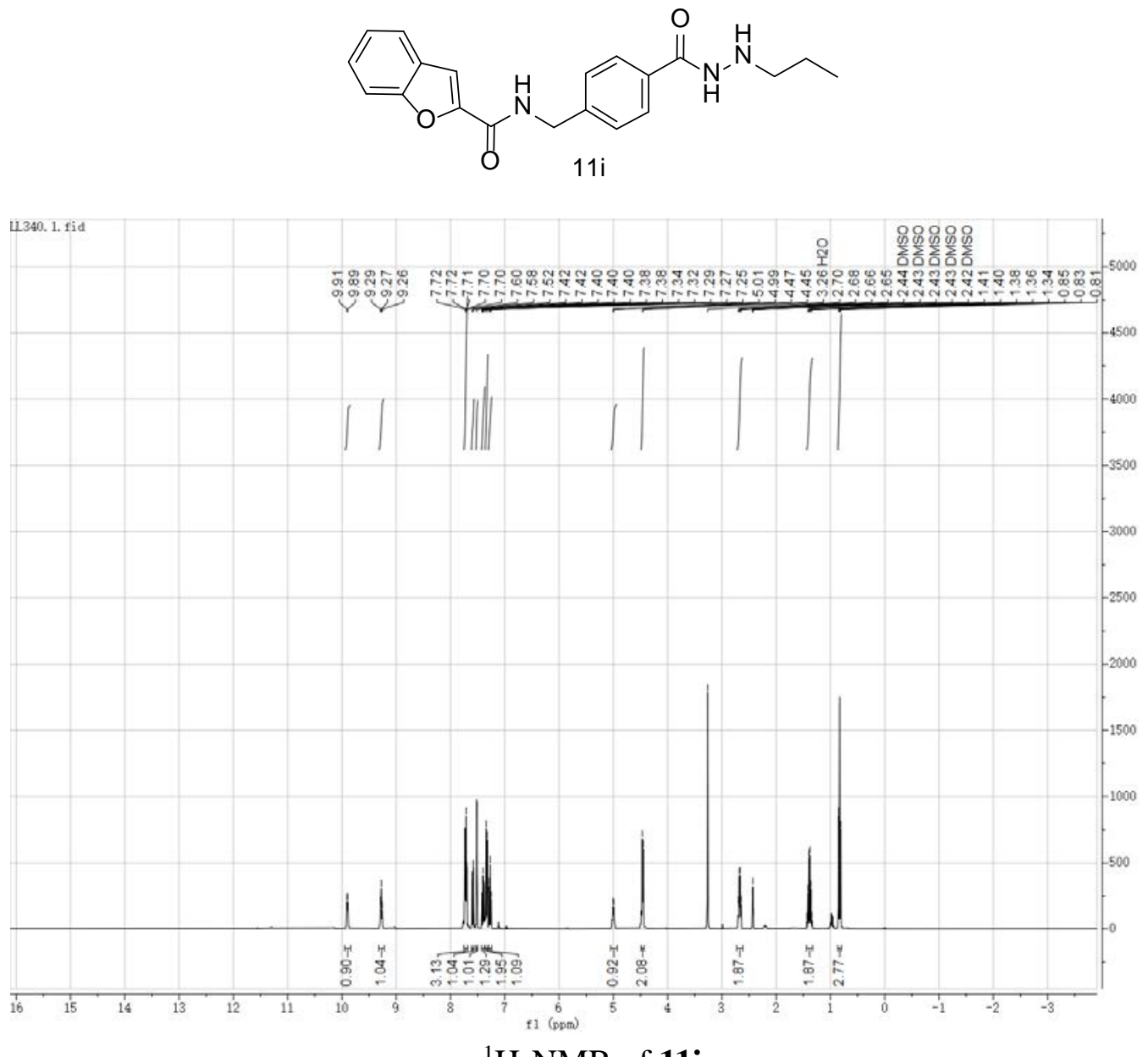

${ }^{1} \mathrm{H}-\mathrm{NMR}$ of 11i

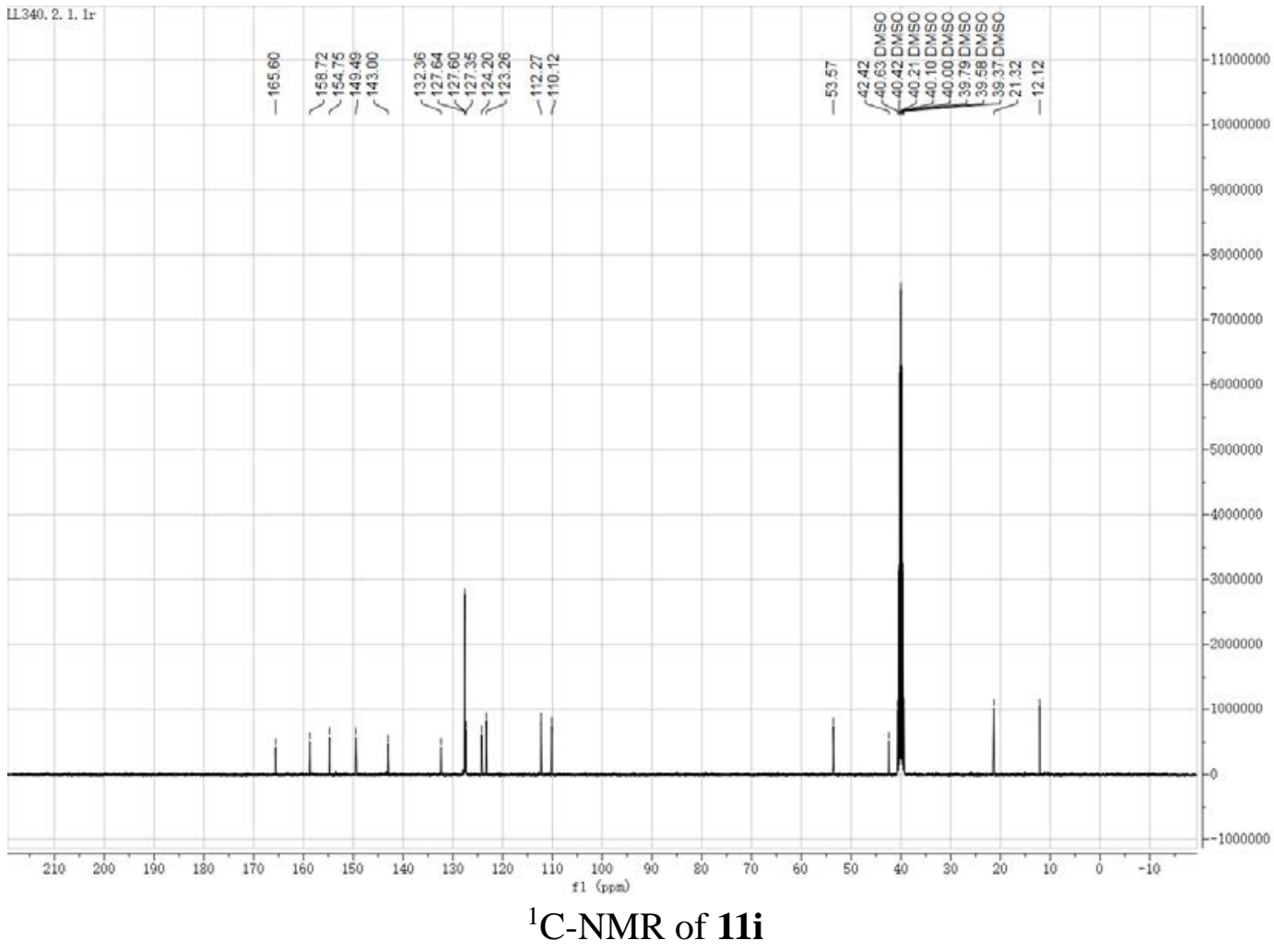


<smiles>CCCNNC(=O)c1ccc(CNC(=O)c2cc3ccccc3s2)cc1</smiles>

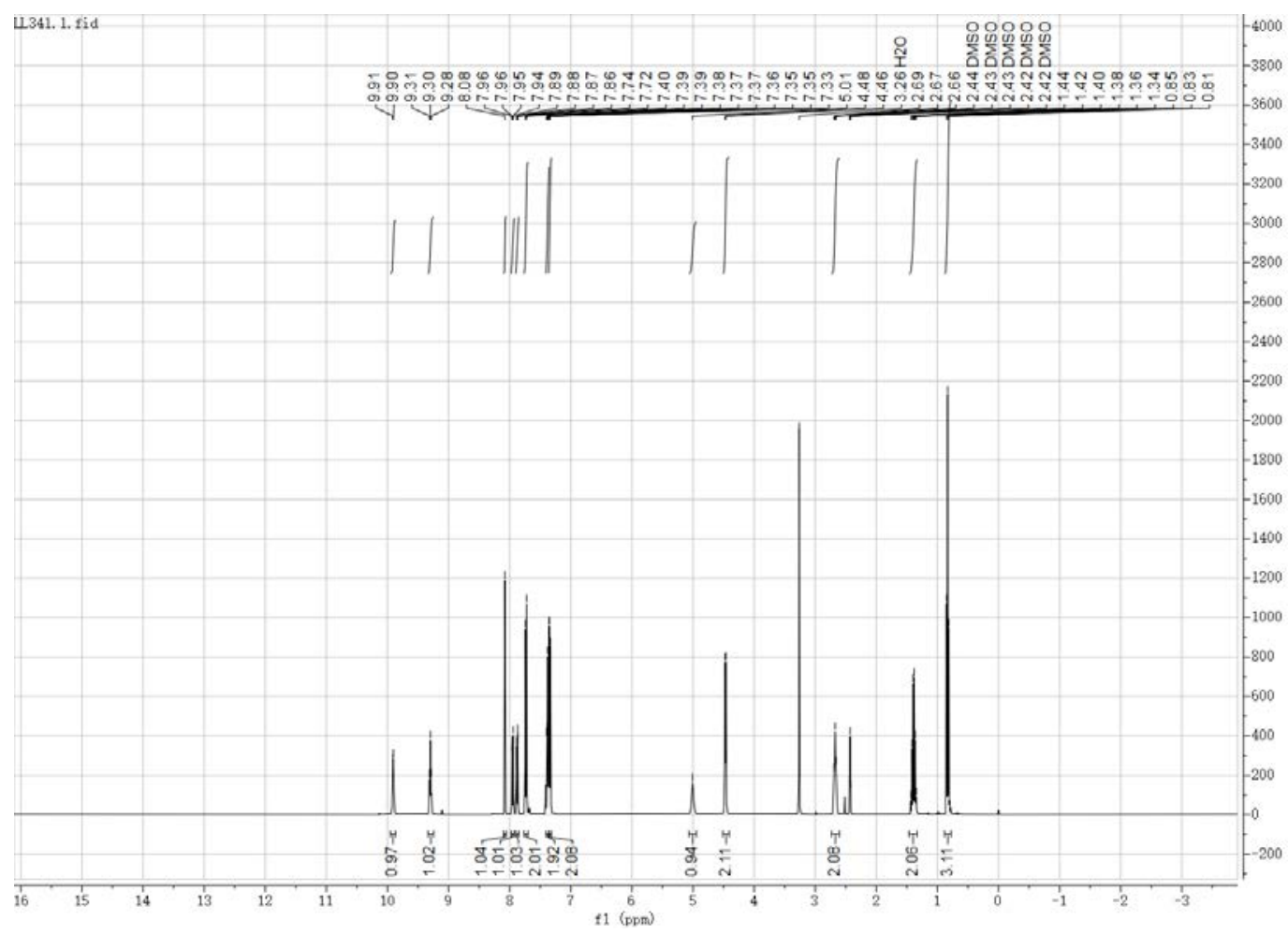

${ }^{1} \mathrm{H}-\mathrm{NMR}$ of $\mathbf{1 1} \mathbf{j}$

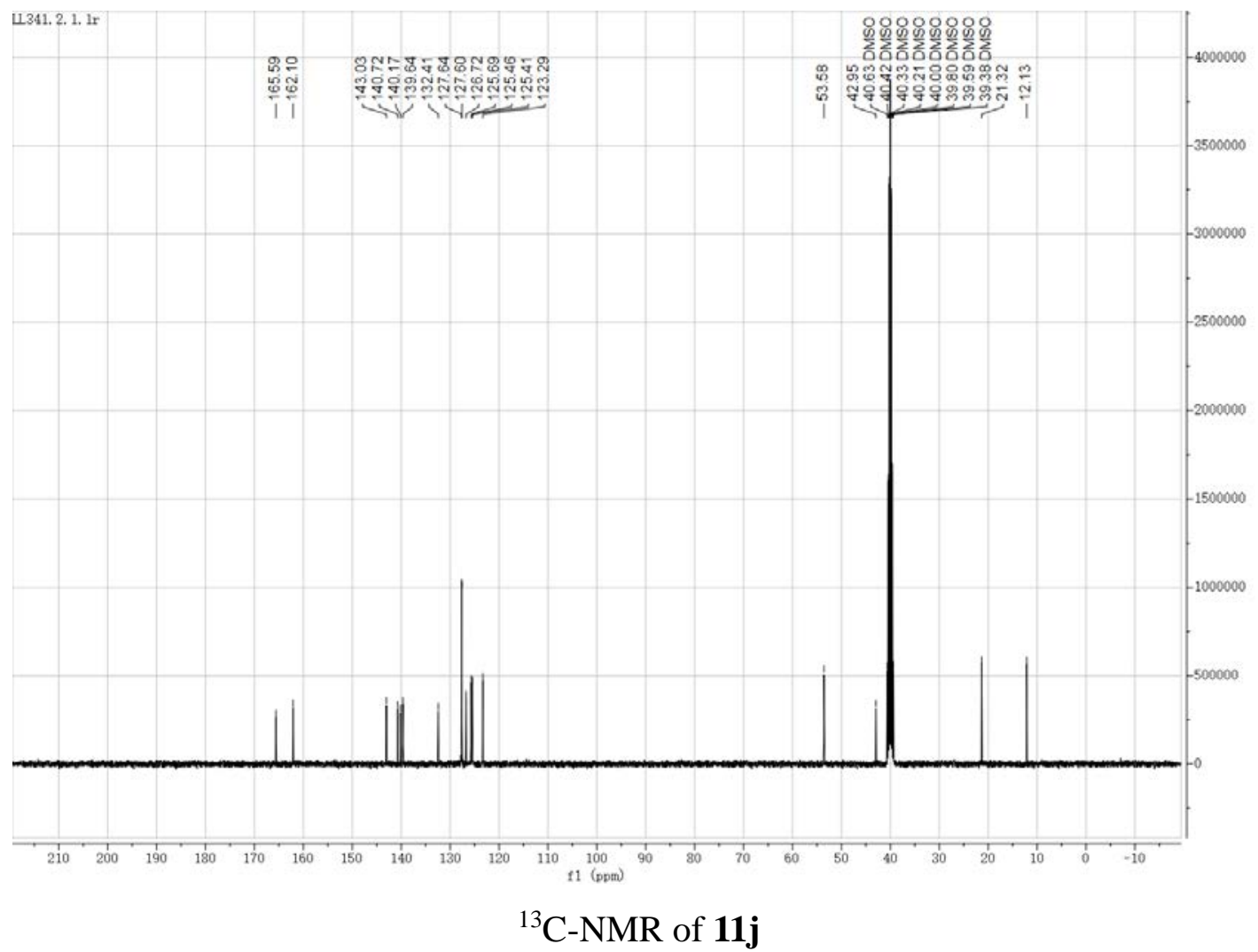



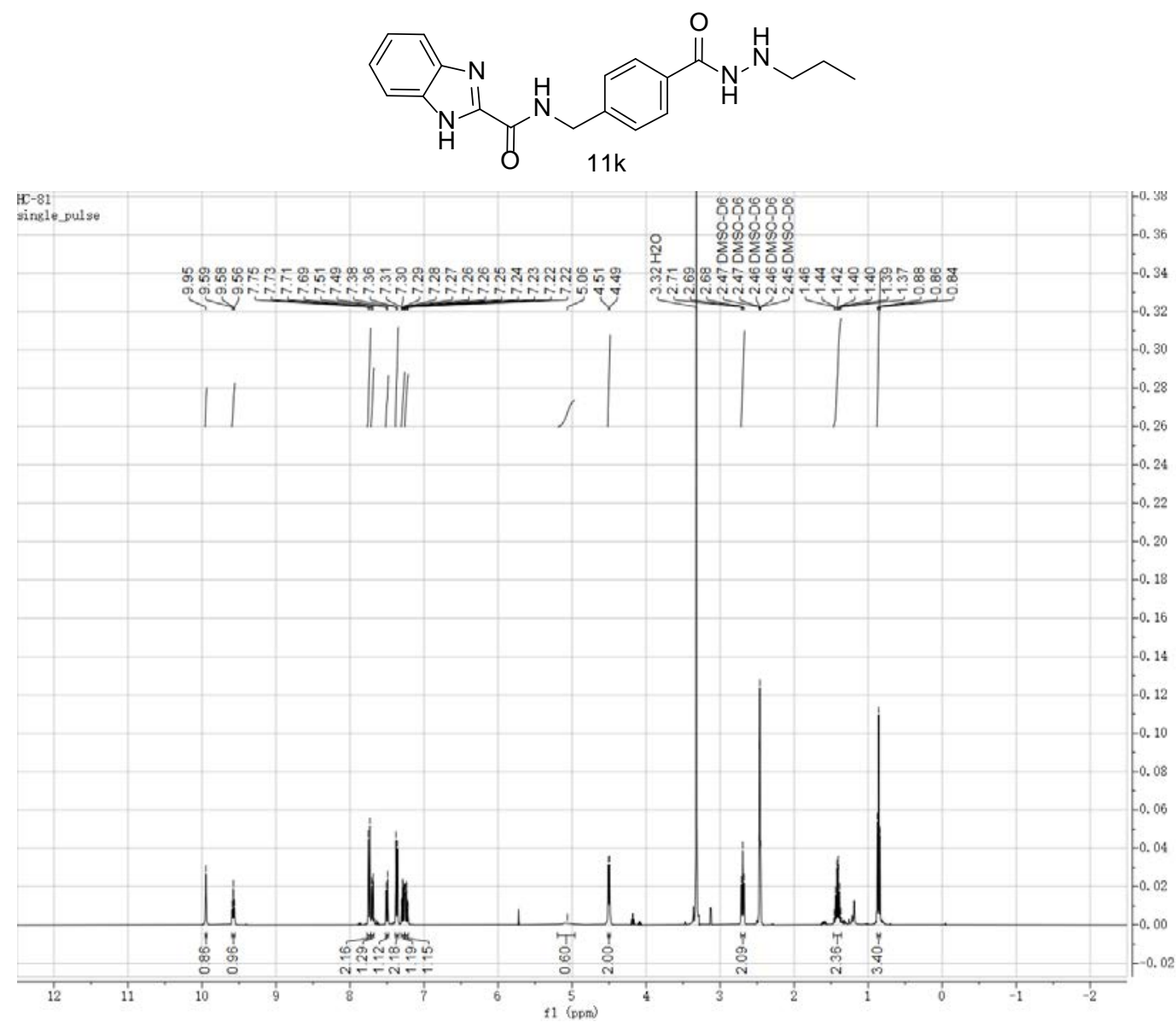

${ }^{1} \mathrm{H}-\mathrm{NMR}$ of $\mathbf{1 1 k}$

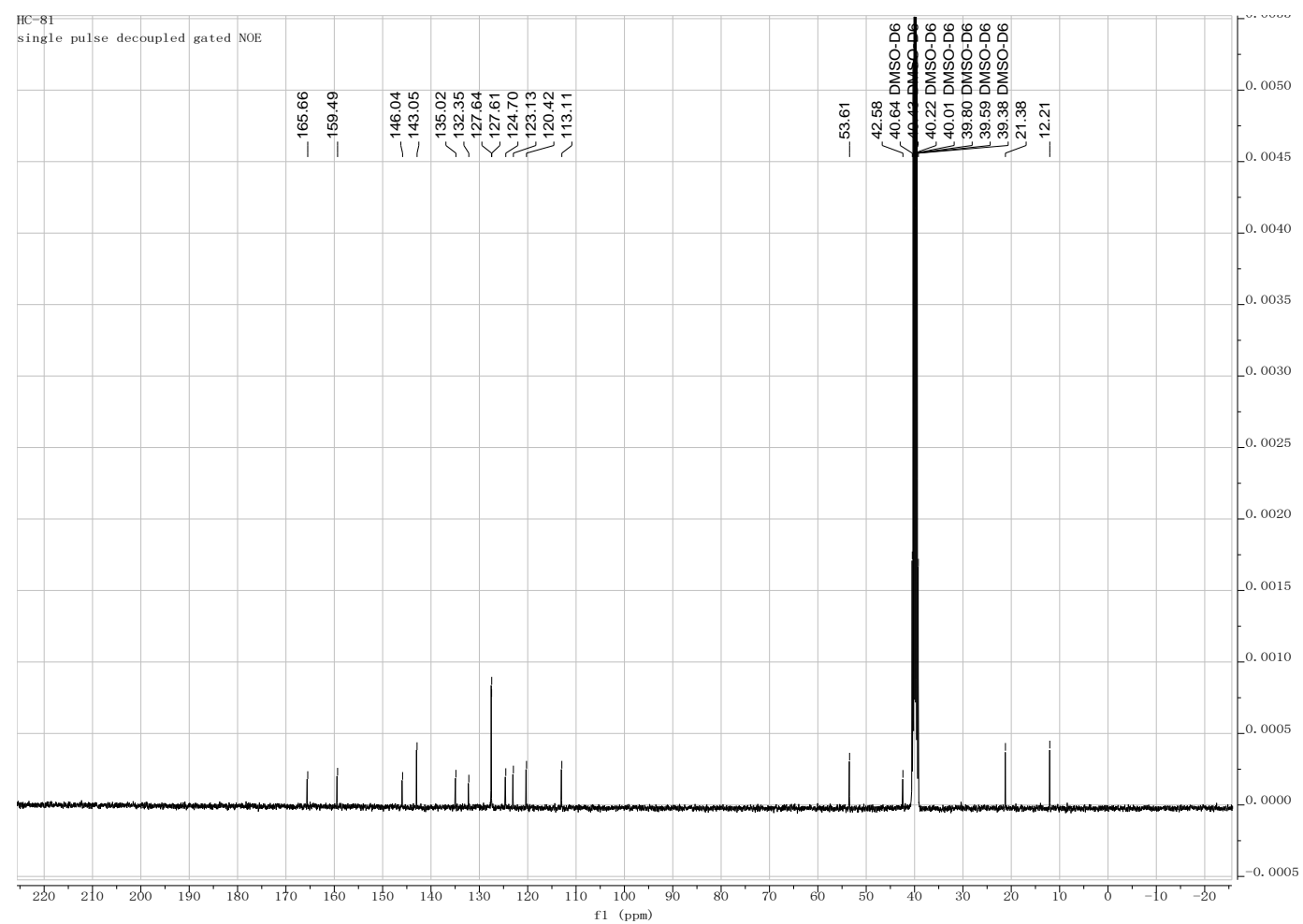

${ }^{13} \mathrm{C}-\mathrm{NMR}$ of $\mathbf{1 1 k}$ 

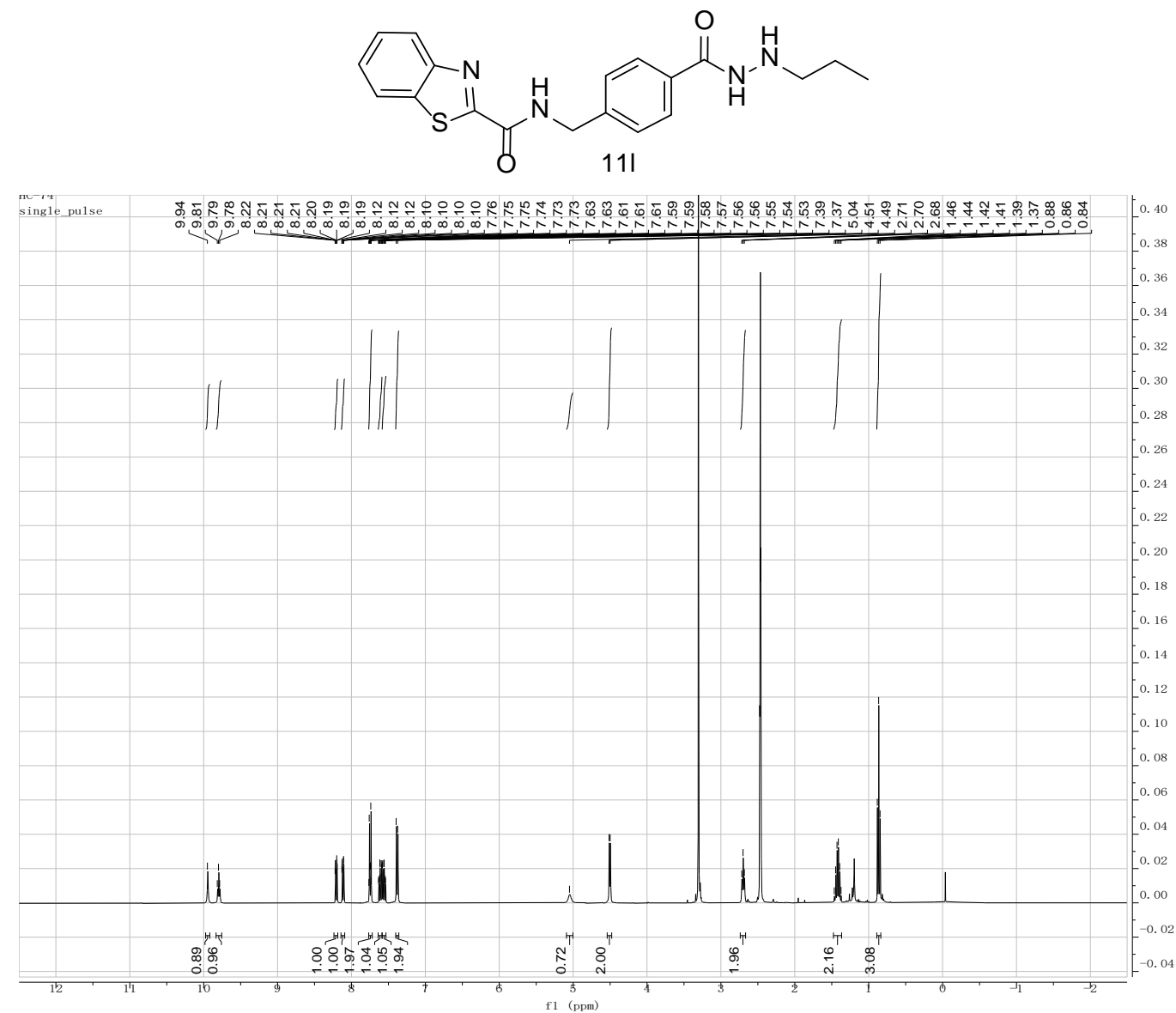

${ }^{1} \mathrm{H}-\mathrm{NMR}$ of $\mathbf{1 1 l}$

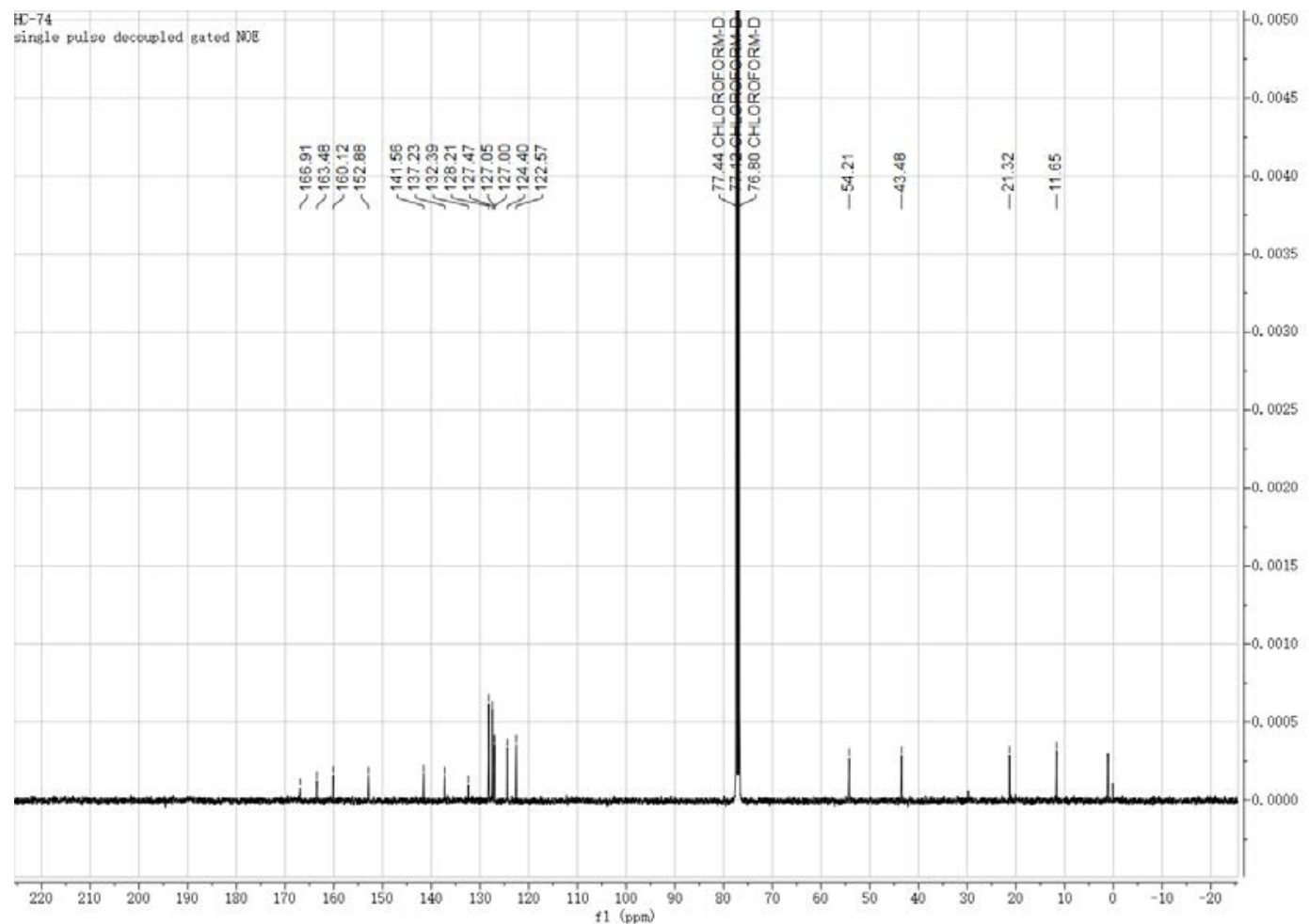

${ }^{13} \mathrm{C}-\mathrm{NMR}$ of $11 \mathrm{I}$ 

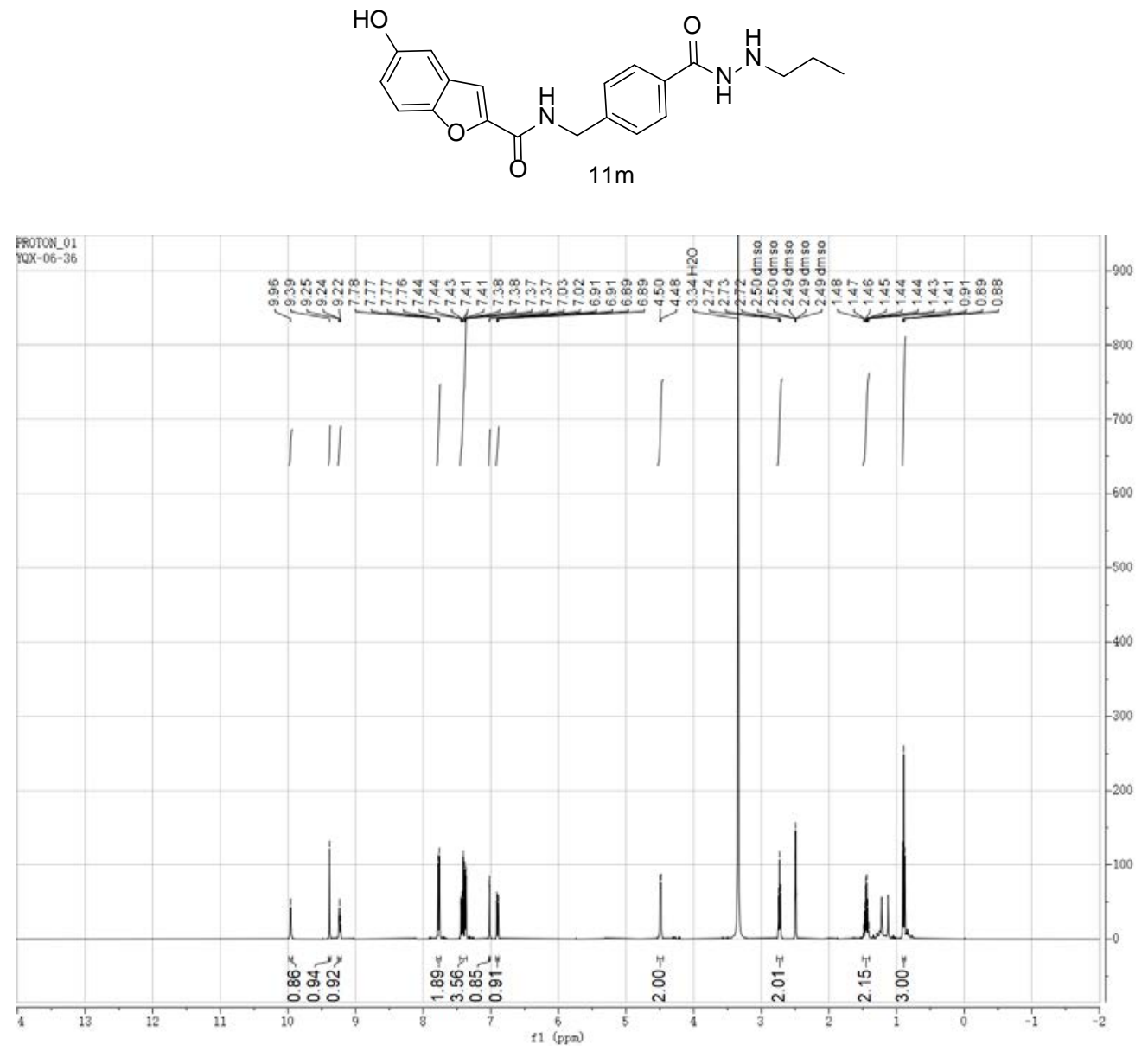

${ }^{1} \mathrm{H}-\mathrm{NMR}$ of $11 \mathrm{~m}$

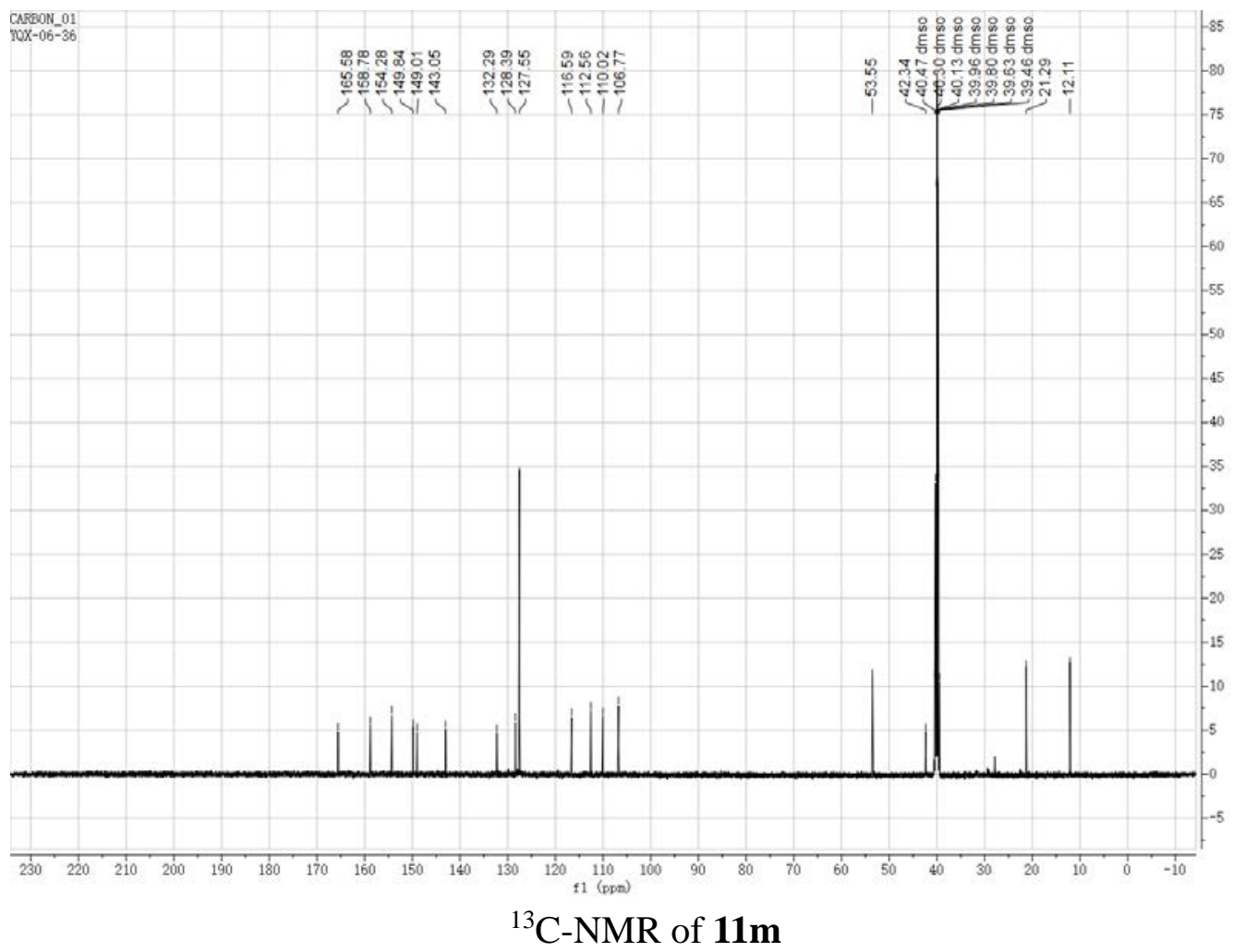



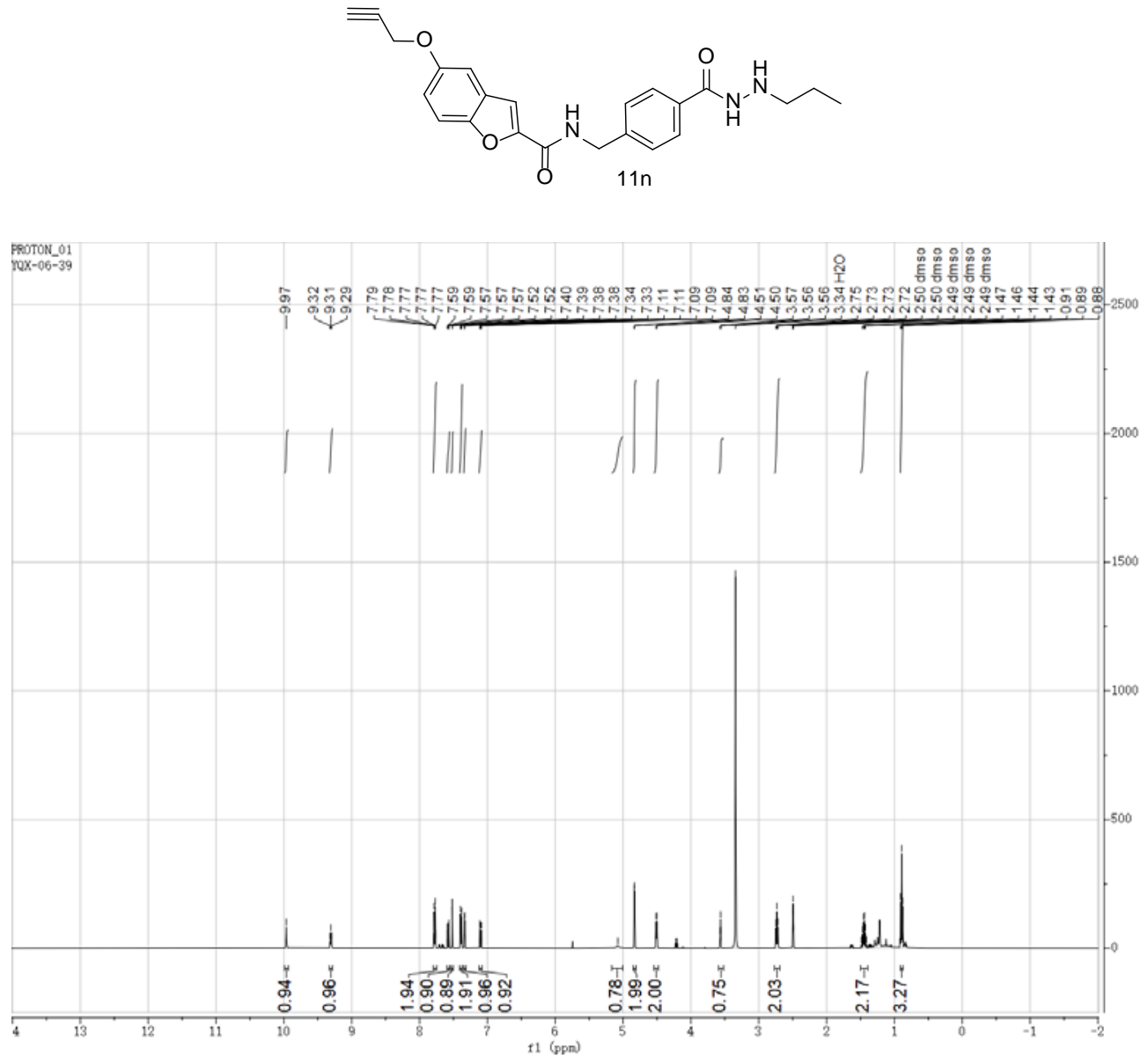

${ }^{1} \mathrm{H}-\mathrm{NMR}$ of $11 \mathrm{n}$

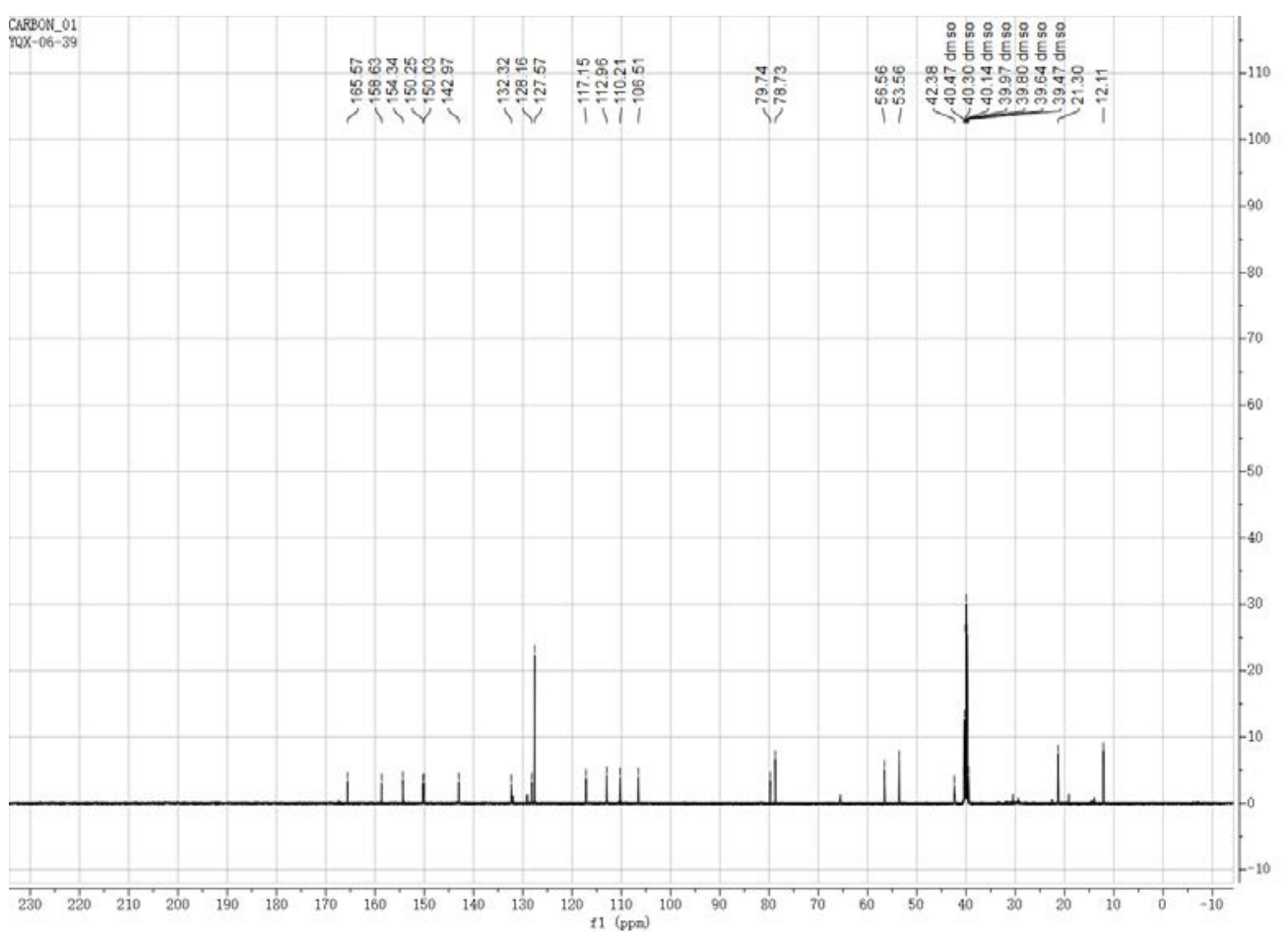

${ }^{13} \mathrm{C}-\mathrm{NMR}$ of 11n 

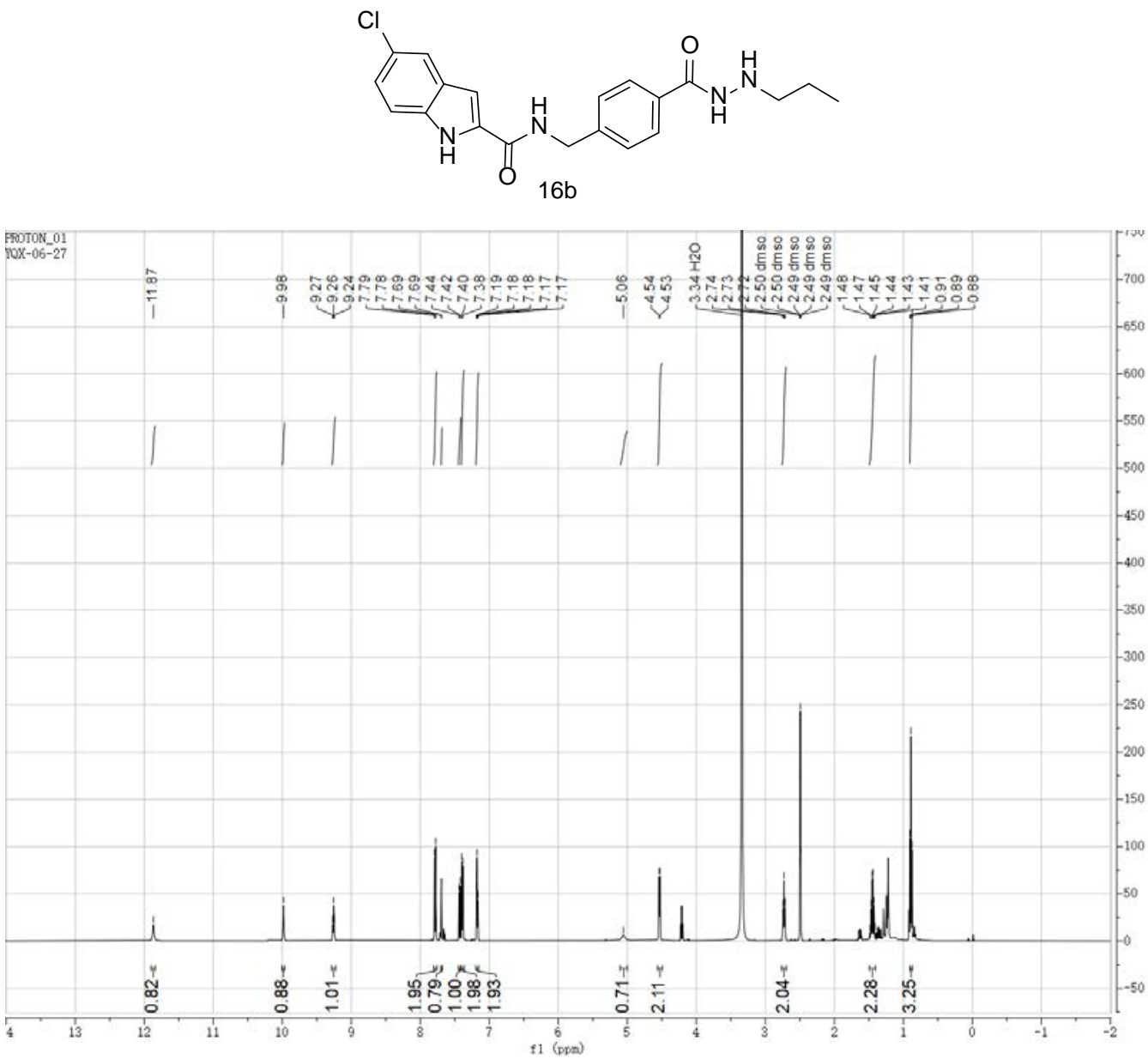

${ }^{1} \mathrm{H}-\mathrm{NMR}$ of $\mathbf{1 6 b}$

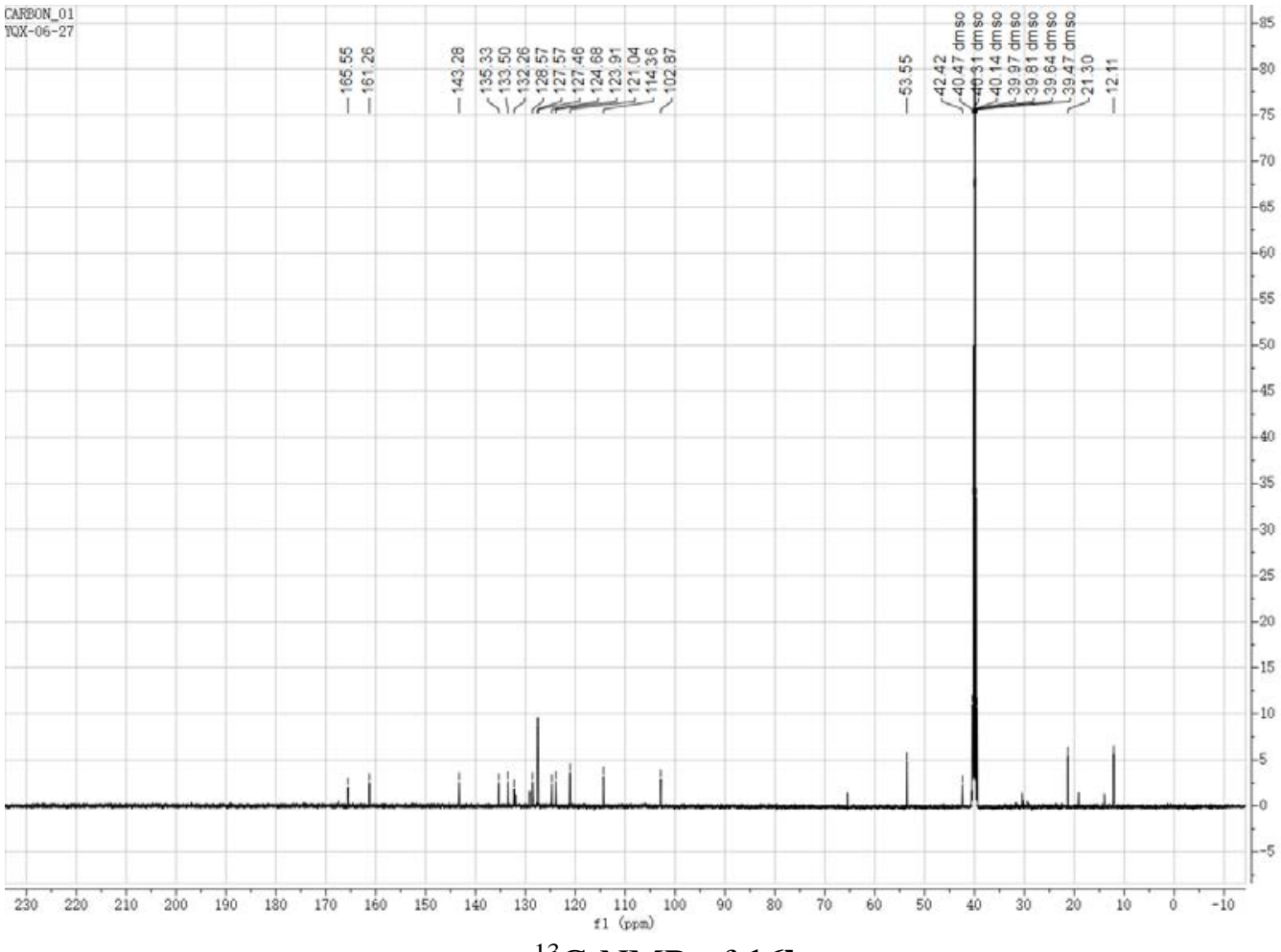

${ }^{13} \mathrm{C}-\mathrm{NMR}$ of $\mathbf{1 6 b}$ 

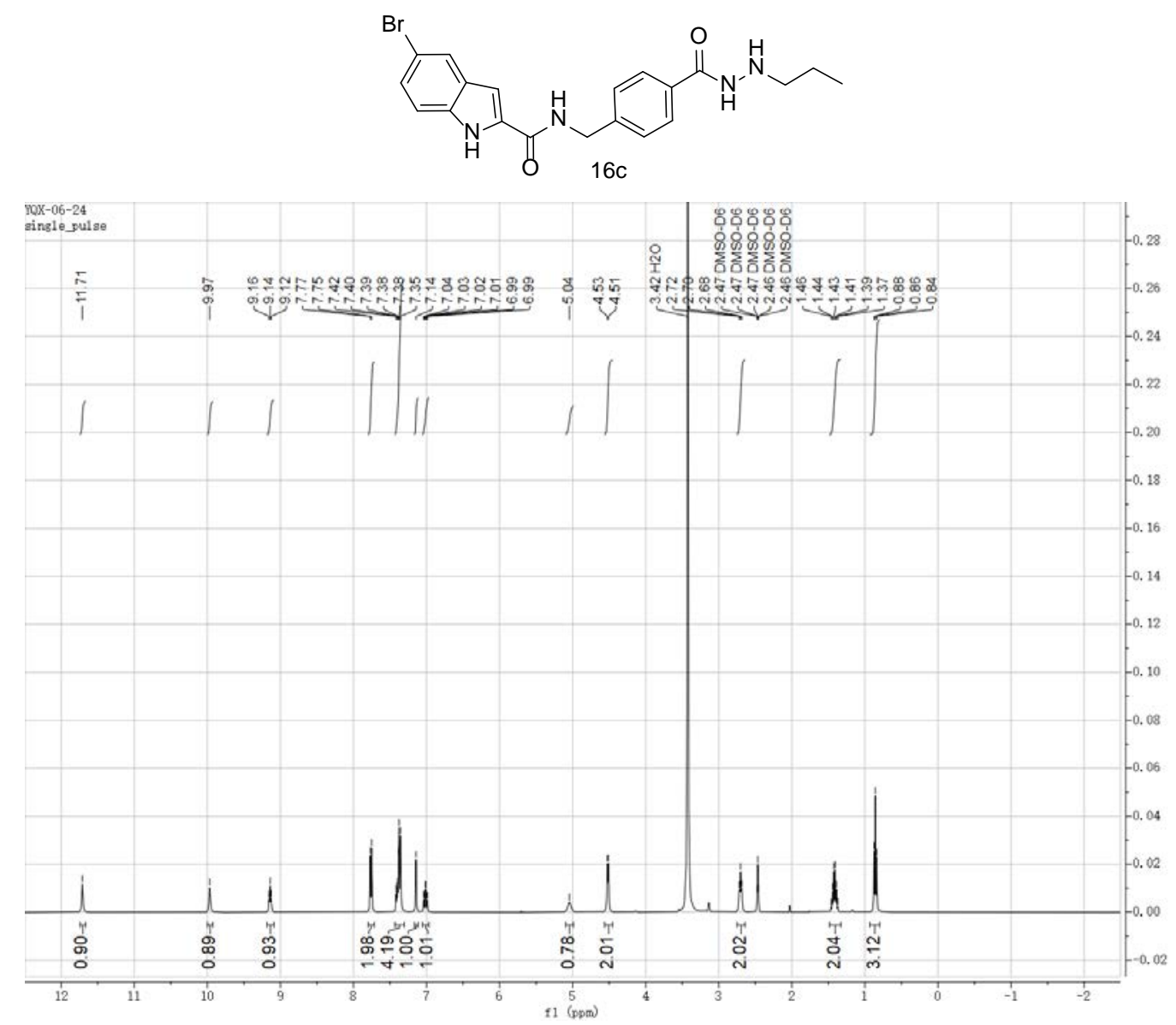

${ }^{1} \mathrm{H}-\mathrm{NMR}$ of $\mathbf{1 6 c}$

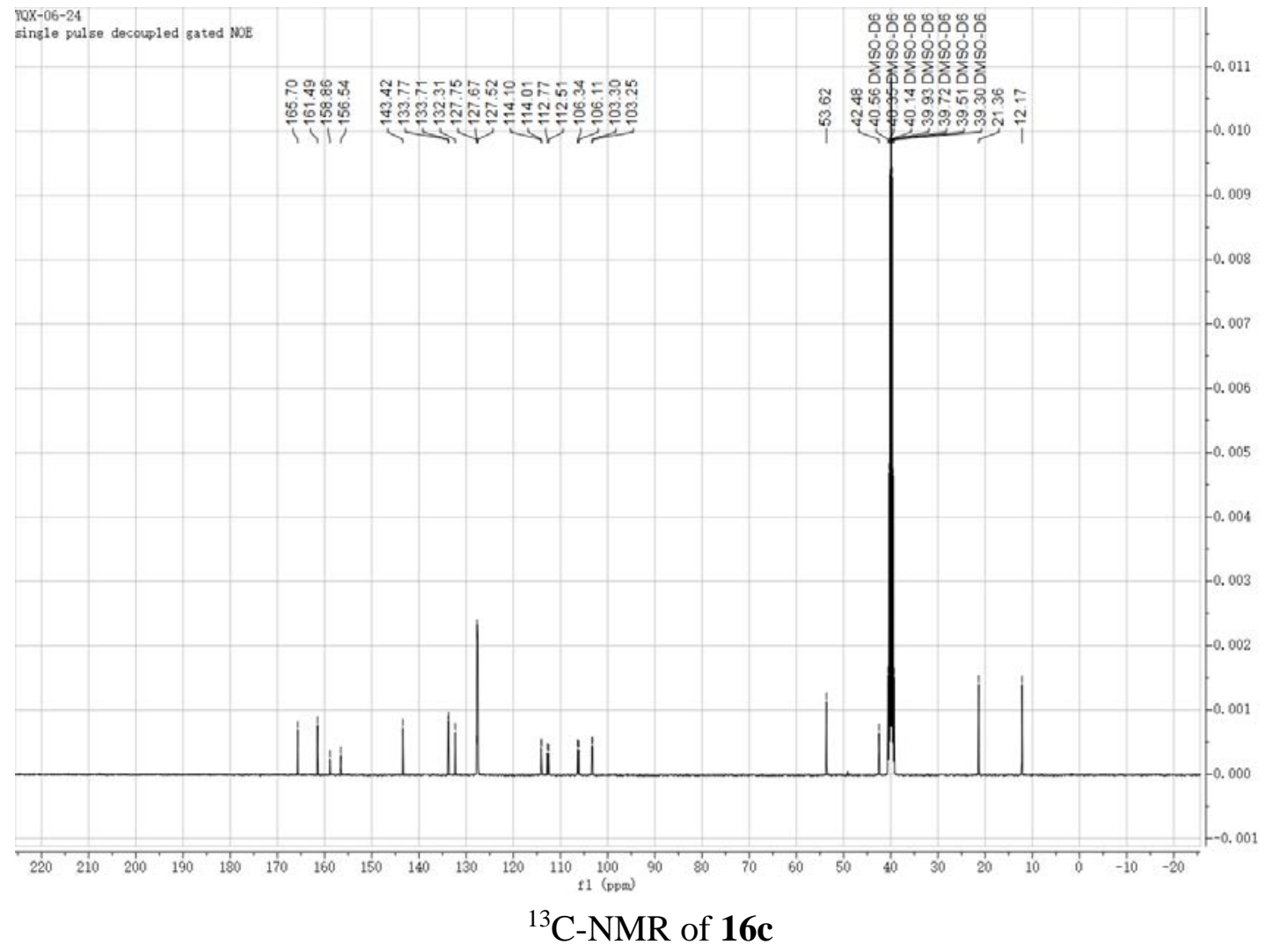



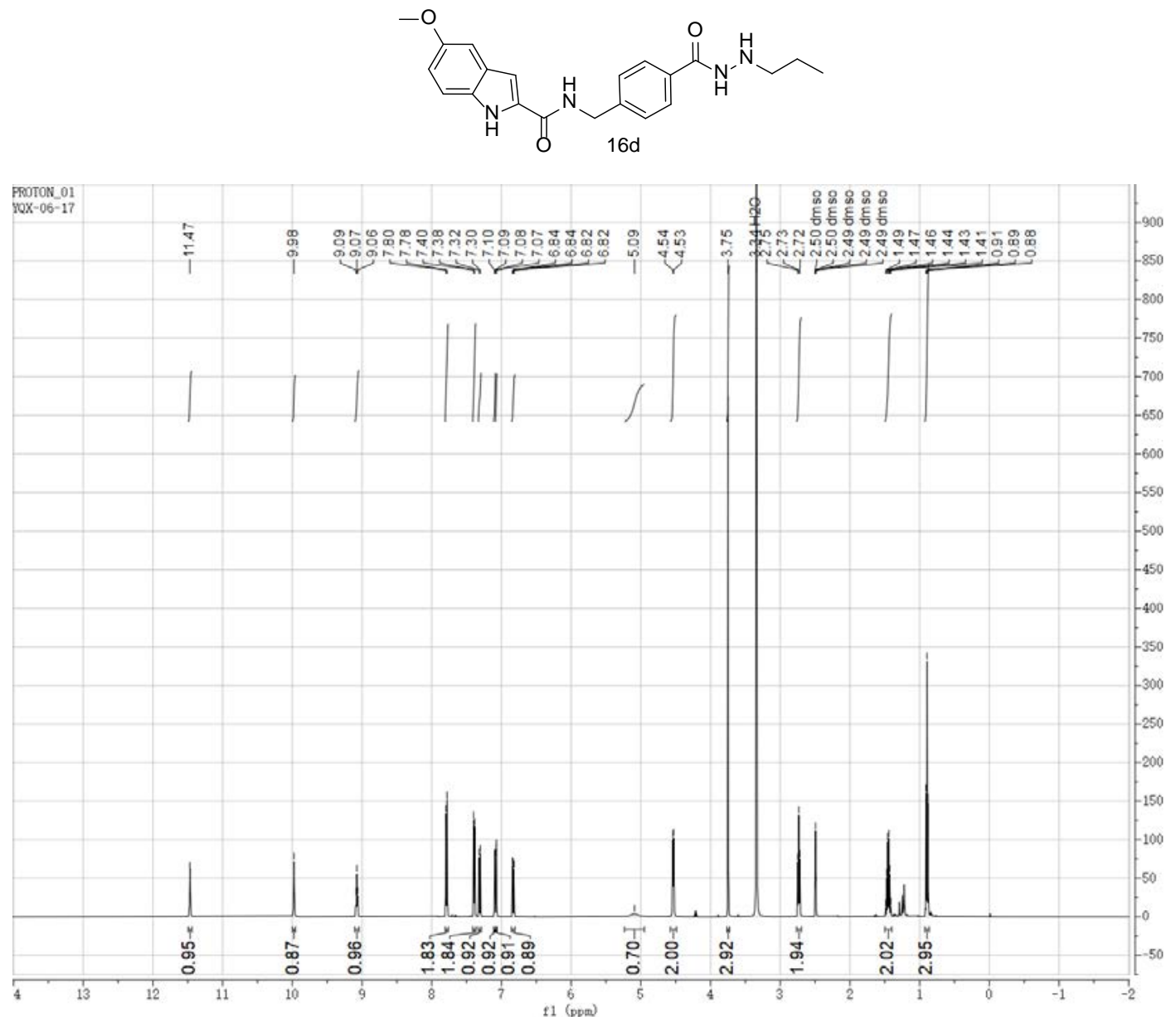

${ }^{1} \mathrm{H}-\mathrm{NMR}$ of $\mathbf{1 6 d}$

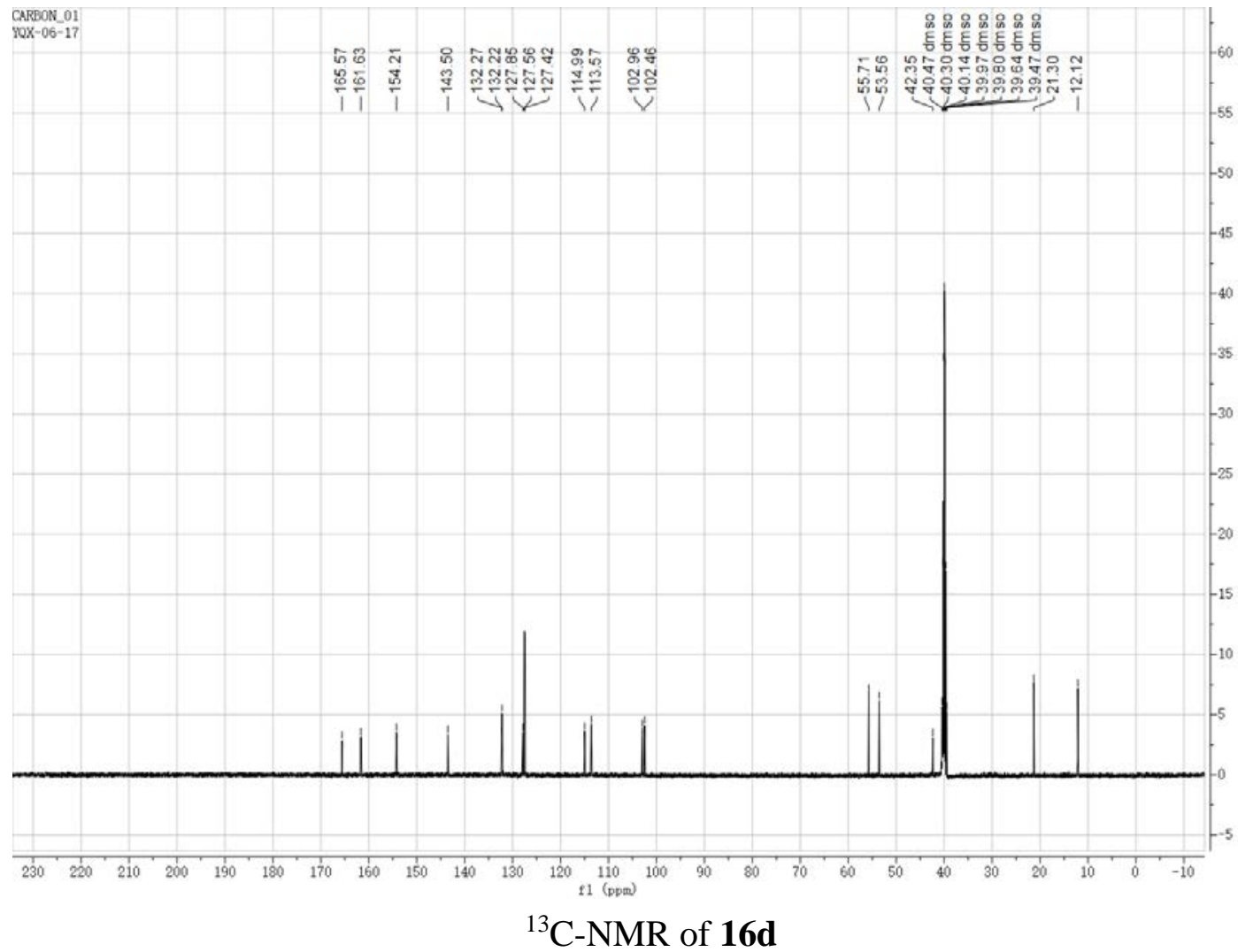



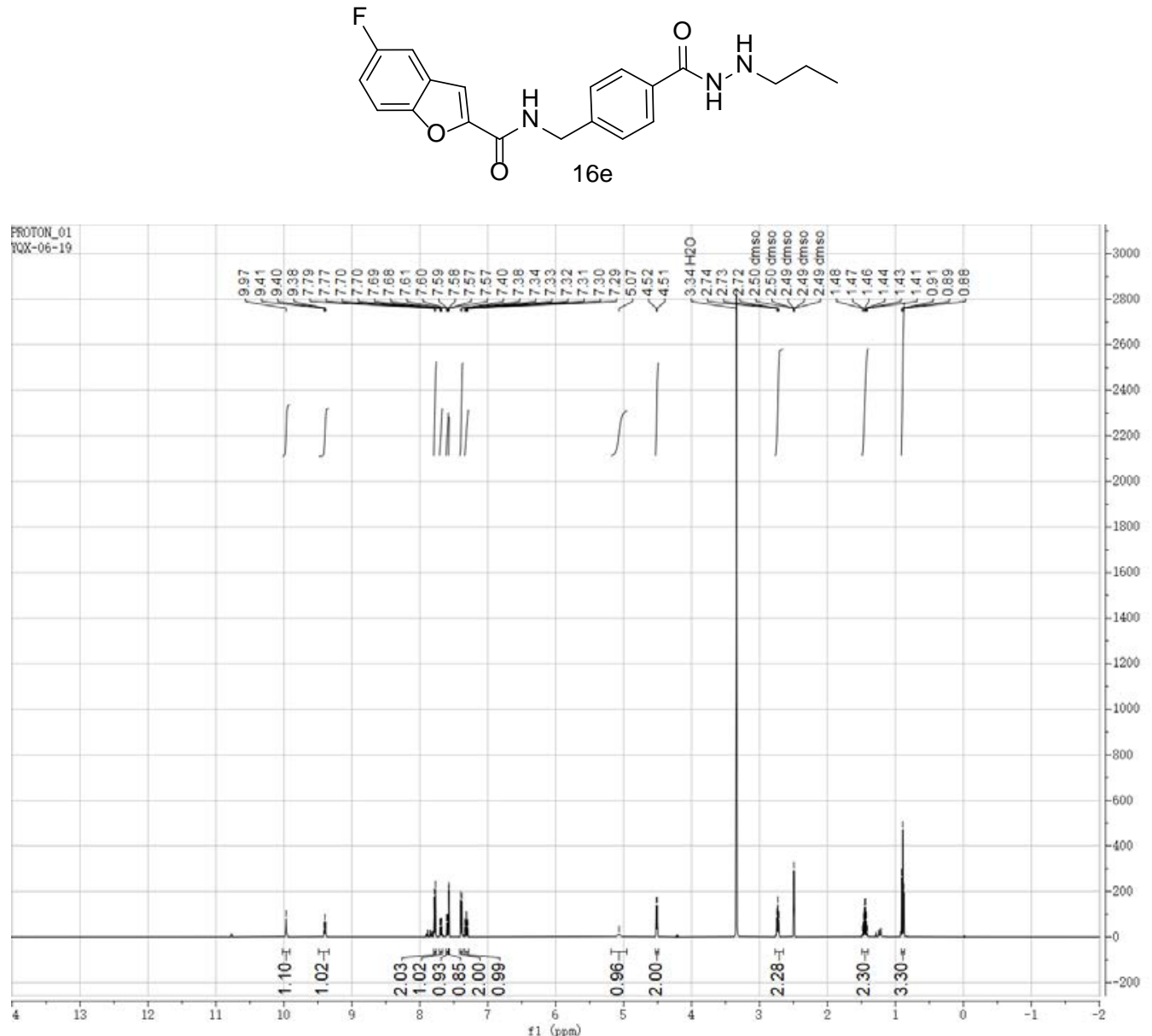

${ }^{1} \mathrm{H}-\mathrm{NMR}$ of $\mathbf{1 6 e}$

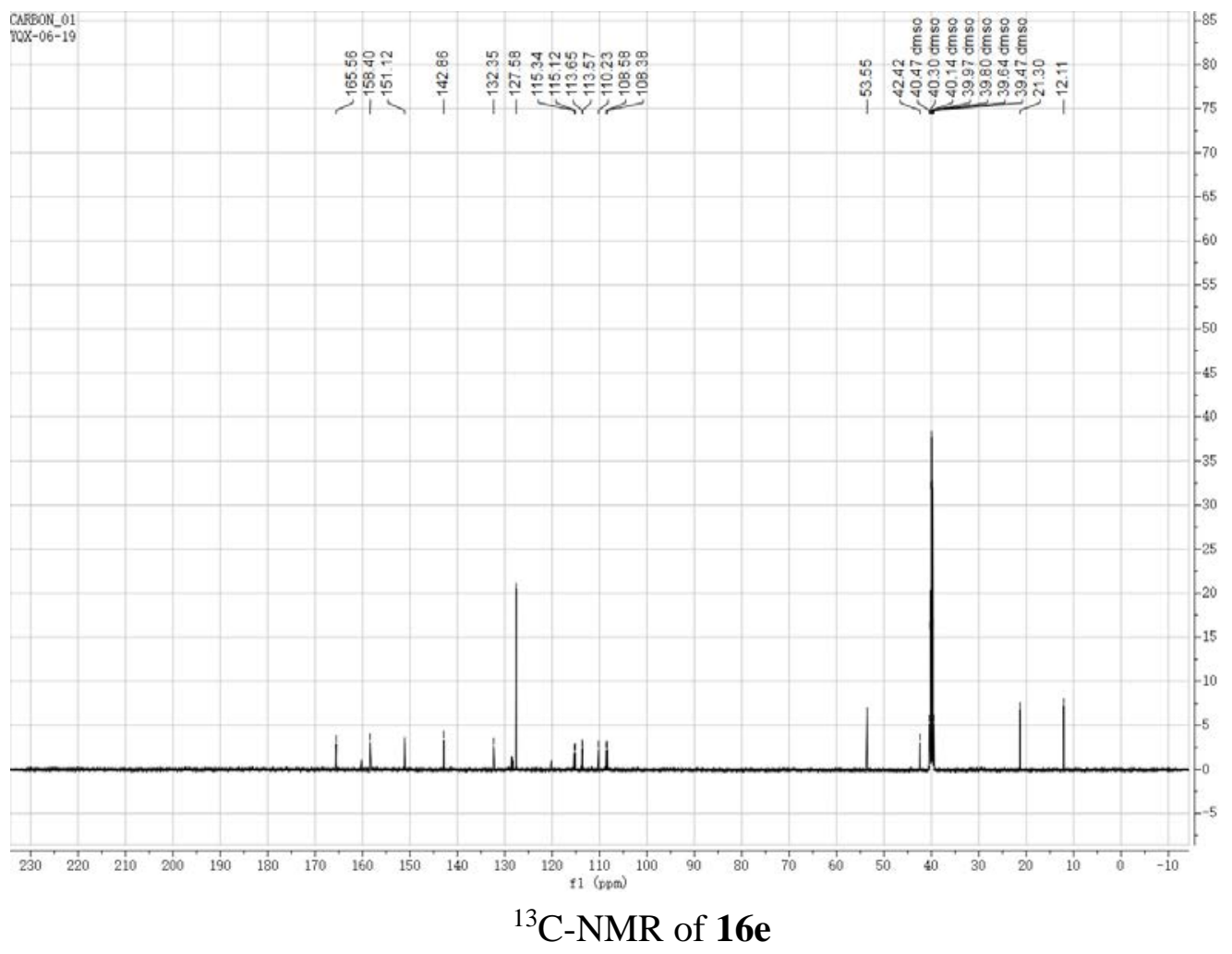



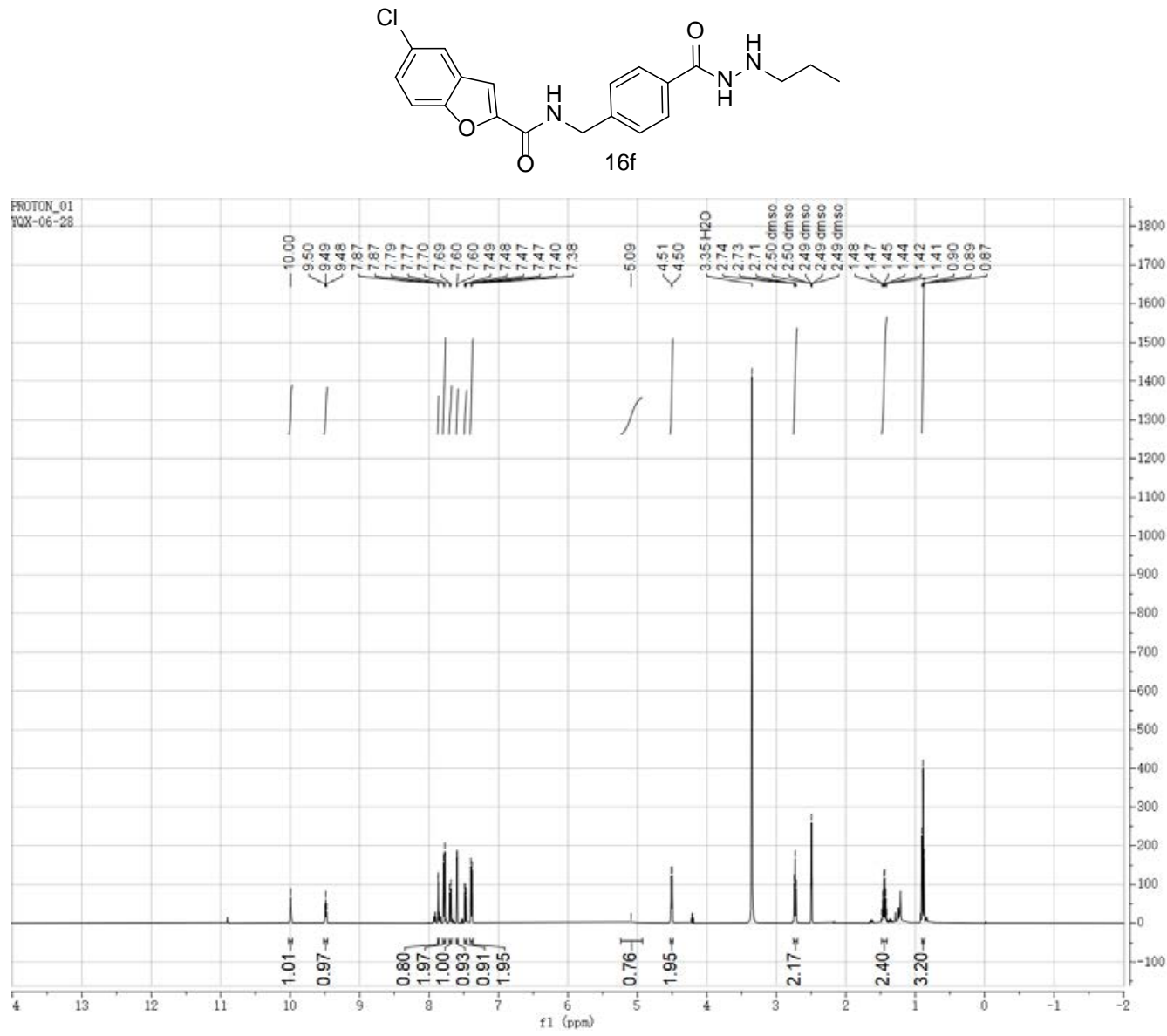

${ }^{1} \mathrm{H}-\mathrm{NMR}$ of $\mathbf{1 6 f}$

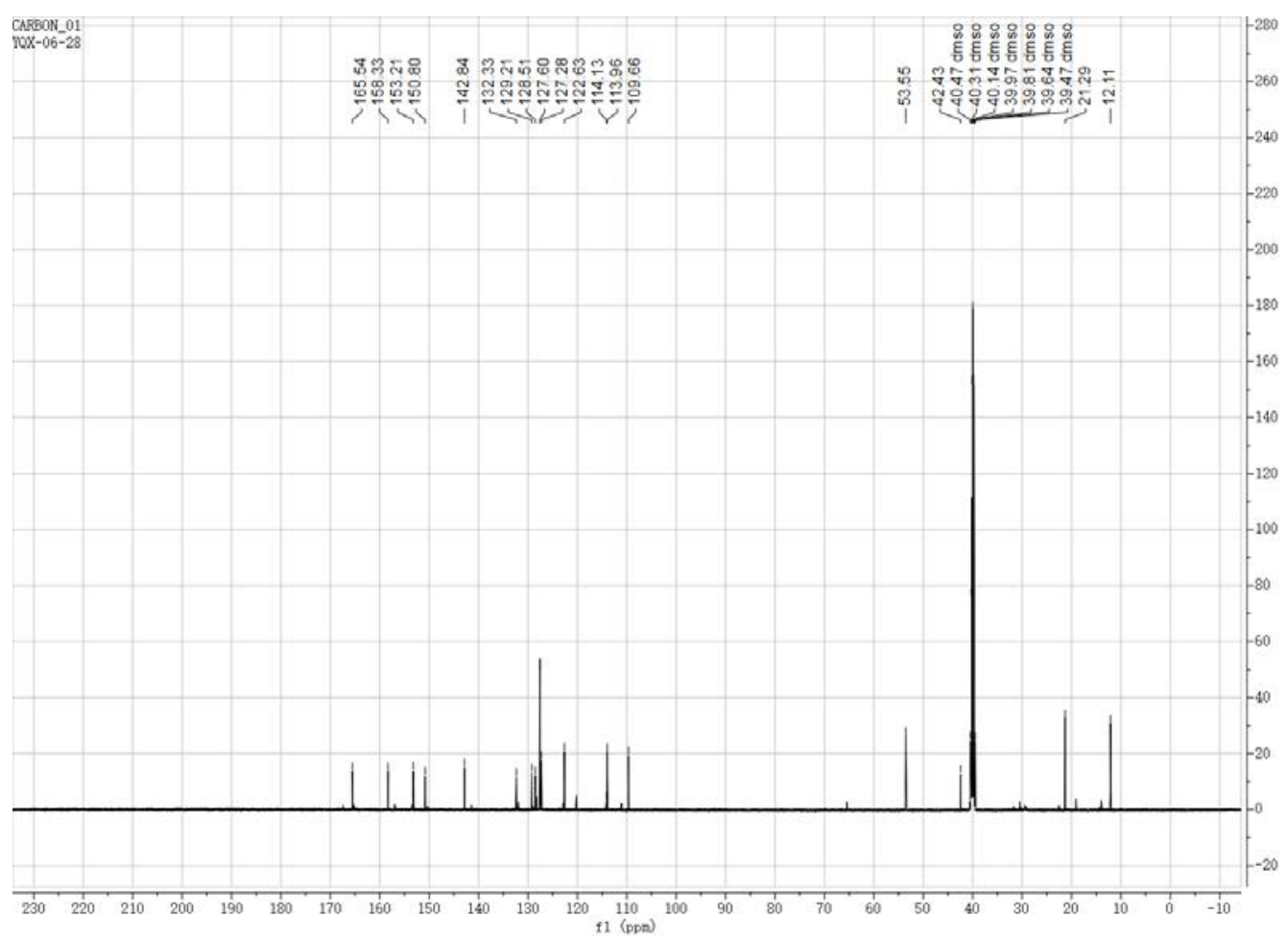

${ }^{13} \mathrm{C}-\mathrm{NMR}$ of $\mathbf{1 6 f}$ 

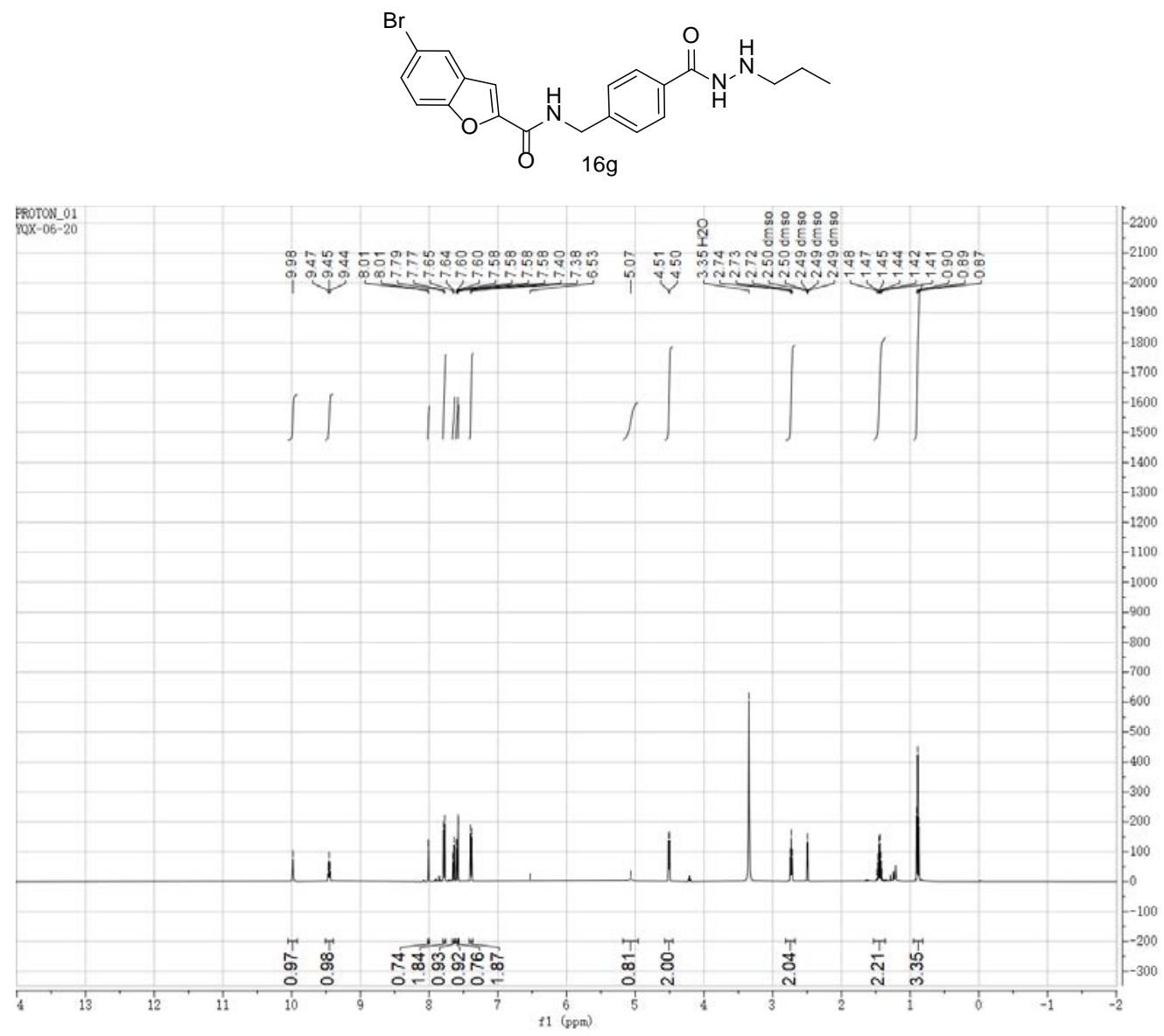

${ }^{1} \mathrm{H}-\mathrm{NMR}$ of $\mathbf{1 6 g}$

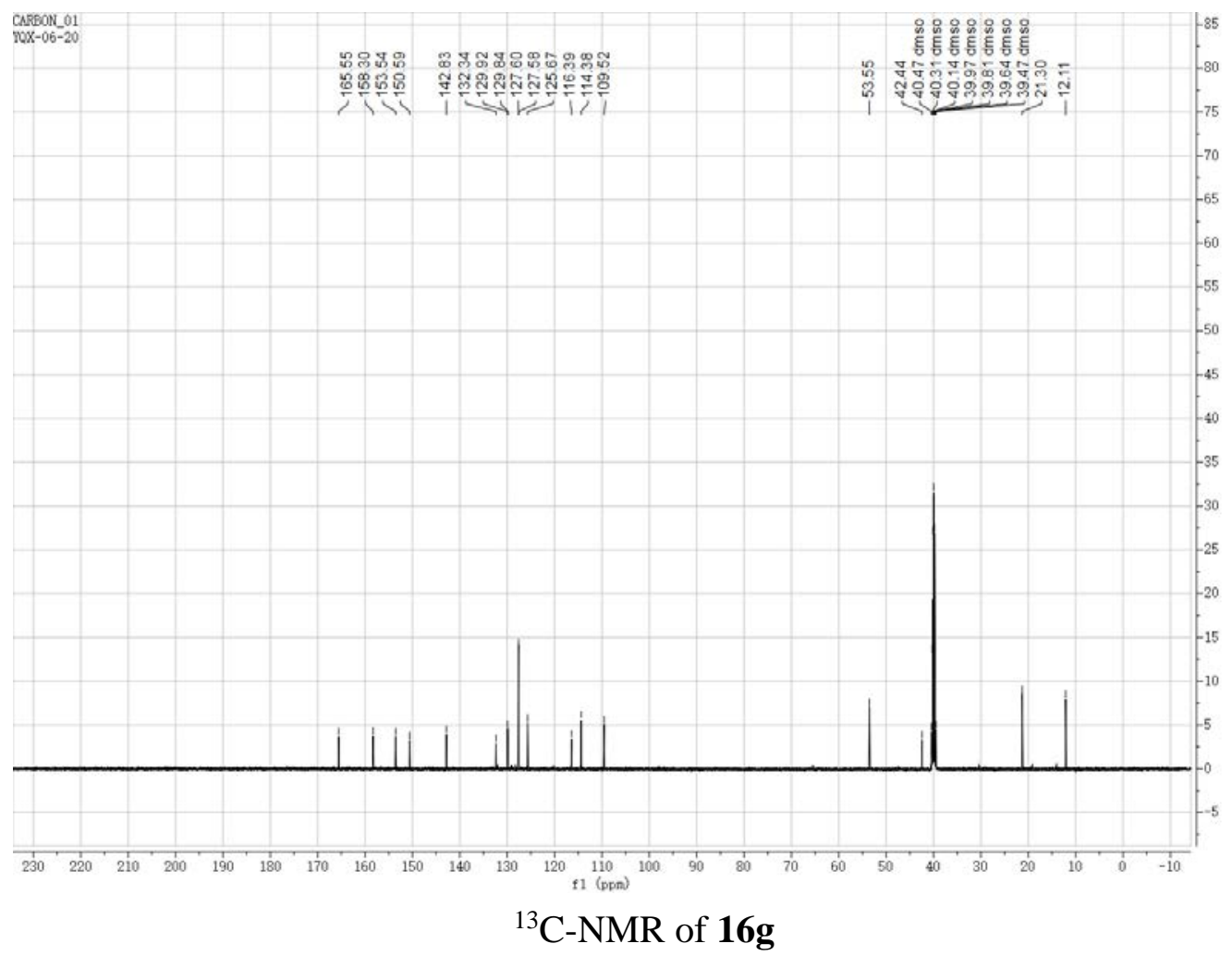



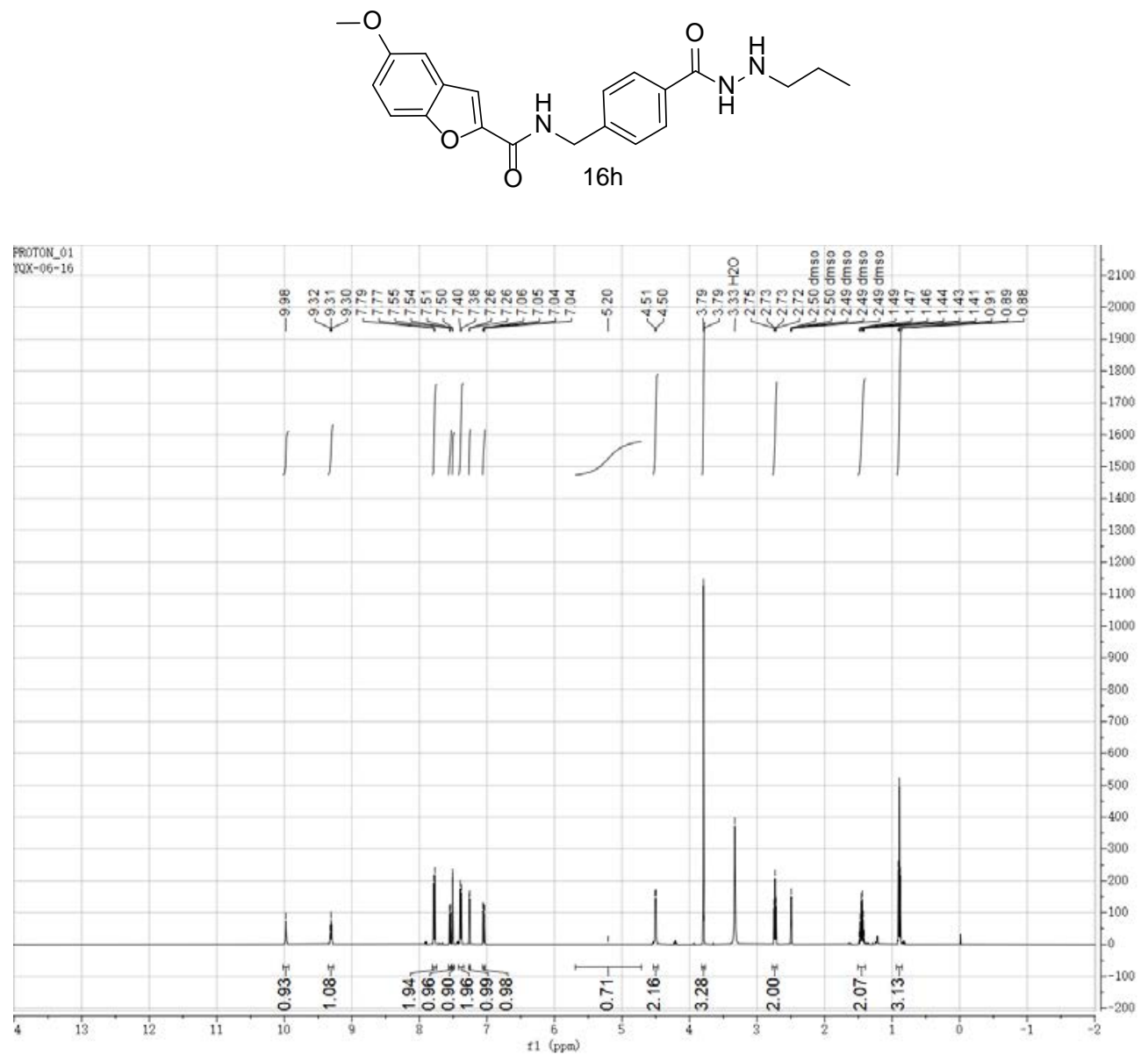

${ }^{1} \mathrm{H}-\mathrm{NMR}$ of $\mathbf{1 6 h}$

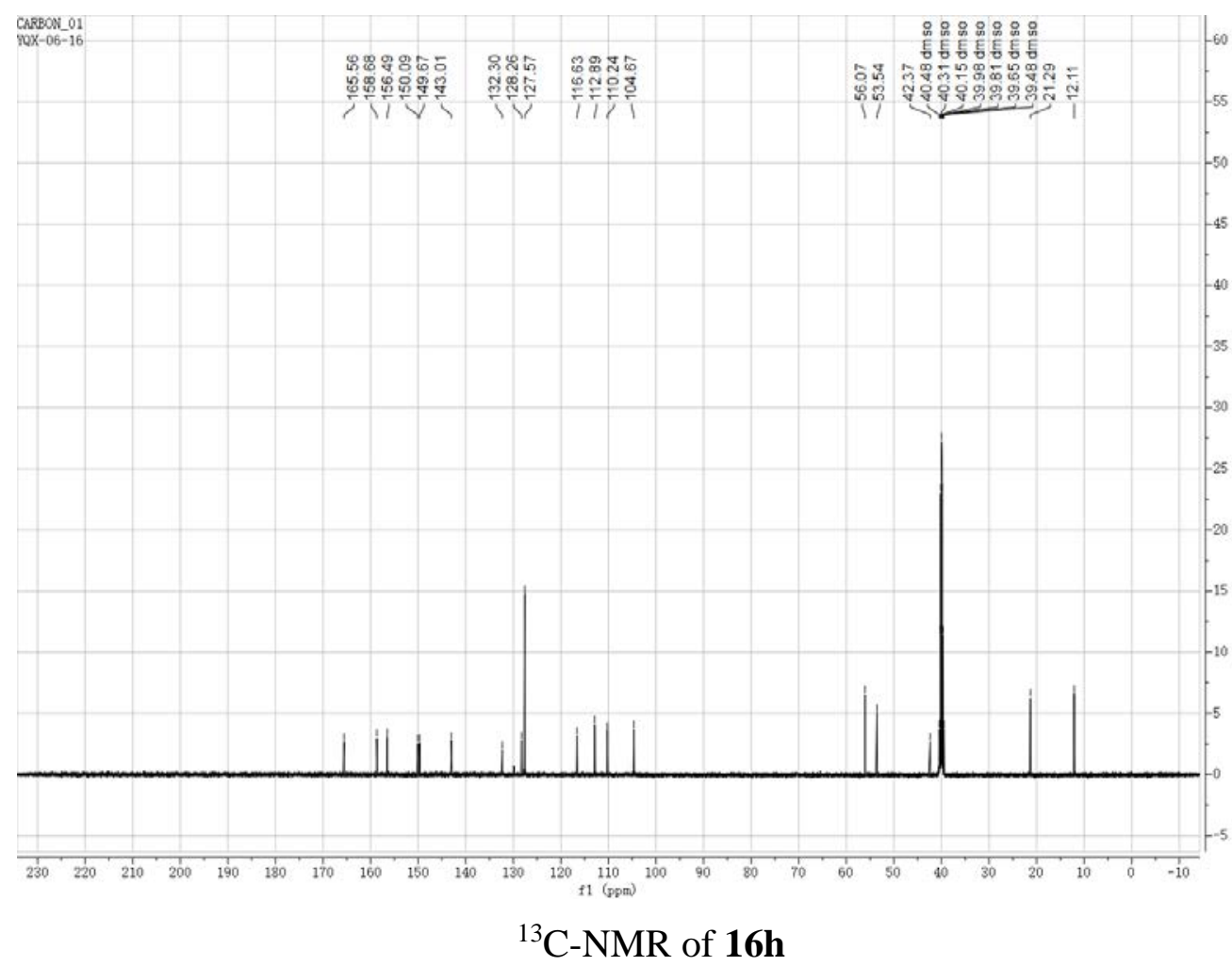




\section{HPLC purity of all the products}

All target compounds were at least 95\% pure as confirmed via UV detection of HPLC, performed on an Agilent 1260 Infinity II HPLC instrument using an Agilent 5 TC-C18(2) C18 column (5 $\mu$ m, 4.6 $\mathrm{mm} \times 250 \mathrm{~mm}$ ) with a gradient of water/acetonitrile=3:2.

1. 11a purity: $99.59 \%$

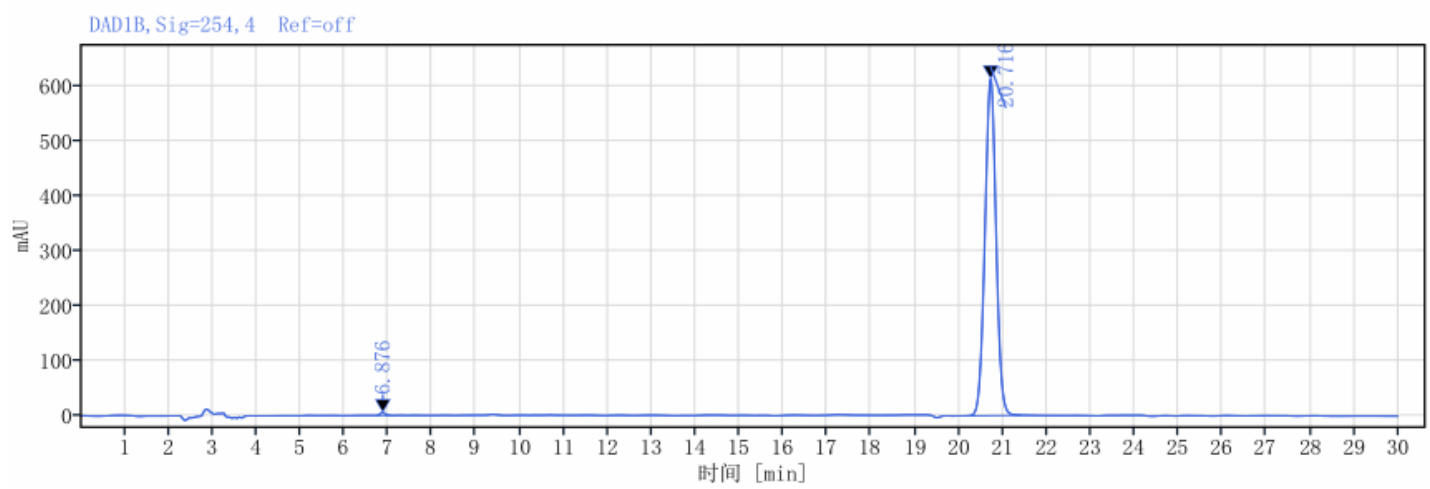

2. 11b purity: $98.77 \%$

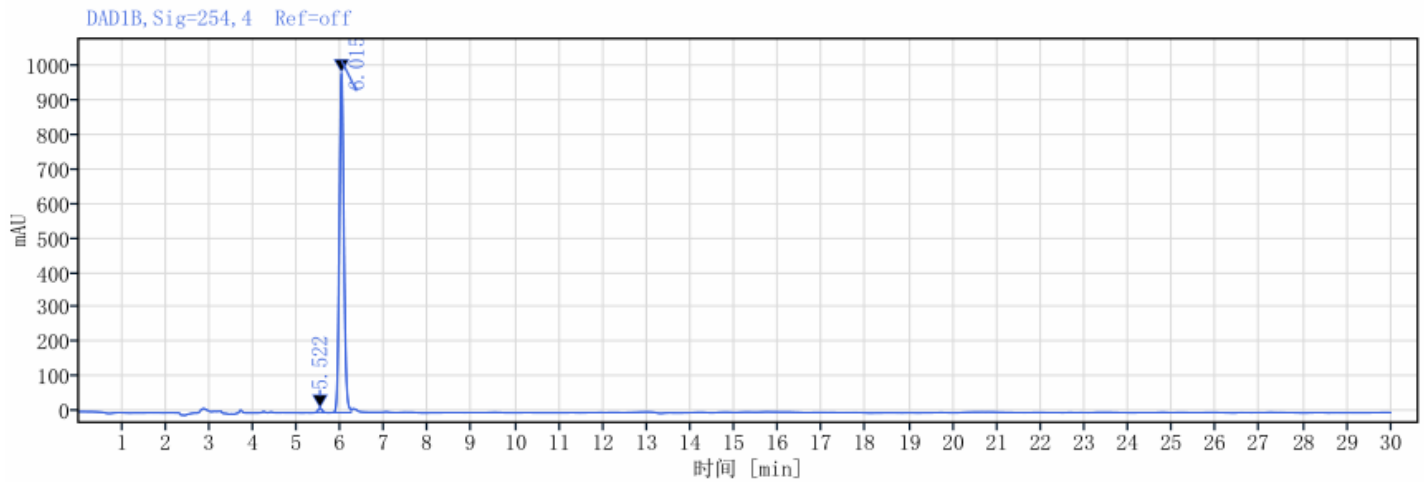

3. 11c purity: $100 \%$

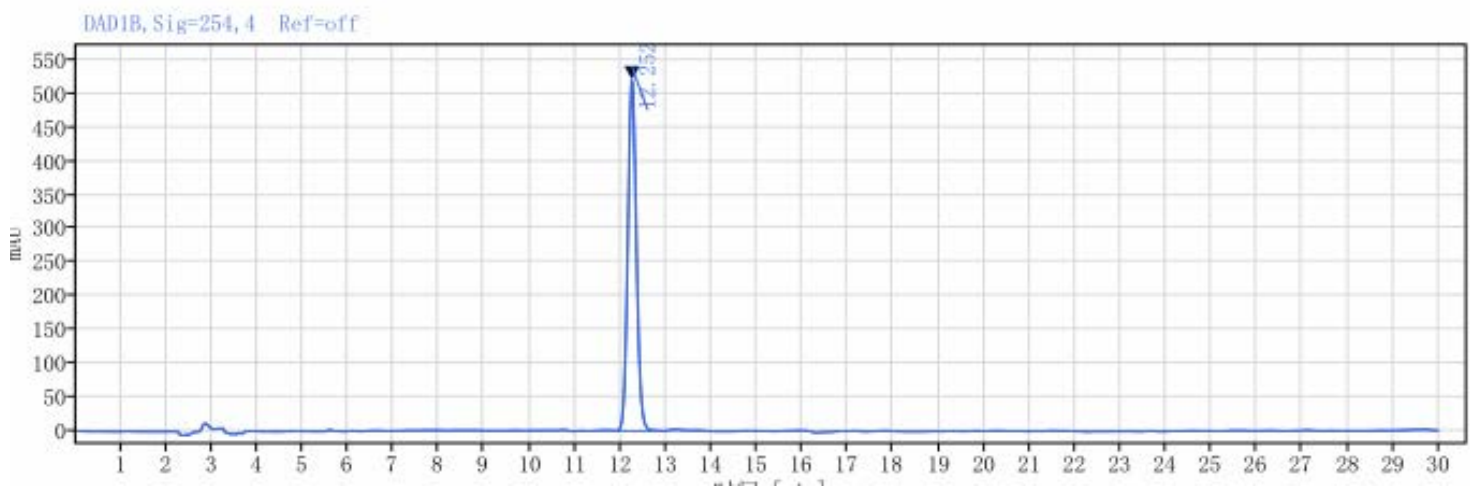


4. 11d purity: $98.45 \%$

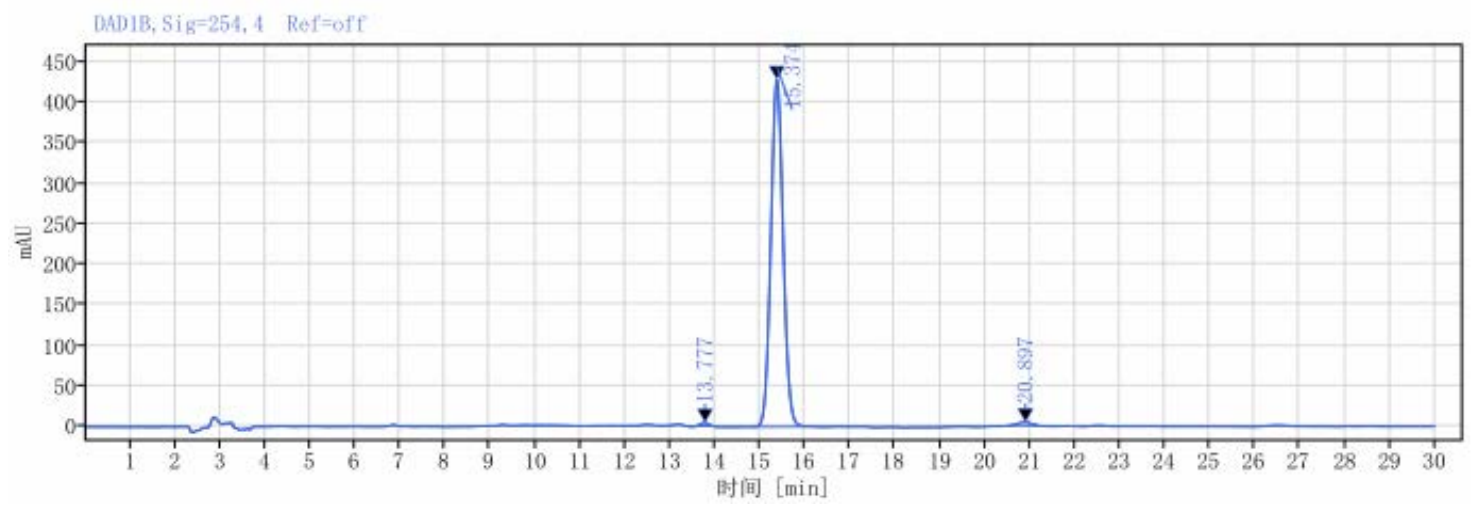

5. 11e purity $99.00 \%$

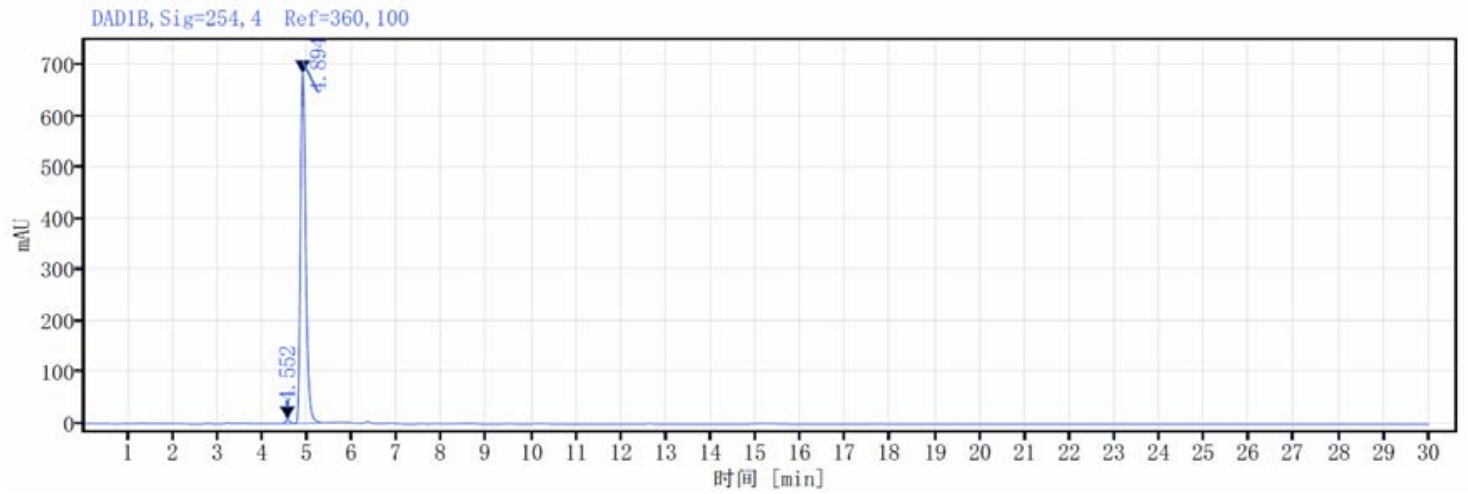

6. 11f purity: $99.13 \%$

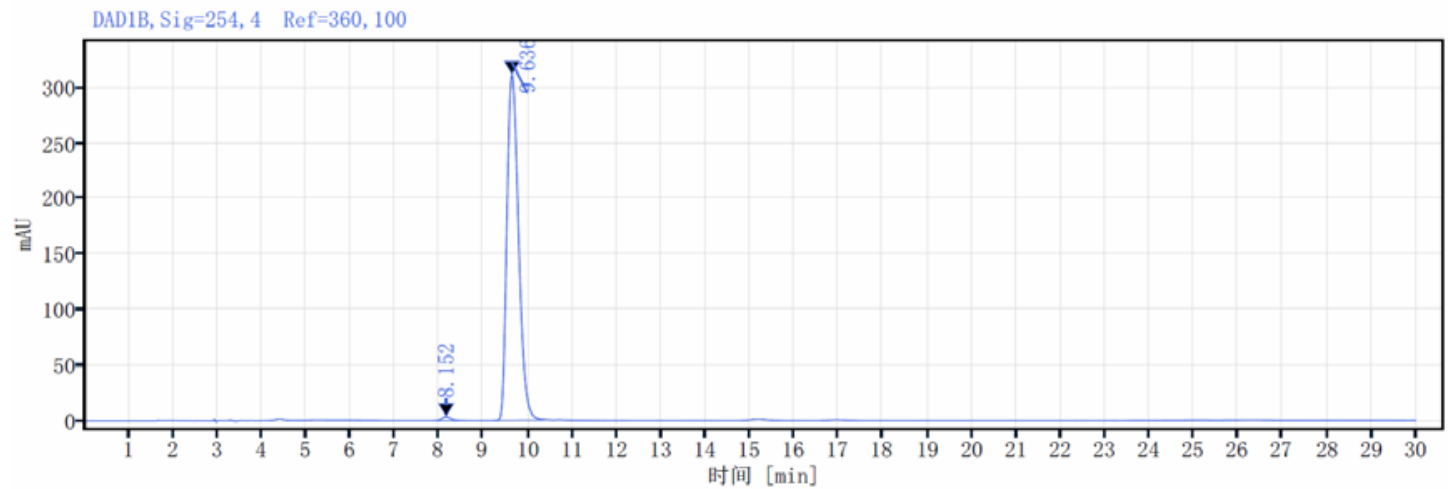

7. 11g purity: $99.71 \%$

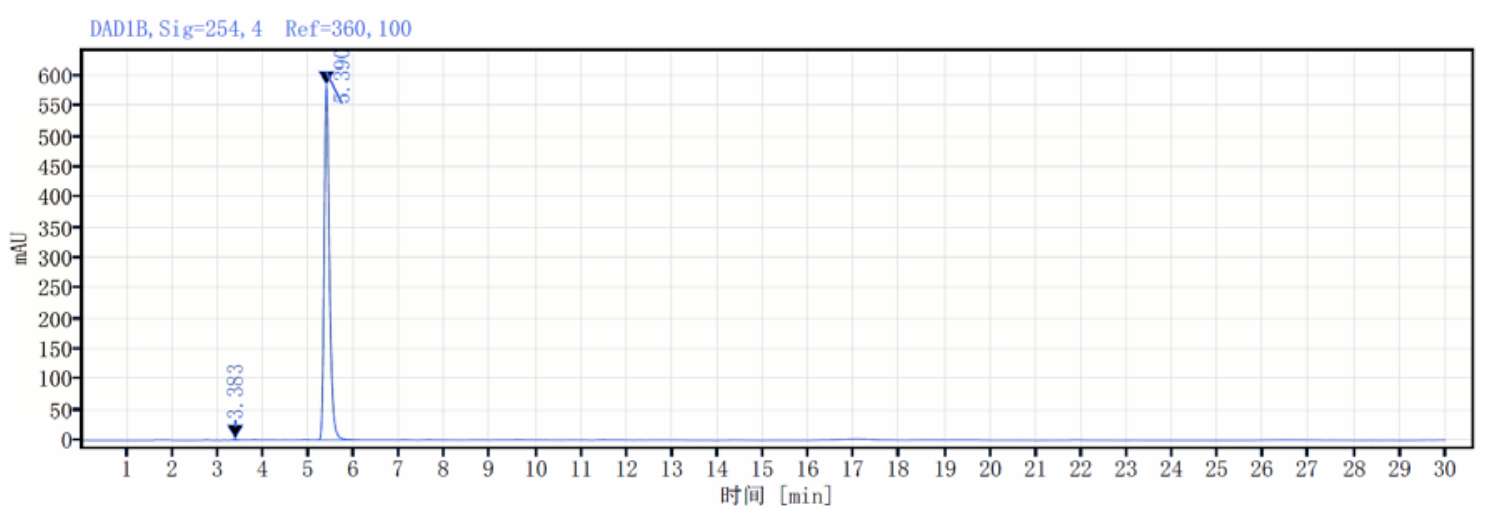


8. 11h purity $99.07 \%$

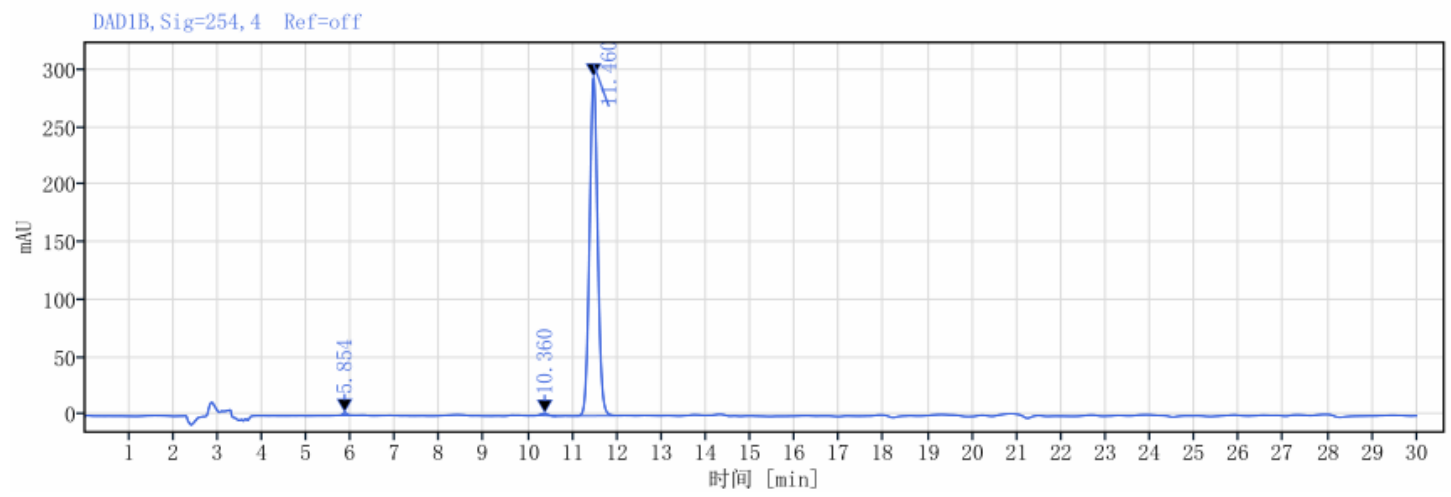

9. 11 i purity $99.79 \%$

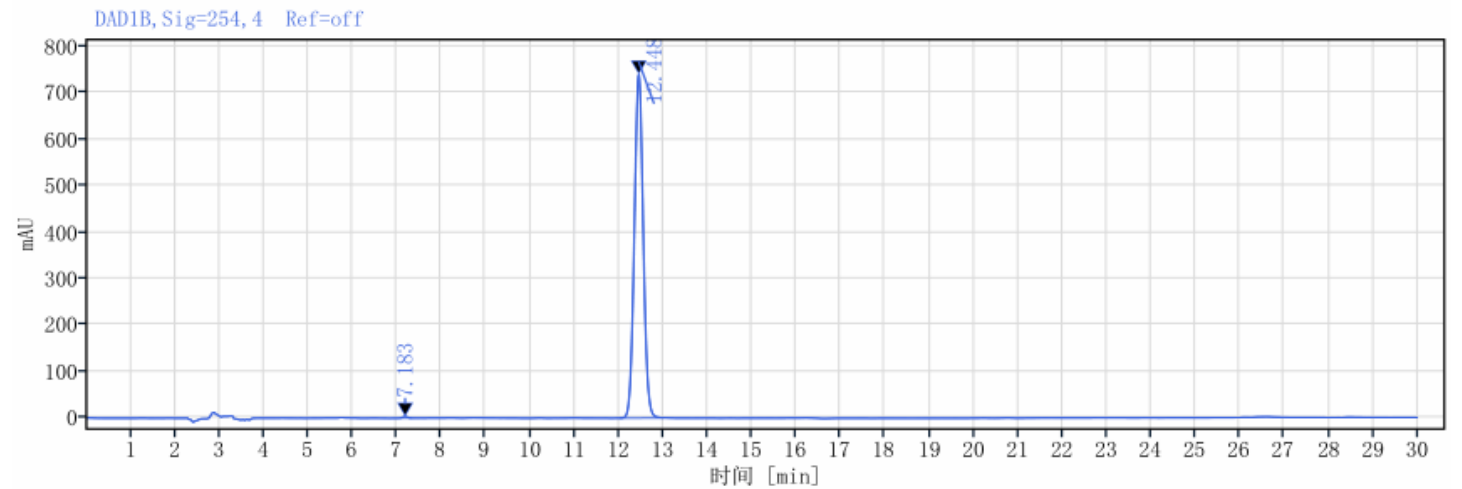

10. 11j purity $100 \%$

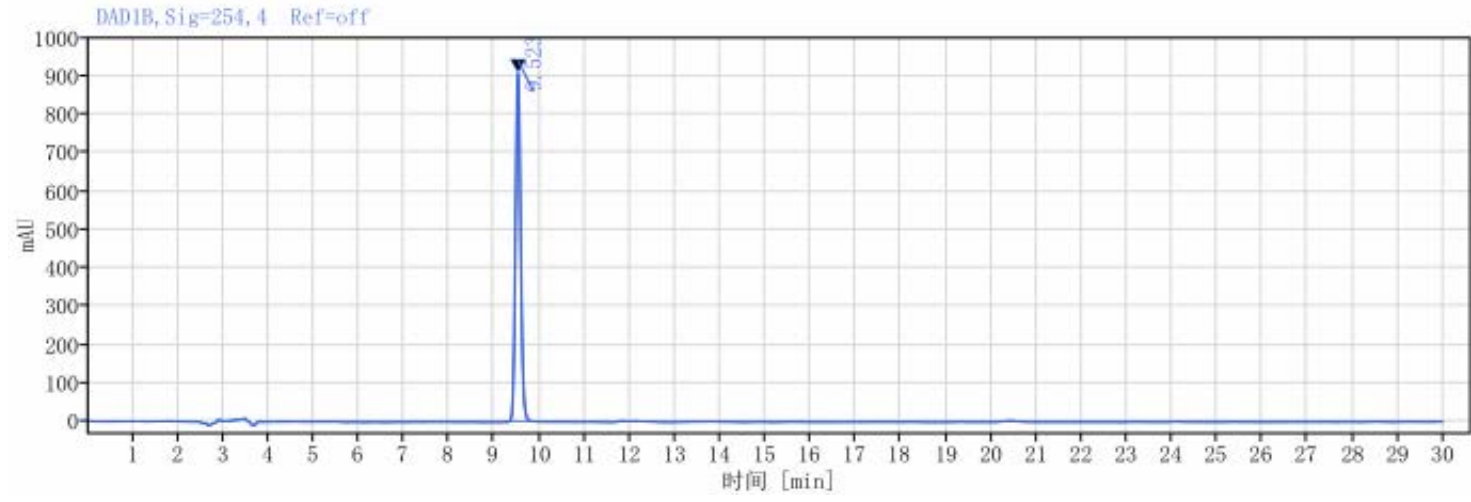

11. 11k purity $100 \%$

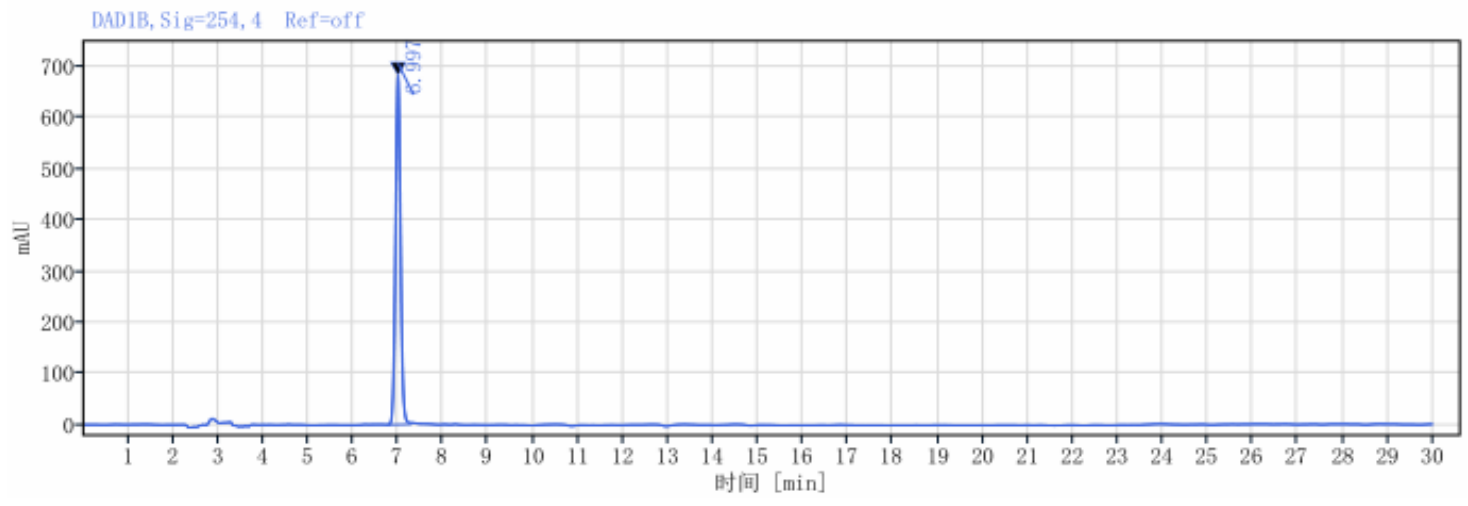


12. 11l purity: $98.26 \%$

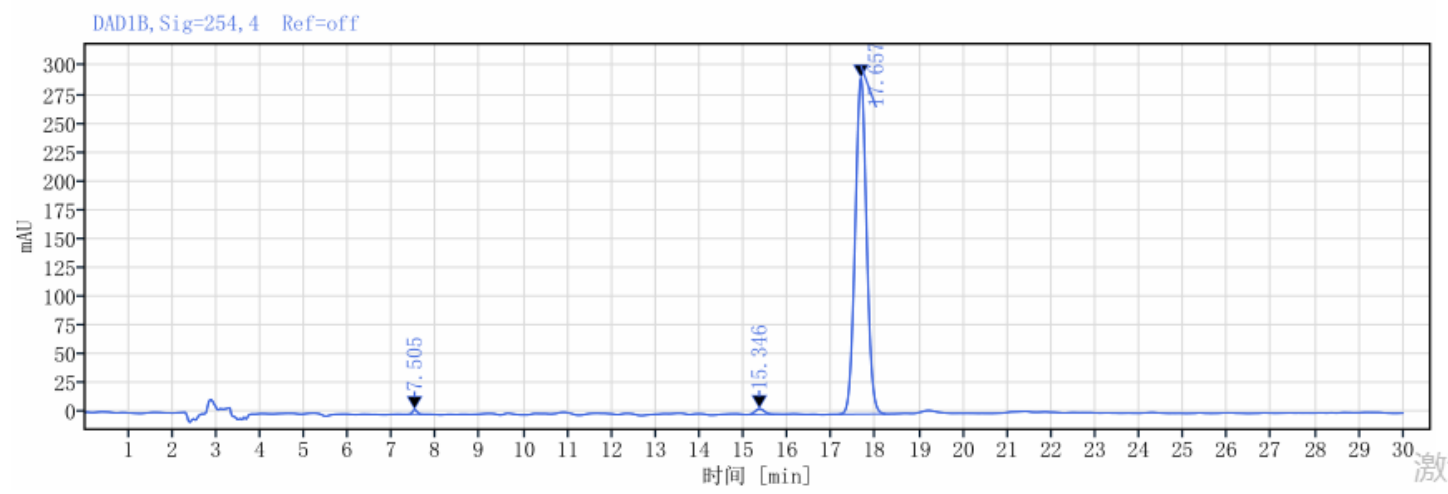

13. 11m purity $96.60 \%$

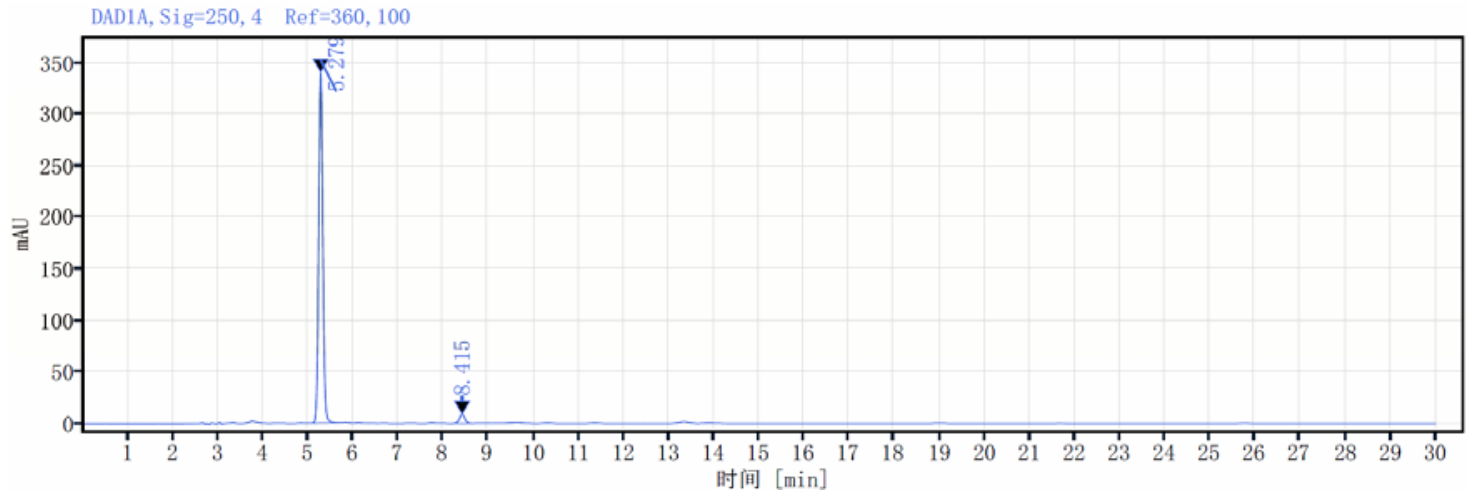

14. 11n purity $100 \%$

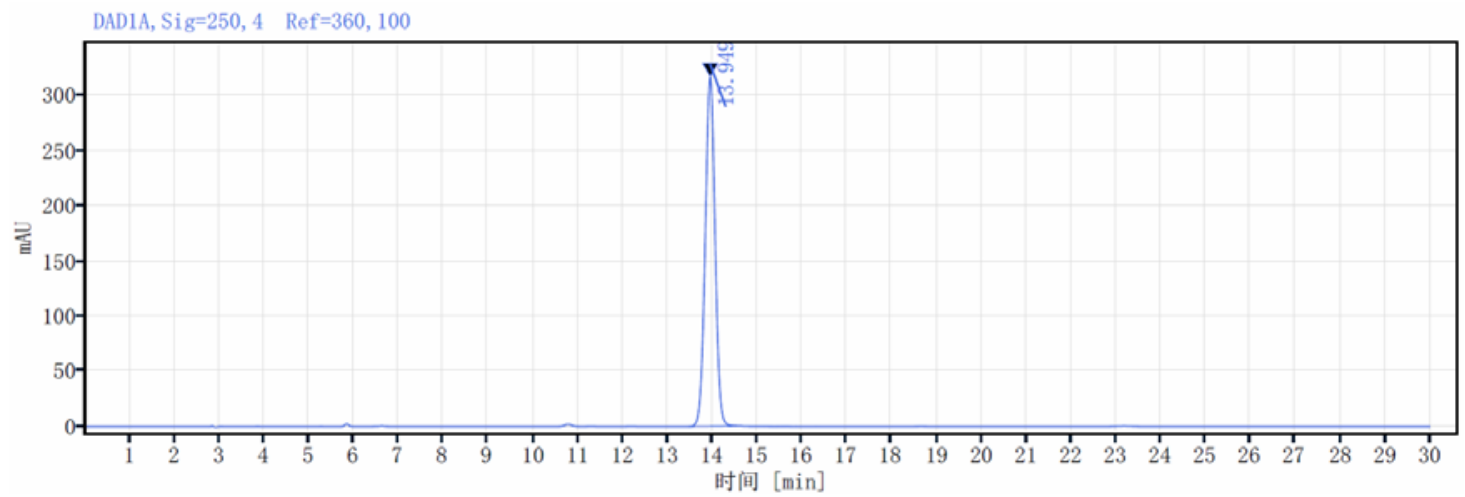

15. 16a purity: $100 \%$

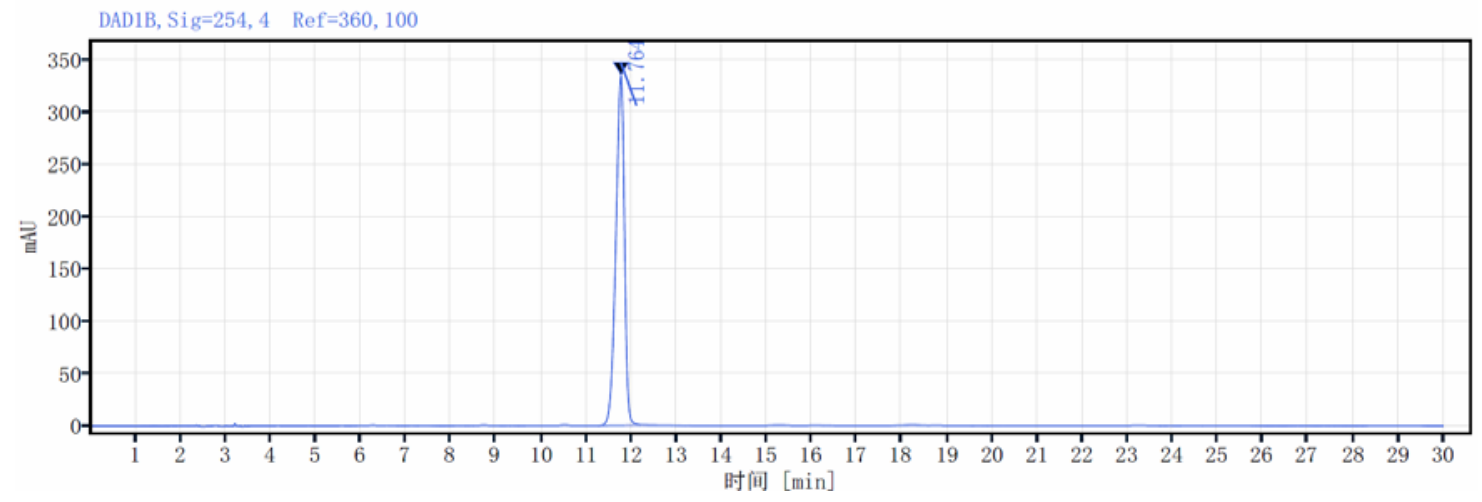


16. 16b purity $100 \%$

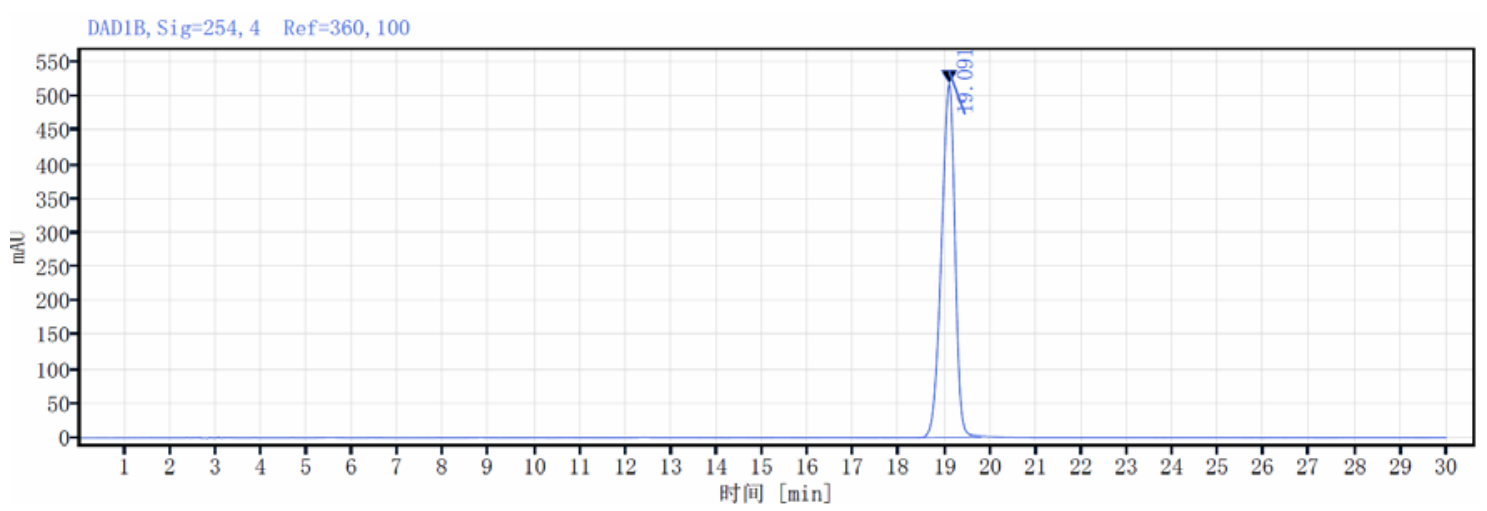

17. 16c purity $96.00 \%$

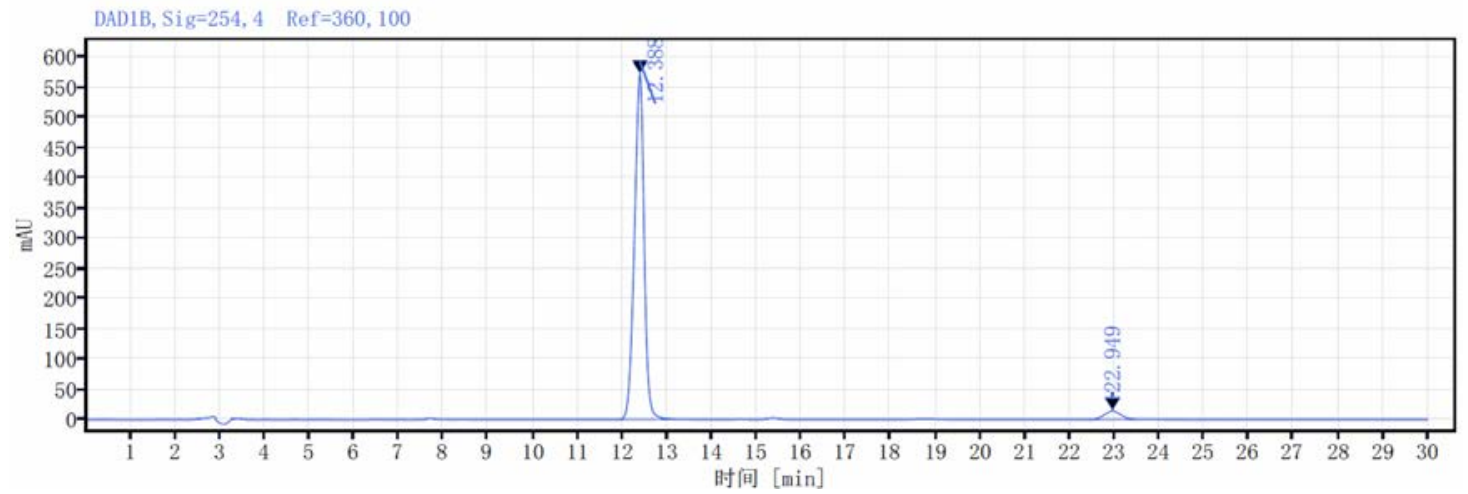

18. 16d purity $100 \%$

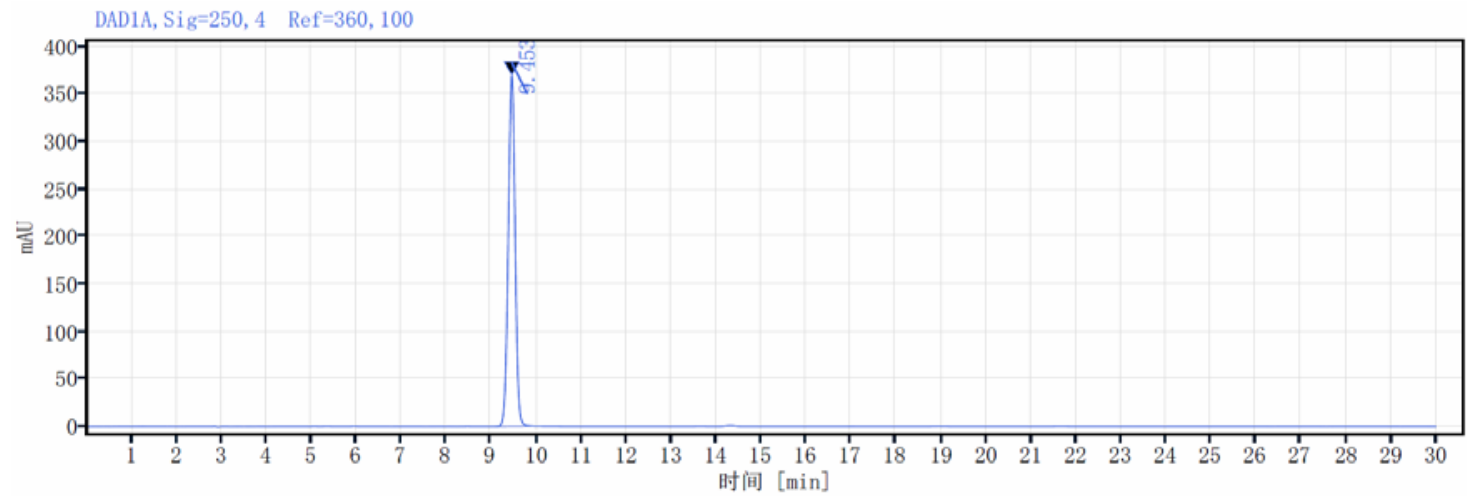

19. 16e purity $98.74 \%$

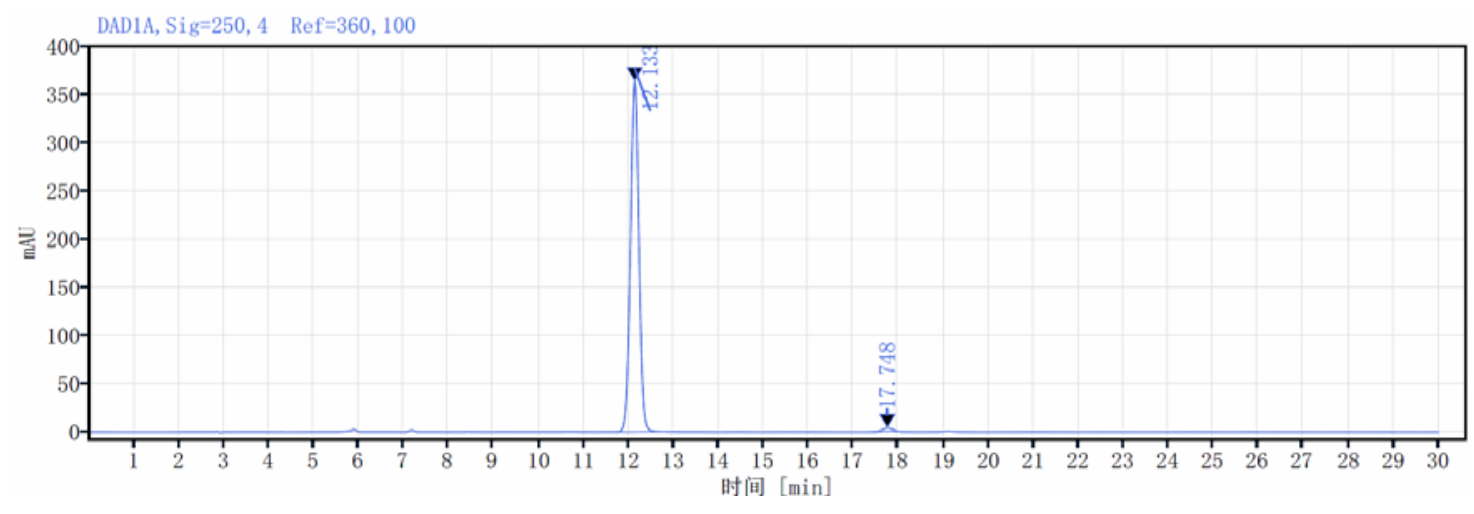


20. 16f purity $96.35 \%$

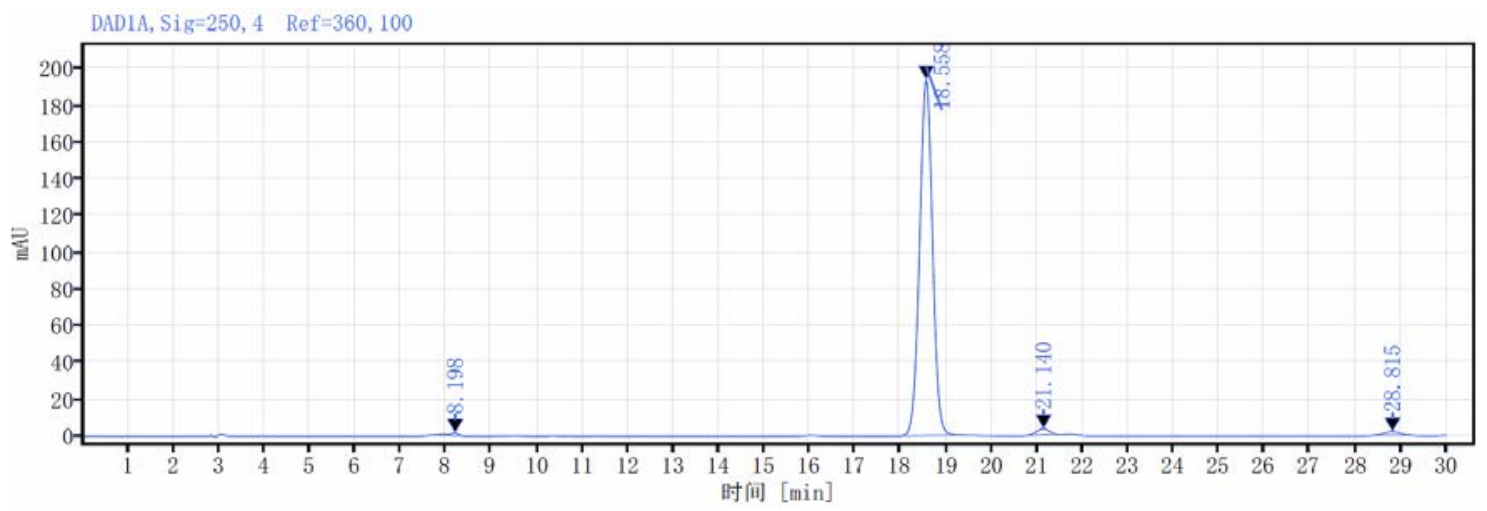

21. 16g purity $99.79 \%$

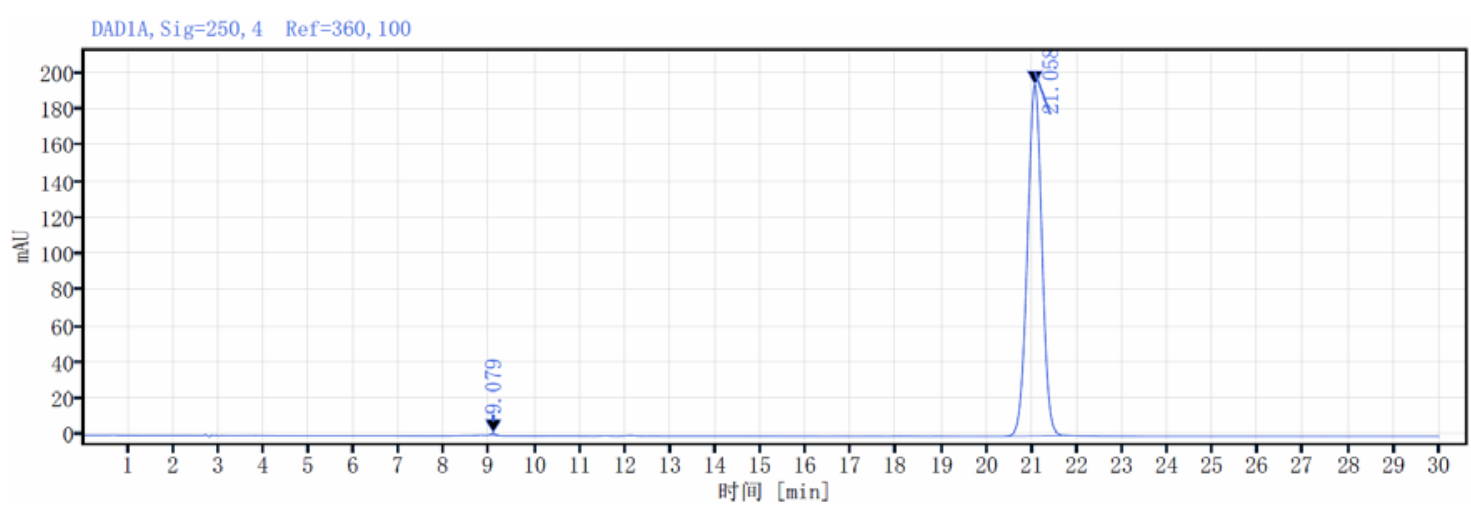

22. 16h purity $98.15 \%$

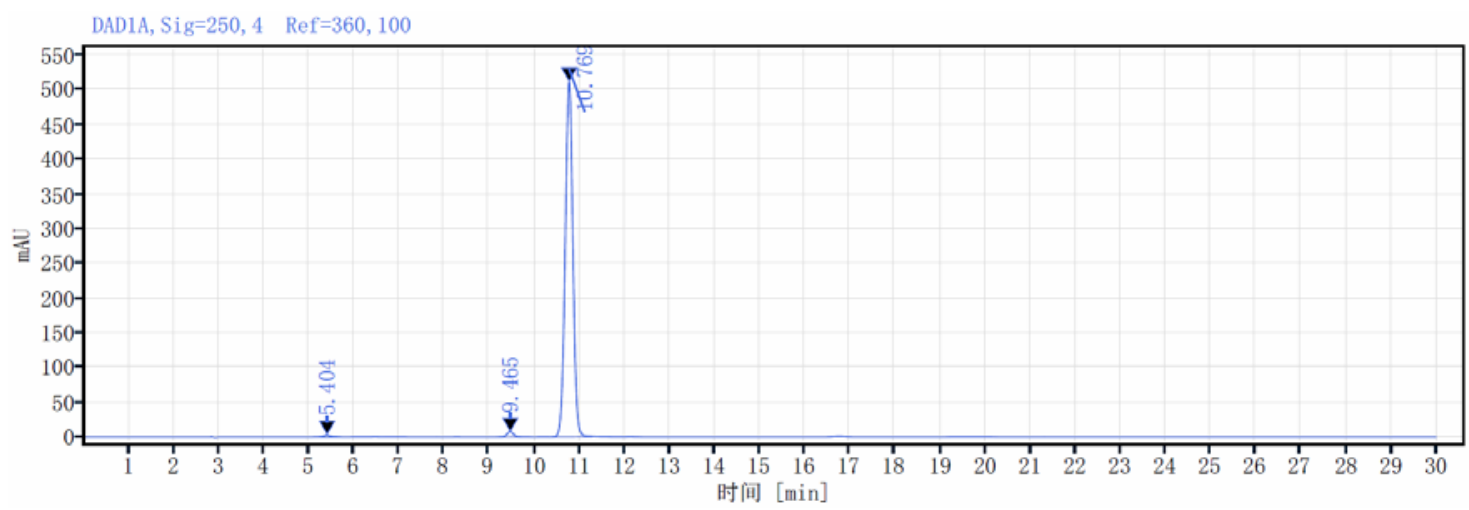

